\title{
Examining factors associated with faking-good responding on the Child Abuse Potential Inventory
}

Amanda H. Costello

West Virginia University

Follow this and additional works at: https://researchrepository.wvu.edu/etd

\section{Recommended Citation}

Costello, Amanda H., "Examining factors associated with faking-good responding on the Child Abuse Potential Inventory" (2014). Graduate Theses, Dissertations, and Problem Reports. 619.

https://researchrepository.wvu.edu/etd/619

This Dissertation is protected by copyright and/or related rights. It has been brought to you by the The Research Repository @ WVU with permission from the rights-holder(s). You are free to use this Dissertation in any way that is permitted by the copyright and related rights legislation that applies to your use. For other uses you must obtain permission from the rights-holder(s) directly, unless additional rights are indicated by a Creative Commons license in the record and/ or on the work itself. This Dissertation has been accepted for inclusion in WVU Graduate Theses, Dissertations, and Problem Reports collection by an authorized administrator of The Research Repository @ WVU.

For more information, please contact researchrepository@mail.wvu.edu. 
Examining factors associated with faking-good responding on the Child Abuse Potential Inventory

Amanda H. Costello

Dissertation submitted to

the Eberly College of Arts and Sciences at West Virginia University

in partial fulfillment of the requirements for the degree of

Doctor of Philosophy in Psychology

Cheryl B. McNeil, Ph.D., Chair

William J. Fremouw, Ph.D.

Natalie J. Shook, Ph.D.

Steven G. Kinsey, Ph.D.

Leslie E. Tower, Ph.D.

Department of Psychology

Morgantown, West Virginia

2014

Keywords: Child maltreatment; parenting capacity assessments; faking-good responding

Copyright 2014 Amanda H. Costello 


\section{ABSTRACT \\ Examining factors associated with faking-good responding on the Child Abuse Potential Inventory Amanda H. Costello}

Child maltreatment (e.g., physical abuse, sexual abuse, emotional abuse, and neglect) remains a serious public health issue which affects an estimated $19 \%$ of victims in the United States (Fang, Brown, Florence, \& Mercy, 2013; U.S. Department of Health and Human Services, 2010), and therefore, it remains important to continue to engage in quality control of the assessment, prevention, and treatment services for parents and children who have been involved in child maltreatment. Parenting capacity assessments (PCAs) are typically ordered in these cases to offer diagnostic impressions of and treatment recommendations for the referred parent (Budd, Connell, \& Clark, 2011). The Child Abuse Potential Inventory (CAPI; Milner, 1986) is a measure that is widely used in PCAs. Faking good on the CAPI during PCAs has been identified as a behavioral pattern that is often observed, thus invalidating important information derived from these assessments. However, despite the negative consequences that typically come from faking-good profiles (i.e., impression of the faking parent as being a liar or manipulative; discarding data from a faking parent in evaluations), few studies have been published which have sought to directly probe for these characteristics. The current study utilized a prospective, "real world" design, in which participants who were receiving parenting services either at community mental health centers or due to involvement with Child Protective Services, were recruited. Sixty-two parents (30 parents who were considered "treatment-seeking without child protection" and 32 parents with child protective services involvement) completed study procedures. In this sample, 22 (35.5\%) parents had an invalid profile on the CAPI due to an elevated Faking-good Index. Faking and non-faking parents were compared across four major domains: treatment group (e.g., involvement in child protective services or not), cognitive functioning, measured by scores on the Wechsler Abbreviated Scale of Intelligence, Second Edition (WASI-II; Wechsler, 2011) and the reading comprehension subscale of the Wechsler Individual Achievement Test, Third Edition (WIAT-III; Wechsler, 2009), self-reported psychopathy, measured by the Levenson Self-Report Psychopathy Scale (LSRP; Levenson, Kiehl, \& Fitzpatrick, 1995), and a positivity bias (i.e., the tendency to selectively attend to positive over negative information in the environment, even when it is unrealistic) measured by scores from the BeanFest paradigm (Fazio, Eiser, \& Shook, 2004). Additionally, faking and non-faking parents were compared across demographic information and other study measures, including the Marlowe Crowne Social Desirability Scale (MCSDS; Crowne \& Marlowe, 1960) and the Beck Depression Inventory, Second Edition (BDI-II; Beck, Steer, \& Brown, 1996). Results from this study supported that faking-good parents had lower intelligence and reading comprehension scores, as well as a positivity bias on the BeanFest. Interestingly, treatment setting (e.g., involvement in child protective services) and psychopathy characteristics did not significantly differentiate faking and non-faking groups. Exploratory analyses revealed a strong association between the CAPI Lie Scale and the Marlowe Crowne Social Desirability Scale (Crowne \& Marlowe, 1960), which provides recent data to suggest these two scales may be measuring a similar construct. Implications of study results, limitations of the study, and future directions for follow-up research will also be discussed. 


\section{Acknowledgements}

First and foremost, I would like to thank Cheryl B. McNeil, Ph.D., for her unwavering support and guidance, as not only the chair of my dissertation, but also my academic and research mentor throughout graduate school at West Virginia University. Words cannot express how much I have learned from Dr. McNeil in the past five years, and how valuable her mentorship has been to me, and I look forward to continuing to work together in the future. I would also like to thank the rest of my dissertation committee members: William J. Fremouw, Ph.D., Natalie J. Shook, Ph.D., Steven G. Kinsey, Ph.D., and Leslie E. Tower, Ph.D. for their support over the last two years. Specifically, I would like to acknowledge Dr. Shook, who provided helpful feedback and support over the last 1.5 years as I learned, used, and interpreted the BeanFest paradigm.

I would also like to thank the wonderful community mental health agencies that aided in recruitment of parents for my study; without their support, this dissertation could not be possible. These agencies are: the Monongalia County Child Advocacy Center (special thank you to Laura Capage, Ph.D.), The WellTree, LLC (Shawn E. Trimble, Executive Director), Fremouw-Sigley Psychological Associates (special thank you to William Fremouw, Ph.D.), Wedgewood Psychiatry Associates (Martin Boone, Ph.D.), Family Behavioral Resources (Uniontown, Pennsylvania office) and the graduate student therapists and faculty supervisors at the Quin Curtis Center at the West Virginia University Department of Psychology. Special thanks go to the fellow graduate student members of Dr. McNeil's lab, who have helped me develop and refine my research over the years. I want to specifically acknowledge Nancy Wallace, B.S., for her tireless work on my dissertation data collection, and without whom this study would be completed. Her professionalism and positive attitude never cease to impress me. Reeva Morton, B.S., was also an integral part of my dissertation, and I want to thank her for her hard work 
during data collection. I would also like to thank Tara Karns, Ph.D for her help with statistical methods on my study, and for her friendship and support throughout graduate school. Finally, I would like to acknowledge Amy D. Herschell, Ph.D., who inspired me to first pursue a Ph.D. in clinical psychology, and whom I am proud to call a mentor for eight years.

I would like to thank my absolutely amazing family: Tom, Ann, and Kyle Costello. You taught me at an early age what it means to work hard, be compassionate to others, and leave a positive mark on the world, and without your love and support, I would not be the person I am today. I would also like to thank Eric Dailey: you have stood by me through ups and downs, distance short and long, and accomplishments and failures. You have taught me what it truly means to love and respect another person, and be loved in return. And finally, I would like to acknowledge my fantastic friends, old and new, near and far. You have kept me smiling, with my head up high, ready to tackle the next challenge or adventure that comes my way. I can only hope to continue to be inspired by the next big and exciting adventures you pursue in your lives. 


\section{Table of Contents}

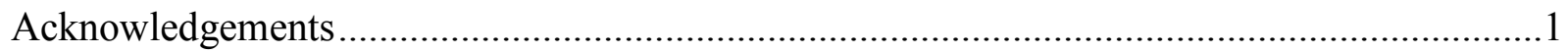

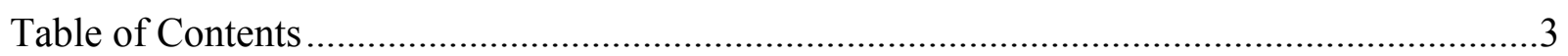

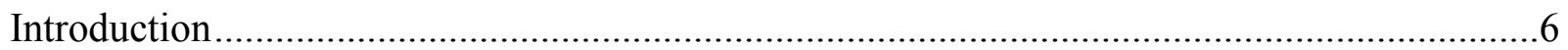

Predicting Child Maltreatment Recidivism ........................................................................6

Use of the Child Abuse Potential Inventory in PCAs..........................................................

Development of the Child Abuse Potential Inventory Lie Scale and Faking-good Index............10

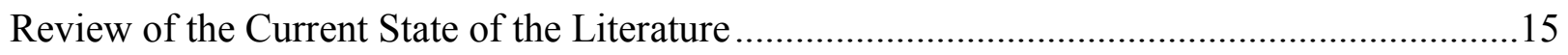

Outcomes of parental faking-good responding .......................................................15

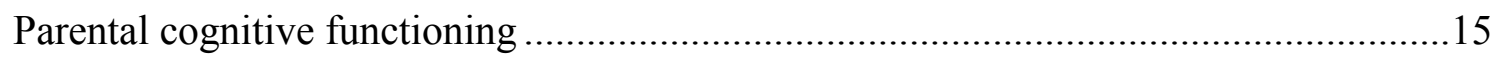

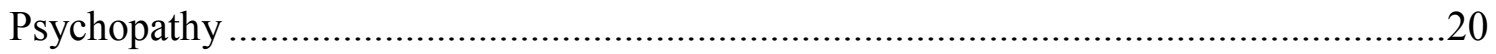

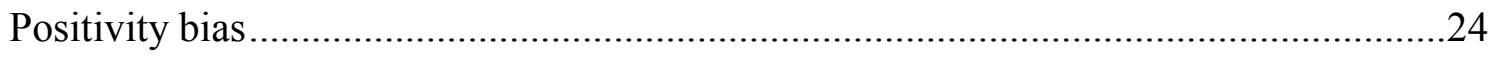

Considerations When Addressing Socially Desirable Responding ...........................................28

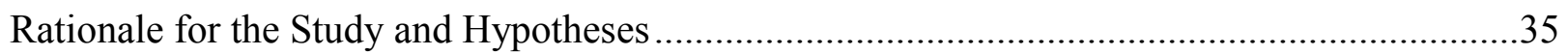

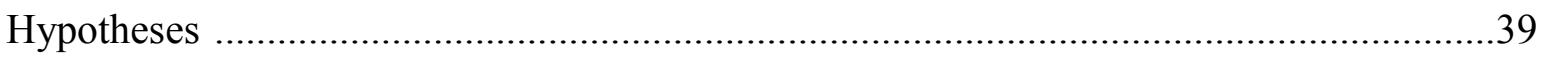

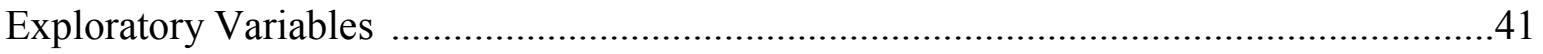

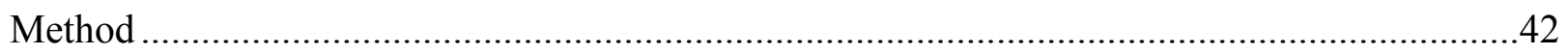

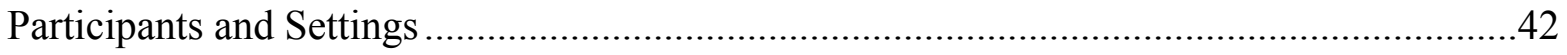

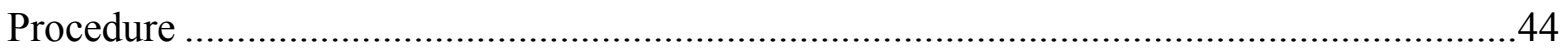

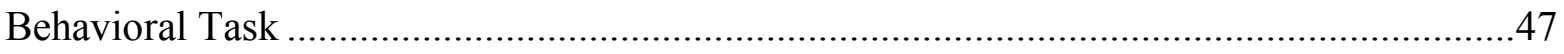

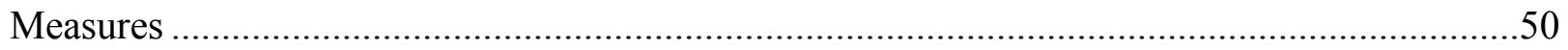

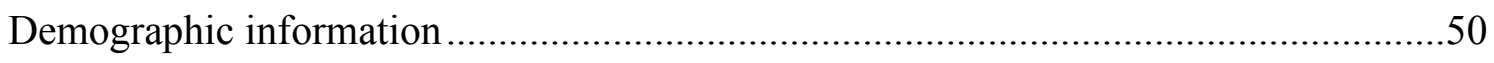

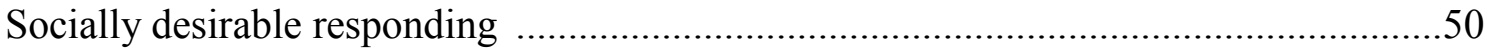




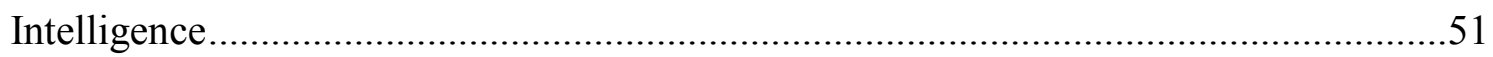

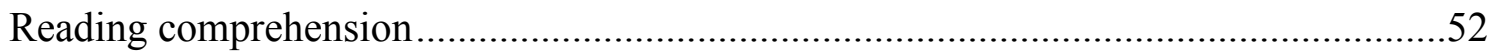

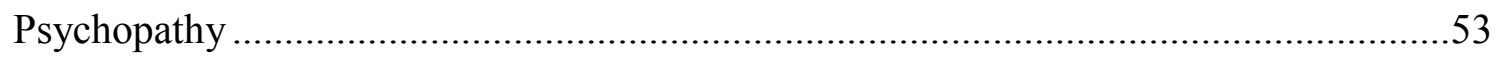

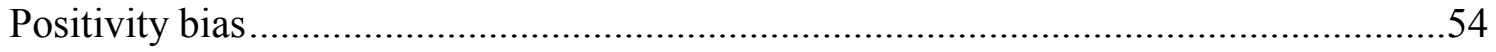

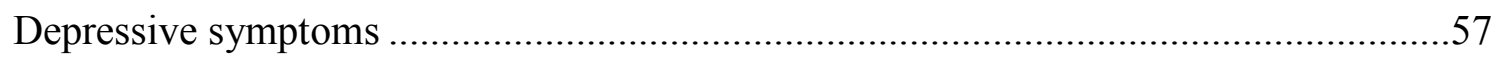

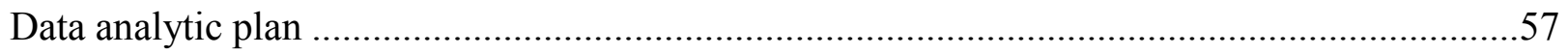

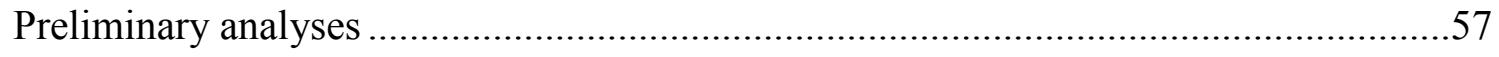

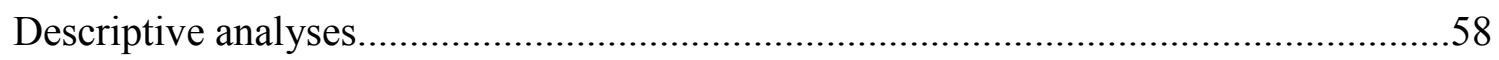

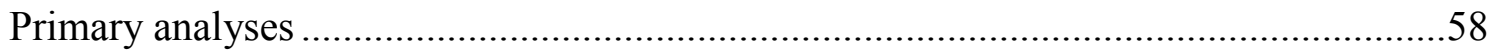

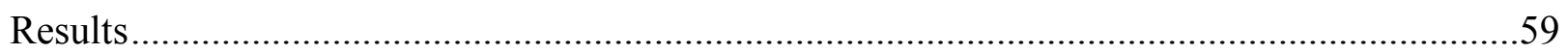

Demographic Characteristics of the Sample................................................................59

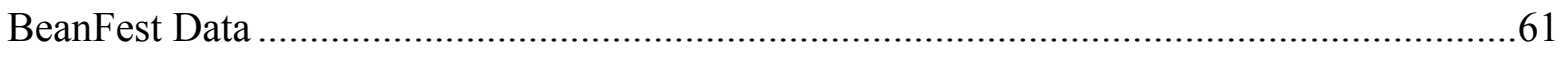

Missing data/response bias analysis ...................................................61

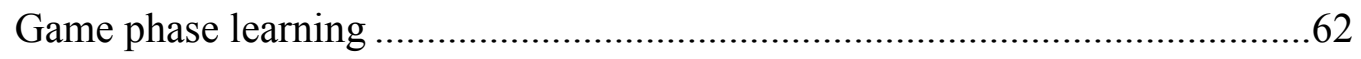

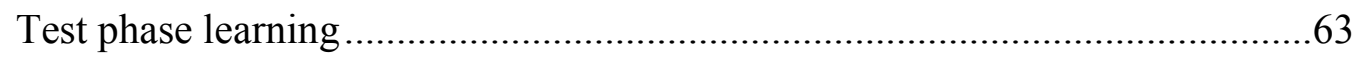

Association of BeanFest variables with demographic information..................65

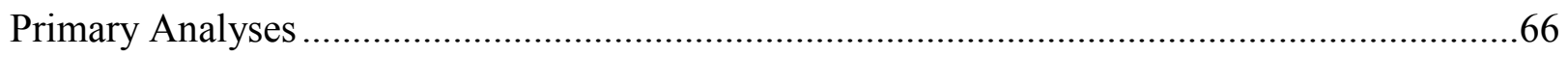

Exploratory Variables: Social Desirability and Depression .............................................68

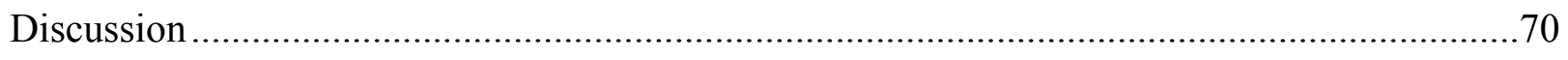

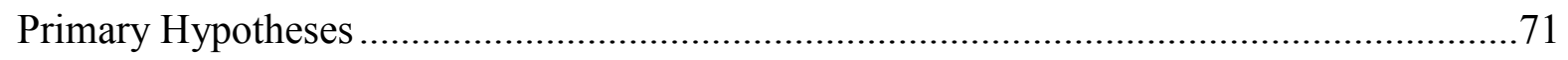

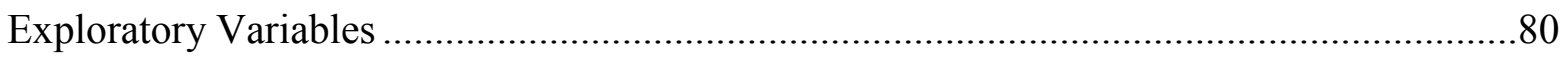

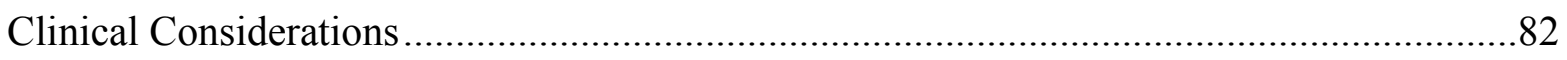

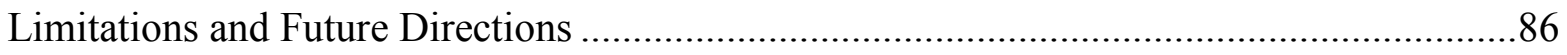


Summary and Conclusion ..................................................................................... 90

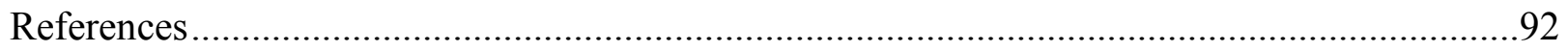

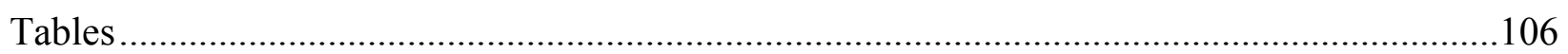

1 - Demographic Information for the Entire Sample ..................................................106

2 - Demographic Variables Broken Down by Faking and Non-faking Groups....................107

3 - BeanFest Game and Test Phase Variables Using the Simplified Version......................109

4 - Correlational Matrix of BeanFest Variables and Participant Demographic Information 110

5 - Comparison of Faking and Non-faking Participants across Main Outcome Variables ....111

6 - Correlational Matrix Including CAPI Lie Scale and Study Variables ...........................113

7 - Stepwise regression predicting CAPI Lie Scale from significant predictors...................114

8 - Stepwise regression predicting CAPI Lie Scale from primary outcomes.......................115

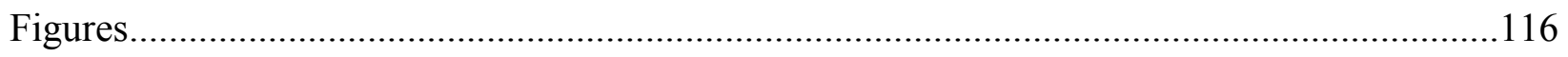

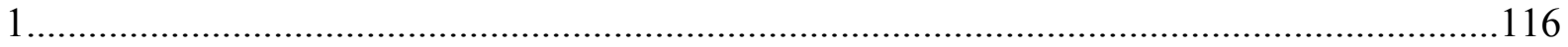

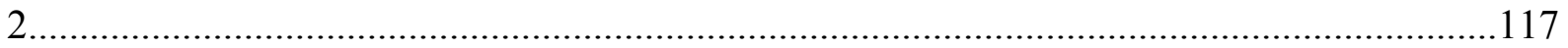

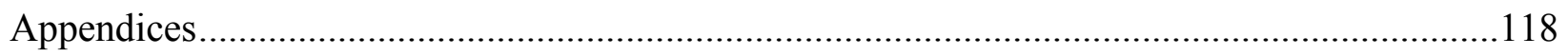

A - Sample Permission to Contact Flyer .............................................................. 118

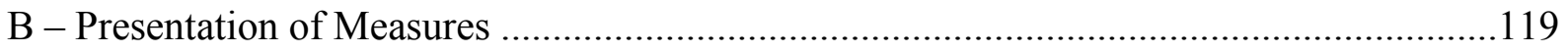

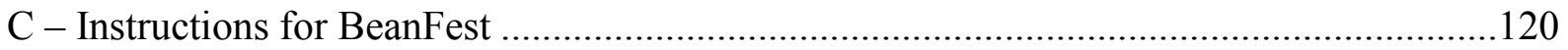

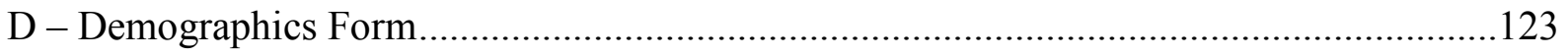




\section{Introduction}

\section{Predicting Child Maltreatment Recidivism}

An integral component of child maltreatment investigations is the parenting capacity assessment (PCA). PCAs are typically ordered by Child Protective Services or the court system to determine whether or not a parent can safely and effectively care for his/her child, using a minimal parenting competency criterion (i.e., whether or not the parent can meet the basic physical and emotional needs of the child; Budd, 2001; Choate, 2009). There is a high likelihood that information derived from PCAs will be used in court proceedings, and recommendations taken from these evaluations are seriously considered by the legal team involved in the case (Budd, 2001; Choate, 2009). A comprehensive and valid PCA is intended to offer multidisciplinary team members information across a variety of domains pertinent to the parentchild relationship, including identifying a parent's current, as well as future functional abilities (e.g., what the parent can do to effectively parent his/her child), and attempting to predict risk for abuse recidivism (Budd, 2001; Kalich, Carmichael, Masson, Blacker, \& Urquiza, 2007). PCAs are often framed as skills-based assessments, and data from these assessments yield specific and clear recommendations addressing what the parent needs to learn in order to safely and appropriately care for his/her child (Azar et al., 1998).

Evaluations carry serious consequences such as referral of families for treatment, settling child custody issues, ensuring the child's protection, and in some cases, termination of parental rights (Azar et al., 1998; Haskett, Scott, \& Fann, 1995). The process of evaluating parents and determining their rights can be lengthy (e.g., some cases have taken an average of 4.4 years to resolve; see Azar et al., 1998; Jellinek, Murphy, Poitrast, Quinn, Bishop, \& Goshko, 1992), and the burden of performing a PCA usually falls on mental health professionals, with many 
professionals reporting that gathering accurate, unbiased information from these evaluations is one of the most difficult aspects of their job (Otto, Edens, \& Barcus, 2000). Therefore, given the time-consuming and often combative nature of PCAs, this evaluation is usually stressful for both the referred parent and mental health professional; however, it is imperative that PCAs gather complete and correct information about the parent and his/her functioning, to make the most accurate and helpful impressions and recommendations possible.

Guidelines have been established by researchers and other organizations to define "best practice" methods for eliciting information during PCAs. Many years of research on PCAs, including ethical guidelines developed by the American Psychological Association (APA, 2013), have identified "best practice" PCAs as comprehensive assessments, using multiple sources and methods, including review of records, clinical interview, psychopathology measures, risk assessment measures, and behavioral observation (Azar et al.,1998; Barone, Weitz, \& Witt, 2005; Budd, 2001; 2005; Budd et al., 2011; Carr et al., 2005; Rohrbagh, 2008; Urquiza \& Blacker, 2005). Mental health professionals who conduct PCAs are also recommended to seriously consider the culture, social class, and environmental factors which may influence the parent's caretaking strategies and beliefs (Azar et al., 1998). Unfortunately, PCAs are often confounded by multiple sources of error and/or bias, which increase the difficulty of conducting a high-quality, comprehensive assessment.

Potential error and/or bias may be derived from the setting of the evaluation, the examiner conducting the evaluation, and/or the referred parent involved in the PCA. In regard to the evaluation setting, parents may be caught in a "coercive context" (Budd, 2001) in which they feel pressure to minimize their report of negative behavior, or respond in a socially desirable manner on self-report measures or during behavioral observation (Budd \& Holdsworth, 1996). 
Indeed, the potential consequences of PCAs, including the possibility for termination or significant modification of parental rights often yields a highly stressful situation. In regard to the examiner who conducts the evaluation, as noted above, these professionals may not consider the culture or context of the parent's behavior and decision-making, and thus may impose their own biases as to what constitutes "good" or "normal" parenting based on their own cultural experience and upbringing. This biased view may potentially lead to impressions and recommendations that do not necessarily reflect the implementation of a "minimal parenting criterion" (Azar et al, 1998; Budd \& Holdsworth, 1996).

To further confound information gathered from PCAs, parents involved in these evaluations may present with low intellectual functioning, and research has demonstrated that individuals with intellectual disabilities may be more likely to engage in socially desirableresponding within psychiatric and forensic contexts, than individuals without a disability (Langdon, Clare, \& Murphey, 2010; Sturmey, 2007). Parents with low intellectual functioning may have key skills deficits, including greater difficulty with perspective taking, working memory, flexibility in thinking, problem-solving skills, and emotion recognition, compared to parents whose intellectual functioning is within normal limits (Azar, Robinson, \& Proctor, 2012). Parents with low intellectual functioning may also present with lower social support and greater interpersonal stress than their peers, as well as a heightened lack of trust in the child welfare system (Azar et al., 2012). These skill deficits, as well as mistrust in the child welfare system may serve to increase the likelihood of engaging in distorted (i.e., invalid) responding during the PCA. Additionally, parents with low intellectual functioning may be at greater risk to experience stigma from mental health professionals regarding their capabilities as a parent (Budd \& Holdsworth, 1996), possibly leading to an increased likelihood of having their parental rights 
terminated (Budd \& Holdsworth, 1996).

\section{Use of the Child Abuse Potential Inventory in PCAs}

As mentioned previously, risk assessment measures have been identified as a key component of a "best practice" PCA. One such measure that is widely used in PCAs is the Child Abuse Potential Inventory (CAPI; Milner, 1986). The CAPI is a 160-item self-report measure that contains six abuse risk domains (distress, unhappiness, rigidity, problems with child/self, and problems from others); data from which are then pooled into a broadband abuse risk score (Milner, 1986). The CAPI was developed primarily to assess future risk (over actual recidivism) of child physical abuse, but has since been used with parents presenting for a range of punitive, coercive, and inappropriate parenting behaviors. It remains one of the few measures used in PCAs that has been normed on the child welfare population, as well as one of the most researched parenting assessment measures (Choate, 2009; Ellis, 2001). The CAPI is written at a third-grade reading level and employs a forced-choice "yes/no" format. Parents may either complete the CAPI themselves or the items can be read out loud by a trained researcher or clinician. Administration instructions require that administrators emphasize the importance of answering questions in an honest manner prior to administering the measure (Milner, 1986).

The CAPI has been found to have sound psychometric properties including strong construct validity (Haskett, et al., 1995). Additionally, it has shown associations with related risk variables including parental history of maltreatment, parent-child conflict, authoritarian parenting style, and parent psychopathology (e.g., distress and low self-esteem; Aragona, 1983; Fulton, Murphy, \& Anderson, 1991; Haskett et al., 1995; Miller, Handal, Gilner, \& Cross, 1991; Milner, Charlesworth, Gold, Gold, \& Friesen, 1988; Stringer \& LaGreca, 1985). The CAPI also has evidence of predictive validity using its broadband abuse risk score (see Chaffin \& Valle, 
2003; Milner, 1994).

Perhaps one of the strongest features of the CAPI is that, in addition to its abuse risk subscales, it also includes validity scales. There are three validity scales (Lie, Random Responding, and Inconsistency), as well as three validity indices (Faking-good, Faking-bad, Random response) developed for the measure, which are utilized to address response distortion (Milner, 1986). The Lie Scale assesses for socially desirable responding, and the Random Responding Scale assesses for confusion or disregard for item content. Furthermore, the Inconsistency Scale specifies random from non-random responding (Milner, 1986). In addition to the Lie Scale, the Faking-good Index of the CAPI was developed to identify parents who deliberately engage in socially desirable responding, outside of chance. The Faking-good Index is considered to be invalid when the Lie Scale is above the clinical cutoff (e.g., a 7 or 8, based on the level of education of the parent) and the Random Responding Scale is 6 or below (i.e., below the clinical cutoff). The authors of the CAPI have provided extensive empirical support for the development of each scale (Milner, 1986), but given its relevancy to the current study, only the Lie Scale and Faking-good index will be emphasized in this literature review.

\section{Development of the Child Abuse Potential Inventory Lie Scale and Faking-good Index}

The Lie Scale on the CAPI was developed from multiple studies using a range of populations. An early study used an undergraduate population and administered 47 items tapping socially desirable responding. Examples of the items were, "I never lose my temper" and "I always tell the truth" (Milner, 1982; 1986). From this study 30 items were chosen. A follow-up study conducted with general population parents from a parent-teacher association in North Carolina further reduced the number of items to 18 ; items chosen were similar in content to the original 47 items (e.g., "I never raise my voice in anger"; see Milner, 1982; 1986). 
Next, the 18 chosen items were integrated into the full measure, adding to a total of 160 items. The CAPI was then administered to a "normal" parent population (e.g., parents without a history of child maltreatment who were also not seeking any treatment services). Parents were required to answer in the forced-choice "yes/no" format used on the current version on the CAPI. During this study Milner et al. (1986) identified a cutoff of 7 to determine socially desirable responding. It was also determined that parents with less than a twelfth grade education would have a cutoff of 8 to be sensitive to potential reading comprehension differences. Overall, higher scores on the Lie Scale were identified as parents who were high on socially desirable responding (Milner, 1986).

Finally, construct validity of the scale was assessed by Robertson and Milner (1983). In this study undergraduate participants were administered the CAPI, a modified version of the Minnesota Multiphasic Personality Inventory (MMPI; Hathaway \& McKinley, 1943) and the Barron ego-strength scale (Barron, 1953). Scores on each measure were compared to determine the utility of the CAPI in identifying socially desirable responding. In this study the CAPI was able to correctly identify $84-95 \%$ of participants with an elevated Lie scale. The authors were able to replicate these results in a similar study using a normal parent (e.g., parents who were not treatment seeking and not involved in the child welfare system) sample (Robertson \& Milner, 1985a).

In addition to these early psychometric studies, Milner and Crouch (1997) examined (a) the impact of response distortion (e.g., faking-good, faking-bad, and inconsistent responding) on scores on the CAPI, Parenting Stress Index (Abidin, 1995) and Adult/Adolescent Parenting Inventory (Bavolek, 1984), and (b) to see if there were differences in the sensitivity of these measures in detecting invalid responding. The authors also compared parents in a "general 
population" group $(n=106)$ and an "at-risk" group $(n=80)$ on how well they could distort their responses on each measure. General population parents were defined as individuals who were not involved in any treatment services, including the child welfare system. At-risk parents were defined as individuals who were enrolled in a parenting group specifically designed to target risky parenting practices. About $80 \%$ of at-risk parents had at least one documented case of physical abuse, and thus the majority of this sample was already offenders. To investigate the ability of the CAPI in being able to detect response distortion, all parents were given four conditions: a "fake good," "fake bad," "respond randomly," or "answer honestly" condition. For all measures (including the CAPI), scores were artificially lower in the "fake good" condition, demonstrating the impact of deception on data gathered from these assessments. The authors also noted that the CAPI Faking-good Index had a much higher rate of correctly identifying fakinggood profiles ( 80 and 88 percent of at-risk and general population parents, respectively) compared to the Parenting Stress Index’s Defensiveness scale (32 and 41.2 percent, respectively) (Milner, 1986). The Adult-Adolescent Parenting Inventory was not assessed in this comparison because it does not contain validity scales.

Although there is a strong empirical base demonstrating the psychometric properties of the CAPI validity scales, including the Lie Scale and Faking-good Index, few studies have examined clinical applications of the validity scales, such as parental characteristics which may differentiate parents with and without an elevated Lie Scale and Faking-good Index (Bradshaw, Donohue, Cross, Urgelles, \& Allen, 2011; Budd et al., 2000; Carr et al., 2005; Costello \& McNeil, 2014). Additionally, although the developers of the CAPI have found that about 7-11\% of general population parents (i.e., parents who are non-treatment seeking without a report of maltreatment) "fake good" on the CAPI (Milner, 1986), studies using other populations (e.g., 
parents involved in the child welfare system) have not been able to clearly identify a concrete percentage of individuals who engage in socially desirable responding. In fact, across studies, the range of invalid profiles has varied from 19\%-58\% (see Bradshaw et al., 2011; Budd et al., 2000; Carr et al., 2005; Costello \& McNeil, 2014).

One potential reason for this range in responding may be due to the "coercive context" (Budd, 2001) of the assessment or research study. Specifically, parents without a treatment history may feel less inclined to present themselves in a socially desirable manner compared to parents who are involved in the child welfare system, due to the lack of risk regarding their parental rights. Or, parents who are involved in the child welfare system may simply have different characteristics compared to parents with no systems involvement (e.g., lower intellectual functioning, higher stress) that may impact scores on the Lie Scale and Faking-good Index.

There have been no published studies utilizing the CAPI which have focused solely on treatment-seeking, non-maltreating parents. Participants have either been undergraduates or “general population" parents (Milner \& Crouch, 1997; Robertson \& Milner; 1983; 1985b) or parents who already involved in the child welfare system (Bradshaw et al., 2011; Budd et al., 2000; Carr et al., 2005; Costello \& McNeil, 2014). Because there is a gap in this literature, it has only been possible to report on parents who are on opposite ends of a continuum. To better understand subtle characteristics of parents involved in the child welfare system, it appears important to understand how these parents compare to parents who may demonstrate less serious risk behaviors but who may still feel pressure to present themselves in a socially desirable manner.

Finally, and possibly most importantly, as stated previously, few studies have sought to 
investigate which specific parenting characteristics are associated with an elevated Lie Scale and Faking-good Index on the CAPI, despite recognition of the need for this information from experts in the field (Budd \& Holdsworth, 1996). Better understanding of why parents engage in socially desirable responding on the CAPI may serve to decrease examiner bias, and inform "best practice" assessment parameters, meant to engage parents and reduce the tendency for parents to feel pressure to present themselves as "superparents" (Gardner, 1982). It is argued that without knowledge of why parents engage in socially desirable responding on the CAPI, professionals may be more prone to identify these individuals as "manipulative" or "liars," which may color how data and treatment recommendations are presented in their report, therefore increasing the risk for stigma and bias. Indeed, parents who engage in socially desirable responding may have skills deficits in other, key areas of intervention, such as intellectual functioning, lack of social support, or an unrealistically positive perception of the world, which may affect how they react and respond during PCAs.

For example, a parent with low intellectual functioning coupled with a highly positive view on the world may be very different, and need different services than a parent who is truly attempting to manipulate and lie during evaluations. However, without knowledge as to what constitutes invalid responding during PCAs, professionals are left to tease apart these behaviors in an already highly stressful and complex situation. Currently, there have only been four published studies directly examining factors associated with socially desirable responding using the CAPI Lie Scale and/or Faking-good Index (Bradshaw et al., 2011; Budd et al., 2000; Carr et al., 2005; Costello \& McNeil, 2014), and these studies are described below. Although data from each investigation have provided a solid foundation on which to build further research, the number of studies is low, and there are limitations present in each, proving the need for more 
research in this area.

\section{Review of the Current State of the Literature}

Outcomes of parental faking-good responding. Bradshaw et al. (2011) investigated the influence of faking-good responding on parent report of satisfaction and child maltreatment potential using data collected from 82 mothers who were referred for treatment to the local Department of Family Services agency due to neglect and substance use disorders. In their sample 58 percent of the participants had an invalid CAPI profile due to an elevated Faking-good Index. Respondents in the faking good group reported both lower risk for abuse and higher parental satisfaction than parents with valid profiles. Additionally, when the authors only analyzed data from respondents with valid profiles on the CAPI, they found parents indicated higher levels of parenting distress and unhappiness; however these results were diminished when scores from socially desirable parents were added to the analyses. The authors concluded that the addition of invalid profiles on the CAPI may yield "guarded information" (Bradshaw et al., 2011; pp. 548) when interpreting results from assessments, and these artificially inflated scores may result in missed opportunities to ask pertinent follow-up questions or recommend helpful interventions for the parent and child. Therefore, although these authors did not examine which specific parental characteristics yielded socially desirable responding on the CAPI, they demonstrated the significant impact faking good responding can have on the measure, thus providing good evidence as to why further investigation of faking-good responding is warranted.

Parental cognitive functioning. Two published studies have addressed the impact of parents' cognitive functioning (i.e., intelligence and reading comprehension) on responding during parenting-related evaluations (Budd et al., 2000; Carr et al., 2005). As stated previously, individuals with lower intellectual functioning, lower educational level, and lower 
socioeconomic status present to the child welfare system at greater rates than their counterparts and have historically experienced stigma from mental health professionals (Azar et al., 2012; Budd \& Holdsworth, 1996; Carr et al., 2005). These parents are typically asked to complete selfreport measures during PCAs, which require them to reflect on their own attitudes and behavior in a critical manner, as opposed to judgment-based or behavioral tasks (Norris, Larsen, Crawford, \& Cacioppo, 2011). Parents who may have deficits in executive functioning (e.g., problem-solving, critical thinking skills, introspection) may have difficulty in successfully completing these measures, thus leading to a potential of distorted responding and invalid profiles.

Carr et al. (2005) examined socially desirable responding in parents undergoing a PCA at the Family Court Centre, an agency which primarily administered court-ordered parenting evaluations. Participants in this sample were given the MMPI-2 (Butcher, Graham, Ben-Porath, Tellegen, Dahlstrom, \& Kaemmer, 2001), the Personality Assessment Inventory (Morey, 1996), and the CAPI (Milner, 1986) to assess for faking-good responding. They also received the Child Behavior Checklist (Achenbach, 1991) to rate their child's behavior. Parent intelligence was assessed by using the Wechsler scales (Psychological Corporation, 1997; 1999). Approximately sixty percent of parents in the sample had either an elevated L or F scale on the MMPI-2, which yielded lower scores on the clinical scales (e.g., indicating better psychological and behavioral functioning). Approximately twenty percent of parents in the sample had a PAI with an elevated positive impression management scale. Additionally, forty-nine percent of parents had an invalid profile on the CAPI due to faking-good responding (as measured by the Faking-good Index). Consistent with the Bradshaw et al. (2011) study, parents with a faking-good profile on the CAPI yielded an overall lower abuse risk score, as well as lower scores on the Distress, Unhappiness, 
Ego Strength, and Loneliness subscales than parents without a faking-good profile.

Interestingly, few differences were found in faking-good or socially desirable responding when comparing parents with an IQ of 80 and above to parents with an IQ below 80 on the Wechsler scales. No evidence supported that parents with a lower IQ had significantly different scores on the $\mathrm{L}$ and $\mathrm{K}$ (socially desirable responding and defensiveness, respectively) scales on the MMPI-2, the positive impression management scale on the PAI and the Faking-good Index on the CAPI. Parents only differed by intelligence on the F (inconsistency) scale of the MMPI-2; parents with a lower IQ were found to be more likely to have an elevated F scale on this measure. Therefore, parents with an IQ in the below average range were still able to complete these self-report inventories without a significantly different response bias from their counterparts with a higher IQ. Although this study is one of the few to investigate the role of parent intellectual ability during PCAs, there are some limitations which should be noted. Parents were tested on reading comprehension ability but this information was not reported. Therefore, parents may have been able to cognitively understand the measures administered, but may not have been able to fully comprehend each item. Additionally, the authors did not report how the measures were administered (e.g., either orally or via paper-pencil format) which may have affected ability to respond.

Reading comprehension, but not intelligence was examined in a study completed by Budd et al. (2000). The authors originally assessed for specific risk factors related to child abuse, but expanded the scope of their study after identifying that $19 \%$ of mothers $(n=14)$ had an invalid faking-good profile on the CAPI (Budd et al., 2000). Participants in the study were 75 adolescent mothers who were wards held by the Illinois Department of Children and Family Services (Budd et al., 2000). Mothers were recruited by caseworkers or therapists in private 
practice and had retained their parental rights during the time of the study. Therefore, they did not experience any direct threats to their parenting rights outside of necessary mandated reporting required of researchers entering their homes.

At pretreatment the adolescent mothers were administered measures assessing range of individual characteristics associated with child maltreatment risk. These measures included the Wide Range Achievement Test-Revised (Jastak \& Wilkinson, 1984) to test for reading comprehension, the Parent Opinion Questionnaire (Azar, Robinson, Hekimian, \& Twentyman, 1984; Azar \& Rohrbeck, 1986) to examine unrealistic parenting expectancies, the Arizona Social Support Interview Schedule (Barrera, 1981) to assess for an individual's exposure to social contacts across a range of settings, and the Symptom Checklist 90-Revised (Derogatis, 1983) to identify parent psychopathology. Although all measures were administered orally to control for reading proficiency, faking-good mothers scored lower on reading comprehension than honest mothers. This was the only difference between the two groups. This study is strong in that it is assessed a wide range of factors potentially associated with socially desirable responding on the CAPI. However, like the Carr et al. (2005) study, limitations remain. The authors did not include a measure of IQ in their data collection, and thus parent IQ, as well as reading comprehension, may have impacted results.

A recent article (Costello \& McNeil, in 2014) attempted to also examine differences in parental characteristics between parents with a faking-good profile versus parents with an "honest" profile on the CAPI. One of the limitations of the investigation was that the authors used secondary analyses, thereby making the investigation a retrospective study. The original data were obtained by the National Data Archive on Child Abuse and Neglect through Cornell University. Data had been collected by a study conducted at the University of Oklahoma Health 
Sciences Center (see Chaffin, Silovsky, Funderburk, Valle, Brestan, Balachova, Jackson...et al., 2004) funded by the United States Department of Health and Human Services, Administration on Children, Youth and Families, Children's Bureau, Office on Child Abuse and Neglect. The investigation was a treatment outcome study examining abuse recidivism and Parent-Child Interaction Therapy (McNeil \& Hembree-Kigin, 2010). Parents who had a report of physical abuse or neglect with an IQ of 70 or above who were able to provide consent for participation were included in the study (Chaffin, 2004).

After recruitment and screening 110 parent participants ( 71 mothers and 37 fathers) were included in the original Chaffin et al. (2004) study. In this sample $23.6 \%$ of parents had an invalid profile due to faking-good responding (Costello \& McNeil, in 2014). Contrary to the Carr et al. (2005) study, parents with an invalid profile due to faking-good responding on the CAPI did differ by IQ (measured by the Kaufman scales; Kaufman \& Kaufman, 1990). Specifically, parents who had a lower IQ than their counterparts were more likely to have an invalid profile due to faking-good responding. But, similar to the Carr et al. (2005) study, Chaffin et al. (2004) did not describe the reading ability of parents and did not include information about how the measures were administered. Thus, these variables could not be assessed in the Costello and McNeil (2014) paper.

In sum, across the three published studies investigating the potential impact of parental intelligence and/or reading comprehension on responding on the CAPI (Budd et al., 2001; Carr et al., 2005; Costello \& McNeil, 2014) little can be concluded concerning their effect on the Faking-good Index. Carr et al. (2005) found that parents did not necessarily need to demonstrate lower intelligence to fake good on the CAPI, but Costello and McNeil (2014) contradicted these results. Neither study tested parents on reading comprehension. When tested for reading 
comprehension, Budd et al. (2000) found that mothers with an invalid profile due to faking-good responding had lower scores than mothers with valid profiles. But, the authors did not test for intellectual functioning in their study. Replication via additional research studies, then, is needed to test the role of intellectual ability and reading comprehension on responding on the CAPI.

Psychopathy. A notable limitation in the CAPI literature is that no published studies have directly examined parental characteristics associated with manipulation and lying. However, by definition, the Faking-good index on the CAPI attempts to measure parents who are purposefully (rather than accidentally) distorting their responses to appear socially desirable (Milner, 1986). Anecdotally, parents with faking-good profiles on the CAPI are typically viewed as individuals who possess the intellectual ability to understand how to purposefully lie and manipulate on the measure, but these parental characteristics have not directly been examined by researchers conducting investigations in this area. It should be noted that researchers have identified that higher levels of psychopathic traits, as measured by the Levenson Self-Report Psychopathy Scale (Levenson, Kiehl, \& Fitzpatrick, 1995) and Psychopathic Personality Inventory (Lilienfeld \& Andrews, 1996) have been associated with a greater tendency to fake good on self-report personality measures (e.g., the Holden Psychological Screening Inventory; Holden, 1996), but, these studies were conducted with undergraduate students, and were not related to parenting. It appears that no studies have been conducted assessing psychopathy in the context of faking-good responding on parenting measures. Indeed, a parent who purposefully "fakes good" on the CAPI may be quite different from a parent with low intellectual functioning, who may be unable to detect the nuances of the items on the CAPI, and treatment recommendations may shift based on these parental differences.

Therefore, it is important to assess for parental characteristics of psychopathy, when 
attempting to understand why parents engage in faking-good responding on the CAPI. Although there is a debate around the exact definition and behaviors associated with psychopathy (Skeem, Polaschek, Patrick, \& Lilienfeld, 2011), years of research have identified operationalized traits associated with this profile. The foundation for what defines psychopathy has been attributed to Cleckley's (1976) diagnostic criteria for psychopathy, including three major domains: a) positive adjustment (e.g., superficial charm, absence of delusions and neurosis), b) behavioral deviance (e.g., impersonal relationships with others, poor judgment, lack of motivation to follow-through with goals and/or learn from mistakes), and c) emotional-interpersonal deficits (e.g., lack of remorse, insincerity, failure to truly love others, poor insight) (Skeem et al., 2011), which have since been adapted through years of assessment and research.

Modern definitions of psychopathy have largely been driven by data gathered from assessment measures targeting this construct. The most widely used and validated measure of psychopathy is the Psychopathy Checklist - Revised (Hare, 1991), a 20-item clinician rating scale, which was developed to study personality characteristics (as opposed to behavioral patterns) associated with psychopathy (Skeem et al., 2011). "Best practice" data collection using the PCL-R includes interviewing the individual, as well as collecting data from collateral sources, including record review; however, data can be exclusively collected by review of records (Skeem et al., 2011). Items associated with the concept of psychopathy were derived both from the foundational Cleckley (1976) criteria, as well as Hare's own clinical experience (Hare \& Neumann, 2008).

From this information, a two-factor model, identifying primary and secondary psychopathy (which includes four facets) evolved. The PCL-R defines psychopathic personality characteristics as falling into either Factor 1 (primary psychopathy): interpersonal-affective 
scale, or Factor 2 (secondary psychopathy): antisocial scale. The two-factor model of the PCL-R was designed in such to parse out individuals with true psychopathy from individuals with a diagnosis of Antisocial Personality Disorder (Levenson et al., 1995). The interpersonal-affective scale is further divided into two facets: interpersonal (e.g., pathological lying, superficial charm, manipulative) and affective (e.g., lack of remorse, callousness, failure to accept responsibility for actions). Additionally, the antisocial scale is also divided into two facets: lifestyle (e.g., need for stimulation, no long-term goals, impulsivity) and antisocial (e.g., early behavior problems, including juvenile delinquency) (Hare, 2003; Skeem et al., 2011). As stated above, parents who present with faking-good profiles on the CAPI are often compared to individuals who meet criteria for Factor 1 (interpersonal-affective) on the PCL-R. Specifically, individuals with these CAPI profiles are anecdotally described as manipulators and liars, and who fail to take responsibility for their parenting. However, this classification comes with serious consequences, as labeling these parents as individuals as having a set of ingrained, pathological personality characteristics may lead to bias and misinterpretation of information during a PCA.

Although the PCL-R is considered the most widely used and validated measure of psychopathy, the actual completion of the measure can be a lengthy process, with the necessary data collection to complete the measure as long as three hours to complete (Skeem et al., 2011). Therefore, self-report measures of psychopathy were developed to streamline the process, while still screening for psychopathic personality characteristics. Two notable self-report measures are the Psychopathic Personality Inventory - Revised (PPI-R; Lilienfeld \& Widows, 2005) and the Levenson Self-Report Psychopathy Scale (LSRP; Levenson et al., 1995). Typically, these selfreport measures have been used with non-criminal and/or non-institutionalized samples. The PPI-R has been noted to be the most widely used self-report measure, although the LSRP has 
also been used in research and clinical work. The PPI-R is a 154-item measure which is based largely off of the Cleckley (1976) criteria for psychopathy, and consists of three factors: fearless dominance (e.g., narcissism, reduced empathy), impulsive antisociality (e.g., impulsivity and aggressiveness), and coldheartedness (Skeem et al., 2011).

By contrast, the LSRP was based off of the two-factor model of the PCL-R (Hare, 1991; 2003), to identify characteristics of primary and secondary psychopathy (i.e., Factors 1 and 2 on the PCL-R, respectively), in a non-institutionalized sample. Consistent with the design of the PCL-R (Hare, 1991), the LSRP was developed to be able to identify characteristics of psychopathy (e.g., lying, manipulation, lack of remorse) in individuals over and above antisocial behaviors and personality traits (e.g., impulsivity, poor frustration tolerance, lack of future planning; Levenson et al., 1995). The LSRP was originally normed on an undergraduate student population, and factor analysis on the original set of items yielded a two-factor model, which is organized similarly to the PCL-R, containing a primary and secondary psychopathy scale. Compared to the PPI-R (Lilienfeld \& Widows, 2005), it is a briefer self-report measure, with 26 items rated on a 1 (strongly disagree) to 4 (strongly agree) Likert scale. Since its initial development, the LSRP was been widely used with both non-institutionalized and offending populations, and has demonstrated sound psychometrics across research studies (Lynam, Whiteside, \& Jones, 1999; Reidy, Zeichner, \& Martinez, 2008). Given its relatively short time to complete, and its history of use with a non-criminal, noninstitutionalized population, it was chosen to assess for characteristics of psychopathy in parents in this current study.

As mentioned previously, researchers have established that, although characteristics of psychopathy and Antisocial Personality Disorder (ASPD) are associated with each other, psychopathy has been argued to be a distinct set of personality characteristics, while ASPD is 
thought to be comprised of maladaptive behaviors (Skeem et al., 2011). Furthermore, individuals with psychopathic characteristics may not necessarily engage in antisocial or criminal behaviors. Therefore, it is important to tease apart these differences in definition (as well as the controversy surrounding the distinct differences between psychopathy and ASPD) when applying these concepts to parents in the child welfare system.

With this concept in mind, the only published study to attempt to assess the concept of maladaptive behaviors in relation to faking-good responding is the paper by Costello and McNeil (2014). It is important to note that these authors examined differences in parents with and without an elevated Faking-good Index on the CAPI by antisocial behaviors (not psychopathy) using items on the Diagnostic Interview Schedule Antisocial Personality Disorder module (Robbins, Helzer, Croughan, \& Ratcliff, 1981). Items on this module include past behavioral problems with lying, stealing, vagrancy, arrests, and starting fights (Chaffin, 2004). No differences emerged on any item between parents with invalid profiles due to faking good responding and parents with valid profiles on the CAPI, and parents who had distorted their responding in this study were no more likely to engage in self-reported maladaptive and/or criminal behaviors than parents with valid profiles. However, given that this study did not include a measure of psychopathy, differences in specific personality traits (e.g., lying, manipulation, lack of remorse), which are typically thought to characterize individuals with faking-good profiles on the CAPI, were not assessed. Therefore, further study of the influence of psychopathy on faking-good responding on the CAPI is warranted.

Positivity bias. Costello and McNeil (2014) identified an additional area of consideration when investigating parental characteristics associated with faking-good responding on the CAPI. The authors proposed that examining a potential attitudinal bias (e.g., a tendency to interpret 
information either predominantly positively or negatively) may be warranted, given many of the abuse risk and validity subscales on the CAPI contain items related to parental attitudes, including distress, happiness, and opinions of children and others. Specifically, the authors proposed that parent faking-good responding on the CAPI may be impacted by a "positivity bias," which can be defined when “...people seek a positive image of themselves and their environment with such vigor that reality at times is selectively interpreted and at other times patently ignored" (Mezulis, Abramson, Hyde, \& Hankin, 2004, pp. 711). This selective interpretation of information may serve a protective or adaptive function, in which selectively attending only to positive or self-serving information in the environment may act as a buffer from reality (Mezulis et al., 2004).

Indeed, early research has suggested that maintaining positive illusions about oneself may not only protect against depressive symptomatology but may lead to a greater sense of control and psychological well-being (Abramson \& Alloy, 1981; Taylor \& Brown, 1988). Perhaps one of the most-researched positivity biases is the self-serving attributional bias, in which individuals attribute positive life events to themselves, and negative events to outside causes (Mezulis et al., 2004). There have been decades of research studying the effects of this attitudinal style and multiple reviews have demonstrated that the self-serving attributional bias is robust and stable (Mezulis et al., 2004 for a complete listing of reviews). However, the stability and universality of the self-serving attributional bias has not been without controversy, and many researchers have called into question its generalizability across populations differing by a multitude of variables, including country of origin, race, ethnicity, age, gender and psychopathology.

A recent review (Mezulis et al., 2004) addressed these concerns by conducting a metaanalysis of 266 published articles in this area. The authors found a very large effect (Cohen's $d=$ 
0.96) using a random-effects analysis of the data, indicating that the self-serving attributional bias was indeed stable across these studies. Furthermore, the authors examined potential moderators (e.g., age, gender, cultural differences, and psychopathology) and found that, typically the self-serving attributional bias is present in both males and females, is most pronounced in childhood, but in males, stays relatively the same over the lifespan (as compared to a marked decrease in this bias for females beginning in adolescence). Additionally, the authors found that this biased cognitive style was largest in the United States and other Western countries and cultures, although all countries showed positivity, rather than, a negativity bias. There were no differences found between ethnic groups within the United States. Finally, individuals without a history of psychopathology had a larger self-serving attributional bias than individuals with such a history. To note, groups with a history of a depressive disorder had the smallest self-serving attributional bias, as compared to groups with other psychological disorders and groups with no psychopathology.

What makes the self-serving attributional bias important to study in relation to fakinggood responding on the CAPI? To begin, as Mezulis et al. (2004) demonstrated, the self-serving attributional bias has been found to be most pronounced in individuals from the United States and other Western countries, and is relatively stable over time (although generally declines over time for women). Given the CAPI has been validated in the United States (Walker \& Davies, 2010), individuals who are given this measure during PCAs may present to the evaluation with an attitudinal bias that could affect how they respond on the measure. Furthermore, many of the items on the CAPI pull for information related to a wide range of personal and world views, which may be affected by an overly optimistic (and potentially unrealistic) view of oneself. Examples of such items from the Lie Scale include, "I am always a good person," and "I always 
do what is right" (Milner, 1986). Additionally, items from other subscales, including "I love all children," "My life is happy," and "I am often depressed" (Milner, 1986) may be artificially endorsed or not endorsed, based on a respondent's attitudinal style.

Although parents who are ordered to a PCA are typically experiencing stress related to the possible removal of their children or even termination of parental rights, the presence of a self-serving attributional bias may buffer them from these stressors, and thus distort how they respond during a PCA. This proposal could be argued as conjecture, given that there are no published studies examining the impact of the valence of an individual's attitudes on responding on the CAPI, so further investigation is warranted. Specifically, parents with an invalid profile on the CAPI due to faking-good responding may have different characteristics than parents who are attempting to manipulate and lie to mental health professionals, which, consistent with the other proposed areas of exploration could in turn, lead to different diagnostic impressions and treatment recommendations.

Only one published study (Costello \& McNeil, in 2014) considered the possible effect of a positivity bias in responding on the CAPI. This phenomenon was only identified as an area of interest after it was found that parents with an elevated Faking-good Index on the CAPI in the Chaffin and colleagues (2004) study had lower depressive symptoms measured by the Beck Depression Inventory (BDI; Beck, Ward, Mendelson, Mock, \& Erbaugh, 1961) than parents without this elevation. In the context of the self-serving attributional bias discussed above (especially noting that individuals with depression were found to have the lowest incidence of a self-serving attributional bias), the authors of this study proposed that parents in the Chaffin et al. (2004) study could have presented with a tendency to view themselves and their world in a more positive manner, as compared to their peers, which may have elevated the Lie Scale, and 
subsequently the Faking-good index on the CAPI. However, this study derived these observations from the use of a self-report measure of depression, which did not measure the full range of attitudes (e.g., both positive and negative) parents had of themselves and the world.

It could also be argued that parents simply "faked" on the BDI-II in this study, attempting to present themselves as appearing less depressed than parents who answered honestly on the CAPI. Indeed, Field (1995) found that mothers who faked good on the MMPI-2 were more likely to underreport depressive symptoms on the BDI, despite being less responsive to their children during parent-child interactions. As reported previously, parents in this study did not differ on ASPD behaviors, but given that psychopathy was not directly measured, it cannot be determined that these parents potentially differed in characteristics like manipulation and lying. However, it was proposed that, despite the limitations in assessment, one possible conclusion may have been that faking good parents in the Chaffin et al. (2004) study were experiencing a positivity bias that "honest" parents did not have. To best investigate this phenomenon, more research should be conducted using a non-face valid measure of attitude formation and generalization.

\section{Considerations When Addressing Socially Desirable Responding}

Examination of the current state of the literature has identified that faking-good responding affects the quality and validity of information derived from PCAs, and researchers in this field have acknowledged that, although specific parental characteristics may be associated with invalid profiles on the CAPI, these profiles have not yet been identified (Budd \& Holdsworth, 1996; Carr et al., 2005). However, despite this acknowledgement, only a handful of studies have been published in the last thirty years (since the CAPI manual was first published; Milner, 1986) which have attempted to understand which parental characteristics may serve to 
affect responding on risk assessment measures like the CAPI. From these studies, several "areas of exploration" have emerged, which are described below. Additional research is essential in understanding these areas, so that the structure, content, and focus of PCAs can be adapted in a way to obtain the most valid information possible from parents. Given the CAPI is typically used in PCAs, understanding which parental characteristics may affect faking good responding on this measure may help to understand how to best conduct PCAs, as well as to provide treatment recommendations that are most appropriate for the referred parent.

The first area of exploration is type of parent, or context of assessment. Milner and Crouch (1997) demonstrated that both "general population" and "at-risk" parents could effectively distort their responding on the CAPI. But, because no other studies have compared the type of parent participant, and published studies on the CAPI have only included parents on opposite ends of a risk continuum, replication and expansion is needed. Given the wide percentage (19\%-58\%) of parents who have produced invalid profiles in past studies, differences may arise given the context or what is "at stake" for parents in a particular study. Additional research is needed, then, to move past simply comparing parents with no services involvement to parents with a high level of involvement, to assess for more subtle differences between parents who are involved in parenting programs, but who do not necessarily have an open child protective services case.

The second consideration is parental cognitive functioning, as defined by intelligence and reading comprehension. Parents who have low intellectual functioning may not be able to fully understand the items on measures like the CAPI, especially items that tap high-frequency behaviors over a long period of time (e.g., whether or not spanking is always permitted when disciplining a child, whether or not it is acceptable to pick up a crying child). As a result, these 
increases in the difficulty or the burden of the task may result in less accurate responding (Morsbach \& Prinz, 2006). Parents may also not possess the skills necessary to engage in selfreflection, critical thinking, or introspection, often needed to complete self-report or inventorybased measures (Norris et al., 2011). Thus, parents who appear to be manipulative or who are trying to "cheat" the system, in actuality, may not have the intellectual ability to answer appropriately, but nonetheless, may be labeled as such. It is important to note that high-quality, evidence-based services, targeting behavioral and social cognitive capabilities have been developed for parents with low intellectual functioning and social information processing deficits (e.g., unrealistic expectations, poor problem-solving skills, attributional biases), and parents have responded well to the use of these services (Azar, Stevenson, \& Johnson, 2012 for a review). However, if parents are simply viewed as being "manipulative" or "lying" during PCAs, recommendations for these services may not be made, and parents could potentially miss out on valuable experiences to build their parenting skills repertoire.

In a related area, parents may not possess adequate reading comprehension skills needed to appropriately complete measures. Mothers with a faking-good profile on the CAPI in the Budd et al. (2000) study were found to have lower reading comprehension than mothers with valid profiles. In the Costello and McNeil (2014) study, visual inspection indicated a higher percentage of faking-good parents answered "all or none" questions (e.g., "I love all children") than non-faking parents. Parents in this study may not have been able to understand the nuances of the items on the CAPI and thus may have appeared to harbor attitudes different from their actual perspectives. One major limitation of the existing literature is that in most studies the authors did not specify whether measures were presented in a paper-pencil format or were administered orally. Parents who cannot comprehend items or who read items as literal, "black 
and white" concepts may have a harder time understanding items on risk assessment measures.

The third area of exploration that remains important is whether or not parents demonstrate psychopathy (e.g., manipulation, lying, superficial charm) during evaluations. Given the high-risk nature of evaluations it appears intuitive that parents would attempt to make themselves look better, or "minimize their personal and family problems" (Milner \& Crouch, 1997, pp. 633) while completing measures like the CAPI. Inherent in these behaviors is the fact that parents need to be savvy enough to know how to respond and behave in front of mental health professionals. This runs counter to parents who have low intellectual functioning, and who may not know how to make themselves look socially desirable, and therefore it is important to investigate this possible distinction in the context of a PCA.

Finally, results from the Costello and McNeil (2014) study raised a fourth area of exploration. In their investigation, parents with invalid profiles on the CAPI were found to have lower depression scores on the Beck Depression Inventory (Beck et al., 1961) than parents with valid profiles. Although it could be argued that parents were simply "faking" when completing the BDI, another interpretation is that parents with a faking-good profile may have demonstrated a greater tendency to maintain unrealistically high perceptions of themselves, their children, and their parenting. Presently there is a large literature base examining the role of positive illusions on depressive cognitions and decision making. Although there have been some discrepancies in the literature, authors have generally agreed that individuals who harbor positive illusions may experience a temporary or even a prolonged or pervasive relief from experiencing negativity in their environment (Colvin \& Block, 1994; Taylor \& Brown, 1988). Additionally, individuals who are non-depressed have been shown to rate themselves more positively than independent observers (Lewinsohn, Mischel, Chaplin, \& Barton, 1980). Therefore it is important to 
investigate if parents who present themselves in a socially desirable manner on the CAPI simply experience less depressive symptoms and more positive illusions than parents who present themselves honestly. Perhaps positive illusions are adaptive coping skills for parents who are often in environments that are highly chaotic and negative.

One unique way of assessing parent attitude formation is the use of the BeanFest paradigm. BeanFest is a performance-based measure used to assess learning, attitude generalization, and approach/avoid behavior in novel situations by exposing participants to stimuli (i.e., positive and negative "beans") to which they have not previously encountered (Fazio et al., 2004). BeanFest is unique because participants do not have any prior emotional association or knowledge of the stimuli before they begin the task. Therefore, it can be considered a non-face valid measure of learning, attitude generalization, and approach/avoid behavior.

Through replication, three patterns of behavior have been identified. These patterns have been classified as the sampling bias, the weighting bias, and the learning bias (Shook, Fazio, \& Vasey, 2007). The sampling bias has been defined as the extent to which individuals approach or avoid perceived negative or positive beans. Specifically, studies utilizing the BeanFest have found that participants were more likely to avoid beans that were either learned or perceived to be negative, following a "risk aversion" phenomenon (Kahneman \& Tversky, 1988). Individuals demonstrating a sampling bias typically tend to learn negative beans better than positive beans during a contingent feedback (e.g., feedback is only given to the participant if he/she approaches the bean) task (Shook et al., 2007). The weighting bias, most consistent with attitude generalization to new stimuli, measures the extent to which individuals classify (or weight) novel or unfamiliar stimuli as either positive or negative. Studies using the BeanFest have typically 
found that participants tended to rate novel beans as negative, rather than positive (i.e., essentially weighting beans with a negative valence more than beans with a positive valence). Lastly the learning bias has been defined as the tendency for individuals to learn beans of a particular valence (e.g., negative beans) more strongly than other beans (e.g., positive beans). It is important to note, that although this study proposes to examine a positivity bias in learning beans during BeanFest, past research has found that participants generally have demonstrated a negativity bias (i.e., the tendency to learn and remember negative beans better than positive beans). This phenomenon was first identified by Fazio et al. (2004). In the current study, the learning bias and weighting bias variables were assessed in relation to faking-good responding. Therefore, they were emphasized (over the sampling bias) in the review below.

Shook et al. (2007) and Conklin, Strunk, and Fazio (2009) both investigated learning on the BeanFest, in the context of emotional disorders. Shook et al. (2007) recruited a non-clinical sample of 31 female and 22 male college students, who completed the "full-feedback" version of BeanFest (i.e., they received information on each bean, regardless of whether or not they approached or avoided the bean) and three questionnaires assessing for emotional disorders: the Beck Depression Inventory-II (Beck, Steer, \& Brown, 1996), the Beck Anxiety Inventory (Beck, Epstein, Brown, \& Steer, 1988), and the Cognitive Style Questionnaire (Abramson, Alloy, Hogan, Whitehouse, Cornette, Akhavan, et al., 1998). In this study the authors specifically investigated the presence of a learning bias for negative beans. Overall learning for the sample was high, and participants learned the beans above chance. However, although both the positive and negative beans were learned above chance, participants demonstrated better learning of the negative beans, thus indicating the presence of a negativity bias. Additionally, participants with reported more depressive symptoms, a more negative thinking pattern, and heightened anxiety 
symptoms demonstrated a deficit in learning positive beans. By contrast, the presence of emotional distress did not yield a deficit in learning negative beans.

Conklin et al. (2009) sampled 34 college students (17 individuals who met criteria for a depressive disorder on the Structured Clinical Interview for DSM-IV; First, Spitzer, Miriam, \& Williams, 2007, and 17 individuals who fell into the lowest $30 \%$ of scores on the BDI-II (Beck et al., 1996) and Hamilton Rating Scale for Depression (Hamilton, 1960; Williams, 1980), and also administered the full-feedback version of BeanFest. The major goal of this study was to assess how participants learned positive and negative beans based on whether or not they presented with clinically elevated symptoms of depression. Consistent with Shook et al. (2007), participants who had clinically-elevated levels of depression at the start of the study were unable to learn positive beans as well as participants with low levels of depression. A learning bias, favoring better learning of negative beans, was present for the participants who met criteria for a depressive disorder. Within the depressed group, participants with a greater severity of depressive episodes were less able to learn the positive stimuli than participants with less severe depressive episodes (Conklin et al., 2009). Thus, across both studies, the two groups of authors demonstrated a clear learning bias (in favor of learning negative stimuli) and a pronounced deficit in learning positive information for individuals with emotional distress.

Recent studies utilizing BeanFest have also investigated the weighting bias (measuring how attitudes are generalized to new stimuli), rather than the presence or absence of a learning bias (Pietri, Fazio, \& Shook; 2012, 2013). Pietri et al., (2012) conducted a series of experiments using a sample of college students, and had participants complete a short mood questionnaire and a series of anagrams, ranging in difficulty. Participants were also administered the standard, fullfeedback version of BeanFest (i.e., the BeanFest program administered in the original Fazio et 
al., 2004, and subsequent studies) or a simplified, full-feedback (designed to increase learning of both the positive and negative beans, such that the weighting bias could be measured without poor learning as a potential confounding variable) version of the BeanFest. Overall, the authors found that across the standard and simplified versions of the BeanFest, participants who demonstrated a negative weighting bias typically presented with a poorer mood at the end of the study. However, this phenomenon was only present when participants entered the study in a positive mood (thus, demonstrating a potential floor effect for participants entering the study already in a negative mood). Pietri et al. (2013) utilized the standard, full-feedback version of BeanFest (Fazio et al., 2004) with a college-student population, and found that individuals demonstrating a greater negative weighting bias were more likely to be concerned about rejection from others, perceive threat in ambiguous situations, and less likely to take risks. In addition, participants were less likely to feel comfortable encountering new situations (Pietri et al., 2013).

Although there has been a strong literature base clearly demonstrating the presence of a learning and weighting bias in BeanFest, to date, no published studies using the BeanFest paradigm have been conducted with a clinical population, as opposed to a non-clinical, collegeaged population. In a related area, no studies assessing faking-good responding in maltreating parents have examined the role of a learning bias or attitude generalization. Therefore, this current investigation attempted to expand on work across both of these research domains.

\section{Rationale for the Study and Hypotheses}

Child maltreatment continues to be a significant public health problem leading to approximately $\$ 124$ billion in costs to society due to fatalities, medical expenses, and behavioral and psychosocial problems (Fang et al., 2013; Norman et al., 2012; Runyon et al., 2004). 
Children who are exposed to maltreatment may present with a multitude of problems including risk for long-term psychological and psychiatric disorders, including posttraumatic stress, anxiety, disruptive behavior problems, and substance use/abuse, increased risk for physical health problems, poor social skills, and perhaps most troublesome, risk for abusing their own partners or children in the future (Norman et al., 2012; Runyon et al., 2004). Parenting capacity assessments (PCAs) play an integral role in determining (a) whether or not a child is removed from the offending parent's home, and (b) the parameters of what future care entails (Azar et al., 1998; Haskett et al., 1995). Data collected from PCAs inform diagnostic impressions and subsequent treatment recommendations, which are typically taken seriously by the court (Budd, 2001; Choate, 2009). A "best practice" PCA consists of a multimethod approach to data collection, often consisting of a clinical interview, self-report measure administration, parentchild behavioral observation, collateral report, and record review (Budd et al., 2011). Given the high-stakes nature of PCAs, it is imperative that useful and valid information is collected.

However, the stressful context of PCAs, for both the parent and mental health professional, as well as potential respondent and administrator bias may yield invalid data from the assessment. Self-report data is inherently at risk for bias and error given the potential difficulty of defining what constitutes parenting behaviors (e.g., what "time out" means), asking parents to reflect back on their behavior over days or months at a time, and asking sensitive questions; however, self-report measures are often used in parenting research and clinical practice (Morsbach \& Prinz, 2006). For example, self-report risk assessment measures like the Child Abuse Potential Inventory (CAPI; Milner, 1986) are typically used in PCAs, and the CAPI is the only such measure that has been normed on a child welfare population (Choate, 2009). Additionally, the CAPI has been recommended for use in PCAs because it contains validity 
scales and indices, including the Faking-good Index, to screen for socially desirable responding, a behavior that is often observed in the PCA context (Budd, 2001). Therefore, it is important to understand, from a measurement perspective, why parents may present with invalid responding, and how best to improve the self-report data gathered in these assessments, including setting up a context aimed at maximizing the parent's strengths (Azar et al., 1998).

Despite the need to understand more about the Faking-good Index on the CAPI, only three published studies in this literature review have been found to directly address which parental characteristics may be associated with faking-good responding on the CAPI (Budd et al., 2000; Carr et al., 2005; Costello \& McNeil, 2014), thus allowing for few sound conclusions to be derived. Two of these studies have taken a retrospective approach to data collection, addressing faking-good responding only after a high percentage (19-58\%) of parents had invalid profiles on the CAPI (Budd et al., 2000; Costello \& McNeil, 2014). Building upon this research base, the current study proposes four areas of consideration when studying faking-good on the CAPI. Context of assessment, cognitive functioning (i.e., intelligence and reading comprehension skills), psychopathy, and a positivity bias (i.e., the tendency to focus attention more on perceived positive information rather than negative information) have all been identified as factors which may result in a tendency for parents to fake good on the CAPI. These four areas are important to study in tandem because the "profile" of a parent (i.e., the impressions of a parent during a PCA) may be altered due to better knowledge about why this parent has an invalid profile on the CAPI. Specifically, parents with a faking-good profile on the CAPI who present with lower intellectual functioning and poorer reading comprehension skills, or who may have an unrealistically positive view of the world may have a different set of strengths and deficits than parents who are deliberately attempting to manipulate or lie. These parents may not 
necessarily be "fakers," but may be viewed as such by mental health professionals.

This study aimed to add to the existing literature by using a community sample of highrisk parents and multiple methods of evaluation. An important methodological strength in this investigation is that, consistent with the Carr et al. (2005) study, it utilized a prospective data collection, focusing on parents across a spectrum of risky parenting (i.e., parents attending parent training services, but who did not have a history of involvement in child protective services, to parents who had their children removed from the home due to possible abuse and neglect). Parents recruited for the study were receiving services at community-based mental health centers, including private practice agencies, a Child Advocacy Center, and an in-home services agency which partnered with the local Department of Health and Human Resources. Parents in this study had a range of intellectual functioning, psychopathology, and involvement with DHHR, making the sample truly "real world."

The present study had three major purposes. To begin, the present study attempted to evaluate the role of assessment context on influencing faking-good responding. Although researchers have discussed the importance of considering context during parental fitness evaluations (Carr et al., 2005; Milner \& Crouch, 1997), little research has been published which directly compared parents at varying levels of service utilization. Most studies have either focused on "normal" or "general population" parents, or parents with a documented abuse report/history. No studies have been published using parents who are "treatment-seeking" but who are not involved in the child welfare system.

Second, the investigation explored the role of four major parental characteristics (e.g., IQ, reading comprehension, self-reported psychopathy characteristics, and positivity bias) on influencing faking-good responding on the CAPI. These variables are relevant to assess because 
many of the measures that were used in the current study (e.g., CAPI, intelligence and reading comprehension testing, personality and psychopathology self-report inventories) have been cited as being frequently administered during parental fitness evaluations (Budd et al., 2011). Finally, the current study examined what most strongly predicts socially desirable responding on the CAPI. Because the CAPI is so widely used during parental fitness evaluations, these study aims attempted to inform consumers of the CAPI by (a) closing the gap in understanding which parental characteristics may influence faking-good responding, (b) attempting to differentiate "manipulative" parents versus parents who may be considered "naïve," thus potentially changing the view on a "faking" parent, and (c) using this information to help practitioners administering parental fitness evaluations to improve parental self-report (i.e., facilitating more honest responding) (Morsbach \& Prinz, 2006). If parental fitness evaluations can better predict future child maltreatment by using results that are derived from a more honest self-report, thus becoming more informative to practitioners, then family reunification can be more effectively facilitated within the legal and Child Protective Services systems. A deeper understanding of individual parental competencies can also inform needed therapy services, including, but not limited to, parent training within the context of child maltreatment.

\section{Hypotheses}

\section{Research Question 1}

Does faking good responding change by treatment context?

Hypothesis 1. Parents who are involved in the child welfare system were compared to treatment-seeking parents without a past history of child welfare involvement. It was expected that parents who are involved in the child welfare system would be more likely to have an invalid profile on the CAPI due to faking-good responding, while treatment-seeking parents 
would be more likely to have a valid profile on the CAPI.

\section{Research Question 2}

Are there differences in the intellectual functioning and reading comprehension skills between parents who fake good and those who do not?

Hypothesis 2. Parents with an elevated Faking-good index were compared to parents without an elevated Faking-good index on the CAPI (Milner, 1986). Consistent with past literature it was expected that parents with an elevated Faking-good index would have lower IQ and reading comprehension scores than parents without an elevation (Budd \& Holdsworth, 1996; Budd et al., 2000; Carr et al., 2003; Costello \& McNeil, 2014; Morsbach \& Prinz, 2006).

\section{Research Question 3}

Does parental self-reported psychopathy impact socially desirable responding on risk assessment measures?

Hypothesis 3. Parents were compared by their scores on the CAPI Faking-good Index. There appears to be no published research regarding the potential impact of psychopathy traits on faking-good responding during administration of parenting assessments. Past research has demonstrated that undergraduate students with higher levels of psychopathy, as measured by the Levenson Self-Report Psychopathy Scale (Levenson et al., 1995), and the same scale used in this study, had a greater tendency to fake good on a self-report personality inventory (Book, Holden, Starzyk, Wasylkiw, \& Edwards, 2006). However, parents in the child welfare system may be different from an undergraduate population. Given this is a relatively exploratory area in the realm of parenting, it was expected that there would be no differences between faking and nonfaking parents in regard to severity of psychopathy traits on the LSRP. If there was a difference between groups, it was expected that parents in the faking group would have more elevated 
scores on the Primary Psychopathy subscale of the LSRP than non-faking parents, indicating a greater tendency to demonstrate lying, manipulation, and superficial charm.

\section{Research Question 4}

Does parental positivity bias impact socially desirable responding on risk assessment measures?

Hypothesis 4. Parents were compared by the Faking-good Index on the CAPI. Parents' learning bias, attitude generalization, and weighting bias scores, as measured by the BeanFest experimental paradigm, were the outcome variables. It was expected that faking-good parents would (a) demonstrate a bias in learning positive information better than negative information (e.g., positive learning bias), and (b) have a greater tendency to perceive novel and ambiguous information as positive, rather than negative, demonstrated by both the attitude generalization and weighting bias variables (Costello \& McNeil, 2014).

\section{Exploratory Questions}

\section{Exploratory Question 1}

Which additional variables are significantly related to socially desirable responding on the CAPI?

Given the inconclusive results in this area of literature, no specific hypotheses were formed. The association of additional putative predictors of socially desirable responding (e.g., demographic information, parent psychopathology, and past trauma history) were examined.

\section{Exploratory Question 2}

Which variables most strongly predict socially desirable responding on the CAPI?

There are currently no studies reporting on what best predicts socially desirable responding on the CAPI, and thus a hypothesis was not created for this area. All variables that 
demonstrated significant differences between faking-good and non-faking parents, and all other variables significantly associated with the CAPI Lie Scale were used to predict the socially desirable responding. Additionally, the study's primary outcome variables (e.g., treatment setting, parental intelligence and reading comprehension, the LSRP Primary Psychopathy score, and the learning bias variable on BeanFest) were used to predict the CAPI Lie Scale.

\section{Method}

\section{Participants and Settings}

Sixty-four parent participants were recruited from mental health agencies in West Virginia and surrounding states. However, two parents only completed half of the assessment, and could not be contacted to schedule a follow-up session to complete the rest of the study. Therefore, their data were not included in study analyses, and data from 62 parents were included in the final sample. Parents were divided into a "treatment seeking without child protection involvement" group ( $n=30$ parents) and a "child protection" group $(n=32$ parents), based on (a) the agency at which they were receiving services, and (b) whether or not they endorsed a history of involvement in child protective services for either themselves or their child on the study's demographics form. Given the prospective nature of the study, child protection parents were not necessarily required to have a founded report of abuse or neglect and/or did not have to be involved with Department of Health and Human Resources (DHHR) services due exclusively to physical abuse. Rather, the family must have had some type of child protection involvement. This means that either the parent or the child must have been in contact with DHHR due to a documented child abuse report or a history of concerning behaviors by DHHR to place the child "at risk" for future harm.

It should be noted that this study did not include a "normal" control group (i.e., a sample 
of parents from the community with no involvement in parenting services and/or DHHR). Although the CAPI has been used with normal controls in the past (see Milner, 1986; Milner \& Crouch, 1997), studies specifically investigating which parental characteristics differentiate faking-good and non-faking groups have focused on an at-risk (i.e., parents who either were undergoing a PCA, or who were involved in therapy services due to risky parenting behaviors) sample (Budd et al., 2000; Carr et al., 2005; Costello \& McNeil, 2014). Additionally, although Milner, (1986) reported that about 7-11\% of normal control parents fake good on the CAPI, studies with a clinical population have yielded higher percentages of faking-good profiles (19$58 \%$ ), and have thus been able to more easily compare a faking and non-faking group. Given the major purpose of this study was to study faking profiles (as opposed to comparing normal controls to at-risk parents), study recruitment focused its efforts on including at-risk parents. Specifically, parents (regardless of treatment context) were compared by their Faking-good index on the CAPI, and were grouped by either an elevated Faking-good index (e.g., a score of 7 or 8 , based on level of education on the CAPI Lie Scale, and a Random Response scale below cutoff), or an index within normal limits.

Child protection parents were recruited from community mental health centers located in West Virginia or surrounding states. Consistent with parent inclusion criteria, agencies used for recruitment of the child protection parents were required to have some involvement with the child welfare system or child protective services organization in their county. Inclusion criteria for child protection parents included the following: (a) the parent must have been involved in an agency which partners with DHHR, (b) the parent must have been involved in the child welfare system either directly or indirectly (i.e., parent may have been a non-offending caregiver, but child was receiving services), (c) the parent must have had at least one child under the age of 18 
at the time of the study, and (d) the parent must have been willing to complete the assessment battery.

Treatment-seeking parents were recruited from mental health agencies in and around Morgantown, West Virginia. Inclusion criteria for treatment-seeking parents were as follows: (a) the parent must have been receiving parenting services (e.g., behavioral parent training), (b) the parent must have denied having a previous report of abuse, neglect, or involvement in the child welfare system, (c) the parent must have had at least one child under the age of 18 at the time of the study, and (d) the parent must have been willing to complete the assessment battery. Exclusion criteria for all parents included the following: (a) the parent was under 18 years of age at the time of the study, and (b) the parent could not speak English. No parents were excluded from participating in this study due to the above criteria. The percentage of parents who refused to complete the permission to contact flyer, or who later refused to be scheduled for an assessment was not measured, and thus there are no data available on refusal rates of participation.

\section{Procedure}

The Institutional Review Board of West Virginia University reviewed and approved this proposal and informed consent prior to recruitment. Recruitment began in July 2012, and was completed in January 2014. All data were collected by either Ms. Amanda Costello (first author of this study), or Ms. Nancy Wallace, both who were trained and supervised graduate student researchers. Both Ms. Costello and Ms. Wallace received assessment training through coursework offered through the Department of Psychology at West Virginia University. This training prepared both individuals to administer the self-report questionnaires, as well as the IQ and reading comprehension subtests. Additionally, Ms. Costello trained Ms. Wallace in the 
implementation of study procedures before Ms. Wallace began data collection. Training consisted of an overview of the assessment battery, and then a discussion of each step of the procedure. Ms. Wallace was then asked to role-play administering the study's assessment battery to Ms. Costello. Ms. Costello was also available for consultation throughout data collection. Ms. Costello initially recruited therapists from five community-based and universitybased agencies to participate in the study, and Ms. Wallace recruited therapists from an additional agency toward the end of data collection. The six agencies included a county child advocacy center, an in-home service provider, which contracted with the Department of Health and Human Resources in Monongalia and Marion counties in West Virginia, the Quin Curtis Center at West Virginia University, two community-based private practices and an outpatient mental health clinic. Prior to participant recruitment, all community therapists were given recruitment materials (e.g., permission to contact form) and were given a brief presentation regarding the basic premise of the study, as well as the expectations of the therapists in recruitment and parents completing the study. After therapists were made aware of the study procedures and expectations, they were asked to present a permission to contact form to their clients. The permission to contact form included a brief introduction to the study and its basic aims, the contact information for the research team, and an area in which parents could leave their contact information to be recruited for the study. Appendix A contains a sample permission to contact form. This form acted as means of contacting an interested parent; no study screening was conducted at the time of distribution. Interested parents left their contact information on the form, which was delivered back to the research team. The parent was then contacted, either in person or over the phone, to determine study eligibility and to schedule an assessment. Assessments either occurred in the community mental health agency where the parent 
and/or child were receiving services or in the parent's home. The decision regarding location was based on the parent's discretion. Overall, the majority of parent participants $(66.1 \%)$ completed the assessment in their home. Ms. Costello traveled with the service provider to parents' homes who were receiving services through the in-home agency affiliated with DHHR. All other assessments took place only with Ms. Costello or Ms. Wallace, and an undergraduate research assistant (when possible).

Before administering the assessment battery, Ms. Costello or Ms. Wallace read the study's informed consent document to the parent. The parent then provided written consent to participate in the assessment, and received a copy for their records. No assessment measures were given prior to obtaining informed consent. After consenting for the study, parents were administered a battery of assessments. The order of assessments remained the same for all participants. All participants read and answered the self-report measures themselves; no participants required the measures to be read to them. To protect the privacy of parents involved in this study, all individuals received an identification number for their assessment battery, and no identifying information was recorded on the measures. The list matching parent names to identification numbers was stored separately and securely from the assessments.

The presentation of assessment measures was as follows: the Child Abuse Potential Inventory (Milner, 1986), the Marlowe-Crowne Social Desirability Scale (Crowne \& Marlowe, 1960), the simplified version of the BeanFest game (Fazio et al., 2004; Pietri et al., 2012; see below for detailed description as to what the task entailed), the two-subtest version of the Wechsler Abbreviated Scale of Intelligence, Second Edition (vocabulary and matrix reasoning subtests; Wechsler, 2011), the reading comprehension subtest of the Wechsler Individual Achievement Test - Third Edition (Wechsler, 2009), the Levenson Self-Report of Psychopathy 
(Levenson et al., 1995), the Beck Depression Inventory-II (Beck et al., 1996), and a demographics form. Appendix B indicates the presentation of the assessment battery to each parent. The demographics form included sensitive information (e.g., information regarding the parent's own trauma history, as well as his/her involvement in DHHR), and thus, was placed at the end of the assessment battery to decrease potential respondent bias. Parents typically completed the assessment battery in 90-120 minutes. Upon completion of the measures, parents were compensated for their participation with a $\$ 30.00$ gift card to WalMart, and were given the opportunity to ask questions about the study procedures.

\section{Behavioral Task}

Parents were asked to complete the simplified version of the BeanFest game (Fazio et al., 2004; Pietri et al., 2012) during the assessment. The BeanFest software was loaded previously onto a Toshiba Satellite M505-S4940 laptop computer owned by Ms. Costello, or a Dell Latitude E6500 computer owned by West Virginia University, and all data from the game were automatically entered into the computer using the Inquisit software program (Inquisit 3.0.6.0). Data were then transferred to an Excel file for cleaning and coding, and a finalized BeanFest dataset was entered into SPSS for study analyses. Parents were asked to use the laptop keyboard and computer to complete the presented task.

Three individuals (Ms. Costello, Ms. Wallace, and Ms. Reeva Morton, an undergraduate student) administered BeanFest to participants. Ms. Costello was initially trained in administering the BeanFest by Natalie Shook, Ph.D., who has extensive training and research experience in the BeanFest. Ms. Costello then trained Ms. Wallace and Ms. Morton in how to administer the task. Training involved showing each individual how to read instructions for the task, and having each individual "role play" the task with Ms. Costello. Additionally, Ms. 
Costello, Ms. Wallace, and Ms. Morton were all trained to ignore questions, and withhold attention during the task. Ms. Costello administered BeanFest to 36 (58\%) participants, Ms. Wallace administered the BeanFest to 14 (22.6\%) participants, and Ms. Morton administered BeanFest to $12(19.4 \%)$ participants. To check to see if who administered the BeanFest affected performance, scores obtained on the BeanFest when administered by Ms. Costello were compared to scores obtained on the BeanFest when administered by Ms. Wallace and Ms. Morton (these scores were combined to create one variable). Independent-samples $t$-tests revealed no significant differences on how accurately participants learned positive beans or negative beans across Blocks 1-3 in the game phase. There were also no differences between administrators on how many games participants won, $t(57)=-.37, p>.05$ or lost, $t(57)=.04, p$ $>.05$. Additionally, in the test phase, there were no significant differences on how well participants learned negative beans, $t(57)=-1.04, p>.05$ or positive beans, $t(57)=-1.21, p>$ .05. Therefore, performance on the BeanFest was not found to be confounded by who administered the task.

Before beginning the task, parents were instructed to read directions for BeanFest on the computer screen, while also listening to the same directions read orally by Ms. Costello, Ms. Wallace, or Ms. Morton (this depended on who administered BeanFest). Appendix C contains a copy of the directions. The directions used in this study were slightly modified by Ms. Costello to include simpler language, which was thought to be more appropriate with a community sample. Modifications are noted in bold and italicized script. After the directions to BeanFest were administered, parents had the opportunity to ask questions about the game, but were notified that they could not ask any other questions once the game commenced. Consistent with past studies, parents began the task by completing a "practice phase" of four trials (Shook et al., 
2007).

Once the practice phase was complete, parents then began the actual BeanFest game, beginning with the "game phase" (Shook et al., 2007). In this study the game phase marked the beginning of data collection and consisted of 3 blocks of 40 trials. Each bean was randomly presented on each trial. Parents began the game phase with a total score of 50 points, and then were tasked with learning which beans were "positive" (i.e., added 10 points to their score) and which beans were "negative" (i.e., subtracted 10 points from their score). Point totals only changed if the parent chose to accept the bean; if parents avoided the bean, they did not incur a point loss. The current study used a full-feedback version of BeanFest, in which parents were shown information about each bean (i.e., the true point value of the bean, and the effect that bean had or would have had on their score) regardless if they decided to approach or avoid the bean. If parents won (reached 100 points) or lost (reached 0 points) a game, they were notified they must keep playing until the game phase was complete. Therefore, the BeanFest game would restart, beginning at 50 points, each time the parent won or lost the game. When this occurred, participants were given the same set of beans, so they did not have to learn new beans during each block.

Once completed with the game phase, parent participants moved to the "test" phase (Shook et al., 2007). Before beginning the test phase, the individual administering BeanFest (Ms. Costello, Ms. Wallace, or Ms. Morton) paused the game and presented additional directions about the phase. The test phase consisted of 100 beans, which included the 40 game beans, and 60 novel beans. During this phase parents were asked to label whether beans presented are "helpful" or "harmful." No point totals or feedback were given at this time. Once the parents completed the test phase, they were finished with BeanFest. No debriefing script was used, and 
parents moved on to the next phase of the assessment.

\section{Measures}

Demographic information. (Appendix D). A demographic form was constructed for this study, and included the following information: parent age and gender, parent socioeconomic status as measured by parental occupation, total family income, and level of education. The parent's own abuse/trauma history was recorded quantitatively (i.e., as present/absent) and qualitatively (i.e., explaining the nature of the abuse/trauma). Quantitative and qualitative information were also collected regarding the parent's involvement in the child welfare system, and items tapping this domain included the following information: number of reports (either for the parent or his/her child), the year of each report, a description of each report, and outcome of each report.

Socially desirable responding. The Child Abuse Potential Inventory-IV (CAPI; Milner, 1986) was administered to determine socially desirable responding in this study. The CAPI is a 160-item self-report measure used as a screening device for physically abusive and non-abusive parents. It contains six abuse risk factors including: distress, unhappiness, rigidity, problems with child/self, problems from family, and problems from others. It also contains a broadband dimension for identifying child abuse. There are two possible clinical cutoffs for the abuse risk score on the CAPI; a score of 166 is considered the "signal detection" cutoff (i.e., reducing the number of false negatives and false positives), and a score of 215 is considered the more conservative cutoff. Both have been used in research and clinical studies; however, Milner, 1986 suggests using the more conservative cutoff score of 215 , to reduce the rate of false positive classifications. The measure also contains three validity scales, including lie (containing 18 items), inconsistency and random responding. Combinations of the three validity scales identify 
additional response distortions including the Faking-good Index, the Faking-bad Index, and the Random response Index. The Faking-good Index contains an elevated lie scale (either 7 or 8 based on parental reading level) and a Random Responding scale below a cutoff of 5. The Faking-bad Index contains an elevated Random Responding scale and an inconsistency scale within normal limits. The Random Response Index contains both an elevated Random Responding scale and Inconsistency scale. Internal consistency on the CAPI is strong and ranges from .95 to .86 (Milner, 1986). The CAPI also has high test-retest reliability at a one-day interval (.91) to a three-month interval (.75). The present study used the Faking-good Index as the primary dependent variable.

The Marlowe-Crowne Social Desirability Scale (MCSDS; Crowne \& Marlowe, 1960) was used as a complementary measure to assess socially desirable responding. The MCSDS is a 33-item scale utilizing a forced-choice "true/false" format used to assess behaviors that may be socially acceptable but unlikely to occur (Crowne \& Marlowe, 1960). Items on the measure include, "I never hesitate to go out of my way to help someone in trouble" and "There have been times when I was quite jealous of the good fortune of others" (see Crowne \& Marlowe, 1960). It has demonstrated high internal consistency (.88) and test-retest reliability (.89). The MCSDS has shown associations with the MMPI (Hathaway \& McKinley, 1943) validity, clinical and derived scales (Crowne \& Marlowe, 1960). The present study used the MCSDS total score. Higher scores on the MCSDS indicate higher levels of socially desirable responding

Intelligence. The Wechsler Abbreviated Scale of Intelligence, Second Edition (WASI-II; Wechsler, 2011) was used to measure parental intelligence. The WASI-II was developed as a screening device which contains four subtests (Vocabulary, Block Design, Similarities, Matrix Reasoning; Wechsler, 2011), and can be administered to individuals aged 6 through 90 years old. 
It measures fluid and crystallized intelligence and yields a Full Scale IQ (Wechsler, 2011). It can be administered by either a bachelor's or master's level clinician in either a four- or two-subtest version, and must be interpreted by a master's- or doctorate- level clinician. The WASI-II has demonstrated strong internal consistency $(r=.90-.97)$, test-retest reliability $(r=.90-.96)$, and interrater reliability ( $r=.94-.99)$. Additionally, the WASI-II has demonstrated a strong internal structure, along with the Verbal Comprehension Index and Perceptual Reasoning Index each measuring a unique construct. The WASI-II has also demonstrated strong associations with the original WASI, and other Wechsler tests, indicating excellent concurrent validity (Wechsler, 2011). The primary investigator and another graduate researcher administered the WASI-II to participants. Both had sufficient training in psychological test administration and interpretation (e.g., were enrolled in a graduate course in assessment, and had supervised experience in using the measure; Wechsler, 2011) before the onset of the study. The present study used the twosubtest version of the WASI-II to calculate the Full Scale IQ, which was used in analyses. Specifically, the Full Scale IQ was calculated by taking raw score data from the Vocabulary and Matrix Reasoning subtests, converting these data to standard scores, and deriving the full scale IQ from a combination of the standard scores.

Reading comprehension. The WIAT-III reading comprehension subtest (Wechsler, 2009) was administered to parents. The WIAT-III can be individually administered via pencilpaper format to subjects ages 4 years 0 months to 50 years 11 months. Although the WIAT-III has been normed on this age range, four participants in the current study were older than 50 years 11 months (age range: 54-68 years old). Thus, data from these participants may be slightly confounded because the WIAT-III does not have norms for this age range. It demonstrates strong split-half reliability for subtests $(r=.83-.97)$ and test-retest reliability. Additionally, it has been 
normed on approximately 3,000 students and adults. The reading comprehension subtest of the WIAT-III measures literal and inferential comprehension and tests individuals by using visual, auditory, and visual-auditory stimuli. The WIAT-III was also administered by the primary investigator and graduate researcher, both of whom had sufficient training in psychological assessment administration and interpretation at the onset of the study (see description above in WASI-II section). The present study used the reading comprehension subscale standard score. This score was calculated by taking the raw score of the reading comprehension subscale and deriving a weighted raw score based on age and level of education. The weighted raw score was then converted to the standard score, based on parent age.

Psychopathy. The Levenson Self-Report Psychopathy Scale (LSRP; Levenson et al., 1995) was administered to parents to assess behaviors associated with psychopathy. The LSRP is a 26-item measure, initially developed for use in non-forensic settings, using a 1 (strongly disagree) to 4 (strongly agree) Likert scale, with higher scores indicating higher levels of psychopathy. In the present study an adapted LSRP using a 5-point Likert scale, but with the same 33 items was used. Thus, means derived from this sample may be slightly different than means derived from other samples using the LSRP. The LSRP yields two domains measuring primary (i.e., selfish, callous, manipulative traits) and secondary (i.e., impulsivity, poor behavioral control) psychopathy (Book, Quinsey, \& Langford, 2007). The primary and secondary psychopathy domains on the LSRP map onto Factors 1 and 2 or the Psychopathy Checklist - Revised (Hare, 1991), respectively (Book et al., 2007). The measure has demonstrated strong internal consistency (alpha $=.83)$, test-retest reliability $(r=.83)$, and good criterion and construct validity (Lynam et al., 1999). Domain scores on the LSRP have been validated for use in non-offending, undergraduate, as well as offending populations, and have 
been associated with psychopathy in a forensic sample (Brinkley. Schmitt, Smith, \& Newman, 2001; Epstein, Poythress, \& Brandon, 2006; Lynam et al., 1999; Parrott \& Zeichner, 2006; Reidy, Zeichner, \& Martinez, 2008). The present study used the Primary Psychopathy, Secondary Psychopathy, and total score on the LSRP. The total score was derived from summing scores from the primary and secondary psychopathy domains on the LSRP. Higher scores indicate higher levels of psychopathic personality traits.

Positivity bias. The simplified version of BeanFest Game (Fazio et al., 2004; Pietri et al., 2012) was administered to parents to test for the presence of a learning and attitude generalization bias. In this study, the presence of a positivity bias was investigated. Specifically, participants were tested to assess if there were individual differences related to the extent to which individuals learned positive information in BeanFest to a greater degree than negative information. Additionally, the attitude generalization and weighting bias measured the degree to which parents classified novel beans as either positive or negative.

BeanFest is a performance-based measure developed to assess attitude formation to novel stimuli. It exposes participants to these novel stimuli referred to as "beans" which vary by shape and number of speckles (Fazio et al., 2004), and developers of BeanFest created a 10x10 matrix of beans to be used in the paradigm. The beans vary by valence (either positive or negative), and participants who are administered BeanFest are required to learn to either approach "positive" (i.e., beans which add points to the participant's total score) or avoid "negative" (i.e., beans which subtract points to the participant's total score) beans. Participants begin each trial with a total score of 50, and gain or lose 10 points based on the bean's valence. For participants, the goal in BeanFest is to determine which beans (based on shape and number of speckles) are "good" beans (i.e., add 10 points to the total score), and which are "bad" beans (i.e., subtract 10 
points from the total score).

Participants are administered practice, learning, and testing trials. In the learning phase at the beginning of each trial the participant is shown one bean and must determine whether to approach or avoid the bean. In the full-feedback version of BeanFest, the participant's "energy level" (i.e., total score; see Fazio et al., 2004) is always visible. After either approaching or avoiding the bean, feedback is given to the participant as to the value of the bean. The "effect of the bean" and the "net gain/loss" of the bean are displayed (Fazio et al., 2004) during feedback sessions. Each trial ends with feedback to the participant. In this study participants received the full-feedback version of BeanFest.

Because a community sample (over a college student sample), with potentially more heterogeneity in IQ and level of education, was recruited for this study, the simplified version of BeanFest was utilized. The simplified version of BeanFest differs slightly from the original version, in that the game bean stimuli are taken from the four corners of the $10 \times 10$ matrix, thus producing the clearest distinction between "good" and "bad" stimuli. Specifically, ten beans were taken from each corner, and each corner was assigned either a positive (i.e., +10$)$ or negative (i.e., -10) value (Pietri et al., 2012). The simplified version was chosen to increase the likelihood that the recruited sample would actually be able to learn the difference between good and bad beans.

Three variables from BeanFest were examined in this study. All were calculated from data from the test phase. The first variable, learning bias, was created by calculating a difference score between the proportion of positive beans learned correctly and proportion of negative beans learned correctly. In the test phase, participants were exposed to the 40 game beans from the game phase, as well as 60 novel beans. The learning bias variable was calculated by taking 
valence scores (e.g., positive or negative) from the participant on the 40 game beans. The valence score from the participant was deemed either accurate or inaccurate, based on the true valence score of the bean. Then, proportion of positive beans learned and proportion negative beans learned were calculated, and a difference score between both variables was created. A positive difference score indicated presence of a positivity bias (i.e., participants learned positive beans better than negative beans during the test phase). By contrast, a negative difference score indicated presence of a negativity bias (i.e., participants learned negative beans better than positive beans). A difference score of zero indicated no learning bias.

The second variable, attitude generalization, was created by taking valence ratings from all 60 novel beans to which participants were exposed in the test phase. The attitude generalization variable measured the tendency for participants to classify new information as either positive or negative. During the test phase, individuals either classified beans as positive or negative, which were then coded into 1 (positive) or -1 (negative). Participants' valence responses were then averaged across the 60 trials to indicate attitude generalization. A positive score on this variable indicated a tendency to generalize positive information over negative information, while a negative score on this variable indicated the opposite.

Additionally, a weighting bias variable was created for the study, following outlines from Pietri et al. (2012). Scores from each trial of twenty-four "ambivalent" beans (see Pietri et al., 2012 for information on how these ambivalent beans were determined) were averaged to create the weighting bias variable. Similar to the attitude generalization variable, these ambivalent beans were taken from each quadrant of the complete 10x10 matrix, and did not strongly resemble either positive or negative beans. Participants were not previously exposed to these beans in the game phase, and thus they were considered completely novel beans. Because these 
beans were considered ambiguous, or ambivalent, they were determined to best represent a true weighting bias (i.e., the tendency to more strongly weight novel information as more negatively or positively).

Depressive symptoms. The Beck Depression Inventory-II (BDI-II; Beck et al., 1996) was administered to parents to assess depressive symptoms. The BDI-II is a 21-item self-report measure developed to correspond with the diagnostic criteria for depressive disorders. It contains items which measure behavioral, cognitive, and physiological components of depression, and participants respond to individual items using a 0 (symptoms not present) to 3 (high intensity/severity of symptoms present) Likert scale. The BDI-II yields a total score (0-63), based on the raw participant data, which rates minimal through severe depressive symptoms. Specifically, a total score of 0-9 on the BDI indicates minimal symptoms, a total score of 10-18 indicates mild symptoms, a total score of 19-29 indicates moderate symptoms, and a total score of 30-63 indicates severe symptoms (Beck et al., 1996). The BDI-II has demonstrated strong internal consistency in an outpatient sample (.93) as well as strong content and construct validity. The present study used the BDI-II total score.

\section{Data Analytic Plan}

\section{Preliminary Analyses}

Each variable was analyzed for missing data; if more than $10 \%$ of data were missing on a measure, then appropriate steps were taken to account for missingness. Normality of data was also assessed, by examining skewness, kurtosis, homogeneity of variance, and independence of observations. Overall, three participants had over $10 \%$ of their data missing on the BeanFest trials, and how these data were handled is described below. Two participants had over $10 \%$ of data missing on the Marlowe Crowne Social Desirability Scale (Crowne \& Marlowe, 1960), 
which was due to administrator error (e.g., pages were missing from the measure when it was given to the participants). Scores from these two MCSDS profiles were not used in subsequent analyses. No other participants had more than $10 \%$ missing data on any other measure. Data that violated normality were corrected. If relevant, the correction process is described below.

\section{Descriptive Analyses}

This study also collected descriptive data using demographic information derived from parents. Data were collected for all parents regarding number of mothers and fathers, mean age of mothers and fathers, frequency of race and ethnicity for parents, and frequency of caregiver education, income status, and marital history. Data were also collected regarding whether or not the parent had a trauma history. Additionally, data were collected regarding number of child protective sevices reports (either for the parent or his/her child), the year of each report, a description of each report, and outcome of each report. Parents in the faking and non-faking groups were compared by demographic to assess for any differences in demographic variables (e.g., level of education, income, age, and trauma history).

\section{Primary Analyses}

To test study objectives, either chi-square (for categorical variables) or independent samples $t$-tests (for continuous variables) were conducted comparing parents grouped by the presence or absence of an elevated Faking-good Index on the CAPI (dichotomous "yes/no" variable). To test exploratory objectives, a correlational matrix using the CAPI Lie Scale (continuous variable) was conducted, and included both categorical and continuous variables. Two stepwise multiple regressions were then conducted. In the first regression model, variables that were significantly associated with the CAPI Lie Scale were entered into the analysis. In the second regression model, only the primary outcome variables thought to be most critically 
related to the CAPI Lie Scale were entered into the analysis. These variables included the treatment setting (i.e., child protection parents versus treatment-seeking parents without child protection involvement), scores on the WASI-II and WIAT-II, the Primary Psychopathy score on the LSRP, and the learning bias score on the BeanFest.

A multiple regression was determined to be appropriate (over a binary logistic regression), because there were unequal numbers of parents in the faking (22 parents) and nonfaking (40 parents) groups. Additionally, given there appear to be no published studies examining which parental characteristics best predict faking-good responding, a stepwise method was chosen, based on the exploratory, atheoretical nature of this research question. Results from these analyses are described in further detail below.

\section{Results}

\section{Demographic Characteristics of the Sample}

Thirty treatment-seeking without child protection parents and 32 child protection parents participated in the study. The majority of participants were female (85.5\%), Caucasian (93.5\%), and had an average age of $36.32(S D=10.45)$. The majority of parents were residents of Monongalia County, West Virginia (66.1\%), although a total of seven counties from West Virginia and Pennsylvania were represented in the study. Parents were most likely to have a high school education (41.9\%), be unemployed (41.9\%), and have a household income of less than $\$ 20,000$ at the time of the study (37.1\%). The majority of parents were not married $(54.8 \%)$. Parents in the study demonstrated average intellectual functioning $(M=97.43, S D=10.45)$ but scores ranged from a low of 59 to a high of 134 on the WASI-II. Additionally, over half of the participants $(51.6 \%)$ had experienced some form of abuse or trauma prior to the start of the study. Qualitative analyses revealed type of trauma varied, and included experiencing domestic 
violence, sexual, verbal, and physical assault, neglect, traumatic grief and loss of a loved one, car accident, and serious physical health problems. In the overall sample parents had an abuse risk score of $124.20(S D=88.90)$ which is under the signal detection clinical cutoff of 166 (and more conservative cutoff of 215) on the CAPI. However, across all demographic variables, the participants recruited in this study exemplified a true "real-world" and "at-risk" sample at the time of study completion. Table 1 provides more detailed information on the demographic characteristics of the entire sample.

Demographic information was also compared across the faking and non-faking groups, using independent-samples $t$-tests for continuous variables and chi-square tests for categorical variables. In this study $22(35.5 \%)$ parents had an invalid profile on the CAPI due to faking-good responding. No parents had an invalid profile due to violations of the Faking-bad or Inconsistency Indices on the CAPI. As previously noted, to have an elevated Faking-good Index a respondent must have an elevated Lie Scale score (7 or 8 and above based on level of education) and a Random Responding score below the clinical cutoff (5 or less). In the fakinggood group, parents had a mean Lie Scale score of $9.50(S D=1.73$; range $=7-13)$, and a mean of 2.09 on the Random Response Scale $(S D=1.15$; range $=0-4)$. In the non-faking group, parents had a mean Lie Scale score of $4.07(S D=2.17$; range $=0-7)$ and a mean of 2.05 on the Random Response Scale $(S D=1.81$; range $=0-10)$. Parents in the faking-good group had an average age of $36.54(S D=11.13)$, were female $(95.5 \%)$ and Caucasian $(95.5 \%)$. The majority of individuals was married at the time of the study (54.5\%), had a high school education $(45.5 \%)$ and was employed full-time (54.5\%). However, parents in the faking-good group typically had an income level at $\$ 20,000$ or below $(40.9 \%)$. Forty-five percent of the faking-good group experienced some form of trauma in their life. Table 2 reflects demographic information broken down into 
the faking and non-faking groups of parents. Overall, parents in the faking-good and non-faking groups did not significantly differ by their basic demographic information.

\section{BeanFest Data}

Missing data/response bias analysis. In the game phase, one participant demonstrated a response bias, in which all beans from blocks 2 and 3 were avoided. Due to this bias, the data were considered an invalid measure of learning, and thus this participant's data were not included in subsequent analyses. Additionally, one participant had $47 \%$ of data missing on Block 2, and one participant had $65 \%$ of data missing on Block 1 . Analyses were conducted comparing game phase learning with and without the inclusion of these trials. There were no differences when the trials were not included, and so due to the large amount of missing data on these trials, they were removed from the final game phase analyses.

In the test phase, one participant engaged in response bias, reporting $96 \%$ of the beans as negative (this was the same participant who demonstrated a response bias during the game phase). Again, due to the invalid data, this participant's data were not included in subsequent analyses. Additionally, one participant had $16 \%$ of data missing, and one participant had $38 \%$ of data missing. To note, these were the same participants with the high amount of missing data in the game phase. Consistent with the game phase, analyses were run with and without data from these two participants. There were no differences when data from these participants were not included, and so due to the potentially invalid information, these scores were removed from the final test phase analyses. Visual inspection of each of the three participants whose data were not included in game and test phase analyses indicated that all individuals had an IQ score of less than 75 on the WASI-II. Therefore, the three participants with the high amount of missing data may have had invalid responding due to low intellectual functioning. 
Game phase learning. A 2 (valence) x 3 (block) repeated measures analysis of variance was conducted to assess approach behavior. Valence was measured by either positive or negative beans. There was a significant main effect for valence, $F(1,58)=200.47, p<.001$, $\eta^{2}$ partial $=$ .76 , and participants approached a greater proportion of positive beans $(M=.85)$ than negative beans $(M=.22)$. Mauchley's test for sphercity was violated for the main effect of block, $\chi^{2}(2)=$ $9.00, p<.05$, and thus degrees of freedom were corrected using the Greenhouse-Geisser estimates of sphercity $(\varepsilon=.87)$. However, there was not a significant main effect for block, $F$ $(1.74,101.20)=2.37, p>.05, \eta^{2}$ partial $=.03$. Mauchley's test for sphercity was violated for the interaction of block and valence, $\chi^{2}(2)=15.01, p<.001$, and thus degrees of freedom were corrected using the Greenhouse-Geisser estimates of sphercity $(\varepsilon=.81)$. There was a significant interaction effect between valence and block, $F(1.62,94.18)=36.76, p<.001, \eta^{2}$ partial $=.38$. This indicates that valence of bean had different effects on approach behavior across the three blocks in the game phase. Contrasts revealed that a greater proportion of positive beans was approached across Blocks $1(.79), 2(.86)$, and $3(.90), \eta^{2}$ partial $=.28$, and a greater proportion of negative beans was avoided across Blocks $1(.31), 2(.18)$, and $3(.15), \eta^{2}$ partial $=.27$. Figure 1 demonstrates the main effect of valence and interaction effect of valence and block in participant approach behavior in the game phase.

Additionally, a 2 (valence) x 3 (block) repeated measures analysis of variance was conducted to assess participant accuracy over time. Consistent with the previous analysis, valence was measured by either positive or negative beans. There was a significant main effect of valence, $F(1,58)=6.87, p<.05, \eta^{2}$ partial $=.10$, and participants demonstrated correctly learning a larger proportion of positive beans $(M=.85)$ beans over negative beans $(M=.77)$; however learning was high for both positive and negative beans. Mauchly's test for sphercity 
was violated for the main effect of block, $\chi^{2}(2)=16.61, p<.001$, and thus degrees of freedom were corrected using the Greenhouse-Geisser estimates of sphercity $(\varepsilon=.79)$. There was a significant main effect of block, $F(1.59,92.59)=36.68, p<.001, \eta^{2}$ partial $=.45$. Pairwise comparisons revealed that accuracy improved over time. Proportion scores indicated that, on average, there was a .73 accuracy rate in Block 1, a .83 accuracy rate in Block 2, and a .89 accuracy rate in Block 3. Mauchly's test for sphercity was violated for the block x valence interaction term, $\chi^{2}(2)=8.06, p<.001$, and thus degrees of freedom were corrected using the Greenhouse-Geisser estimates of sphercity $(\varepsilon=.88)$. However, there was not a significant interaction effect between valence and block, $F(1.76,100.73)=2.23, p>.05, \eta^{2}$ partial $=.05$. Figure 2 demonstrates the main effects for both valence and block in participant accuracy during the game phase. Overall, participants demonstrated greater accuracy learning positive over negative beans and greater learning across the three game phase blocks in BeanFest. This high level of learning suggests that the simplified version of BeanFest is able to be used with a community sample (as compared to a college-aged sample) with varying levels of IQ and education.

Test phase learning. Overall, participants in this study demonstrated learning a high proportion of both positive $(M=.89, S D=.16)$ and negative beans $(M=.85, S D=.22)$ in the test phase. One-sample $t$-tests were conducted to assess whether or not participants learned the positive and negative beans above chance. Participants correctly identified both positive, $t(58)=$ 18.93, $p<.001$ and negative, $t(58)=11.97, p<.001$ beans well above chance. These results, therefore, support the use of the simplified version of BeanFest in a community sample, of individuals with varying levels of intelligence and education. Additionally, a one-sample $t$-test was conducted to assess whether or not a learning bias was actually present (i.e., compared 
learning bias variable to zero). Results indicated that, on average, there was not a learning bias present in this sample, $t(58)=1.62, p=.10$. These results indicate that participants in this sample may have "overlearned" the beans, to the point where learning may have been "maxed out," and a specific learning bias could be parsed out. The overlearning of information during the test phase may have been affected by participants completing the simplified version of the BeanFest game in this study. Additionally, although there was a range of scores on the WASI-II, the sample presented with average intelligence $(M=97.43, S D=10.45)$, and the majority of individuals had either a high school $(41.9 \%)$ or college $(37.1 \%)$ education. Therefore, the standard version of the BeanFest, originally used by Fazio et al. (2004), may have been more appropriate for the parents in this study.

Similar to the learning bias variable, a one-sample $t$-test was also conducted to assess whether or not there was a negative or positive attitude generalization and weighting bias greater than zero. Results indicated that, on average, there was not a positive or a negative attitude generalization observed in this sample, $t(58)=.60, p=.54$; there was also not a discrete positive or negative weighting bias, $t(58)=-.41, p=.67$. The lack of a learning generalization or weighting bias in this study is inconsistent with what has been observed in past studies utilizing BeanFest (Fazio et al., 2004; Pietri et al., 2012; 2013; Shook et al., 2007). On average, participants in these studies typically demonstrated a negative attitude generalization and/or weighting bias, although in some studies there has been considerable variability around the mean (Pietri et al., 2012).

One possible explanation for the lack of a true positive or negative attitude generalization and weighting bias in this sample is that there was a split between individuals who demonstrated either a positive or negative bias, which may have "washed out" the overall effect when averaged 
across the whole sample. Further inspection of the data indicated that $31(50.0 \%)$ of participants demonstrated an attitude generalization favoring positive stimuli, while $28(45.5 \%)$ participants demonstrated the opposite (e.g., negative attitude generalization). Additionally, 28 participants (45.2\%) demonstrated a weighting bias favoring positive stimuli, and 28 participants (45.2\%) demonstrated a weighting bias favoring negative stimuli. Three $(4.8 \%)$ participants did not demonstrate a weighting bias favoring either positive or negative stimuli (i.e., weighting bias variable was zero). Therefore, this equal split between participants most likely would have canceled out an overall effect for the attitude generalization and weighting bias variables when averaged across the entire study. Table 3 provides overall means for all BeanFest game and test phase variables.

Association of BeanFest variables with demographic information. A correlational matrix, containing both categorical and continuous variables was created to examine potential associations between BeanFest game and test variables and relevant demographic information (e.g., age of participant, WASI-II scores, and BDI-II scores) from participants in the study. For simplicity, the approach and accuracy variables constructed from the game phase were aggregated across Blocks 1-3 into one "approach" variable. Table 4 presents this matrix. Overall, learning on the game phase (as measured by approach behavior and accuracy) was associated with scores on the WASI-II, but not participants' age or BDI-II scores. Approach and accuracy scores for positive beans were positively correlated with WASI-II scores, indicating that higher IQ scores was associated with greater accuracy and approach of positive beans. Approach scores were negatively correlated with WASI-II scores, and by contrast, accuracy scores were positively correlated with WASI-II scores. This indicated that higher IQ scores were associated with greater avoidance of negative beans, and thus increased accuracy. 


\section{Primary Analyses}

\section{Research question 1. Does faking good responding change by treatment context?}

To test this question, a chi-square analysis was conducted, comparing faking versus nonfaking parents (coded as a dichotomous variable) by treatment context (also coded as a dichotomous variable). There was not a significant difference between groups on treatment context, $\chi^{2}(1, N=62)=.51, p=.47$, Cramer's $\mathrm{V}=.09$ (no effect). In the faking-good group, ten parents $(45.5 \%)$ came from the child protection group, and 12 parents $(54.5 \%)$ came from the treatment-seeking without child protection group.

\section{Research question 2. Are there differences in the intellectual functioning and} reading comprehension skills between parents who fake good and those who do not?

An independent-samples $t$-test was used to compare faking versus non-faking parents by the WASI-II total score. The WASI-II data met all assumptions of normality, including following a normal curve. Therefore, no transformations were required. Parents in the faking group $(M=93.45, S D=16.10)$ did not significantly differ from parents in the non-faking group $(M=99.62, S D=18.92), t(60)=-1.29 p=.21, \eta^{2}=.02$ (small effect), and both groups of parents presented with average intelligence at the time of the study.

Additionally an independent-samples $t$-test was conducted to compare faking versus nonfaking parents by the WIAT-III total score. The WIAT-III data also met all assumptions of normality, and followed a normal curve. Consistent with findings regarding the WASI-II, parents in the faking group $(M=98.90, S D=23.22)$ did not significantly differ from parents in the nonfaking group $(M=101.12, S D=17.42), t(60)=-.42 p=.67, \eta^{2}=.002$ (no effect) and both groups of parents presented with average reading comprehension skills at the time of the study. Thus, these group comparisons did not reveal a difference between faking-good and non-faking 
parents in IQ or reading comprehension skills.

\section{Research question 3. Does parental self-reported psychopathy impact socially} desirable responding on risk assessment measures?

Faking and non-faking parents were compared by Primary Psychopathy, Secondary Psychopathy, and Total scores on the LSRP. These separate variables were compared, to assess for potential differences between faking and non-faking parents on Factor 1 psychopathy characteristics (e.g., lying, manipulation) and Factor 2 psychopathy characteristics (e.g., behavioral impulsivity, antisocial behaviors), as well as the overall intensity, or severity of psychopathic personality traits. To best test this question, a multivariate analysis of variance was utilized, with faking and non-faking parents as the comparison, or fixed variable, and each subscale on the LSRP as dependent variables. Each of the scores (e.g., Primary Psychopathy, Secondary Psychopathy, and Total Score) on the LSRP violated assumptions of normality, and were positively skewed and kurtotic. Therefore, they were log-transformed, and subsequently met assumptions for normality. However, there was not an effect of faking on any of the scores of the LSRP, $F(2,59)=2.30, p=.10, \eta^{2}$ partial $=.09$ (small effect). A comparison of the means of each scale indicated that faking and non-faking parents had equal scores on the Primary Psychopathy subscale of the LSRP $(M=24.50, S D=6.08$ and $M=24.55, S D=6.52$, respectively). Parents in the faking group had slightly lower scores on the Secondary Psychopathy subscale $(M=19.31, S D=5.70)$ than non-faking parents $(M=22.50, S D=7.86)$. Additionally, parents in the faking group also had slightly lower total scores on the LSRP ( $M=$ 43.81, $S D=10.82)$ than non-faking parents $(M=47.05, S D=13.03)$.

Research question 4. Does parental positivity bias impact socially desirable responding on risk assessment measures? 
Although there was not an overall learning bias, attitude generalization, or weighting bias in favor of positive or negative stimuli, it should be noted that faking and non-faking parents differed on the learning bias variable, $t(57)=2.95, p=.005$, yielding a large effect size $\left(\eta^{2}=\right.$ .13). Specifically, individuals in the faking group demonstrated a positive learning bias $(M=.14$, $S D=.19)$. Individuals in the non-faking group did not demonstrate a strong bias for either positive or negative information $(M=-.007, S D=.17)$. Additionally, faking and non-faking parents differed on the attitude generalization variable, $t(57)=2.47, p=.01$, yielding a moderate effect size $\left(\eta^{2}=.09\right)$. Again, individuals in the faking group demonstrated positive attitude generalization to novel stimuli $(M=.15, S D=.30)$, and individuals in the non-faking group did not demonstrate an attitude generalization strongly favoring positive or negative information $(M$ $=-.03, S D=.27)$ in the test phase on BeanFest. Similar to attitude generalization, when testing for a weighting bias, faking and non-faking parents had significantly different scores, $t(57)=$ $2.67, p=.01$, yielding a moderate effect size $\left(\eta^{2}=.11\right)$. Individuals in the faking group demonstrated a weighting bias favoring positive stimuli $(M=.14, S D=.34)$, while non-faking parents did not demonstrate a clear bias $(M=-.08, S D=.28)$. However, there was considerable variability around the mean across the attitude generalization and weighting bias variables. Table 5 shows comparisons between faking and non-faking parents across study outcomes.

\section{Exploratory Variables}

\section{Exploratory question 1. Which additional variables are significantly related to}

\section{socially desirable responding on the CAPI?}

As mentioned previously, the CAPI Lie Scale was used in the exploratory analyses, due to the underpowered sample size and unequal groups of faking and non-faking parents. All primary outcome variables (e.g., treatment group, WASI-II, WIAT-III, LSRP, BeanFest learning 
bias, attitude generalization, and weighting bias) as well as additional exploratory variables (e.g., MCSDS and BDI-II scores, whether or not the parent had experienced trauma, and whether or not the parent had a child with either learning or behavior problems) were entered with the Lie Scale into a correlational matrix. The CAPI Lie Scale was significantly negatively correlated with WASI-II, $r=-.42, p=.001$ and WIAT-III scores, $r=-.35, p=.007$. The Lie Scale was positively correlated with the MCSDS score. $r=.73, p=.000$, and the BeanFest learning bias $r=$ $.28, p=.02$, and weighting bias, $r=.26, p=.04$ variables. Table 6 presents the full correlational matrix.

\section{Exploratory question 2. Which variables most strongly predict socially desirable}

\section{responding on the CAPI?}

A stepwise multiple regression using the forward method was conducted, using predictors that were found to be significantly associated with the CAPI Lie Scale in previous analyses. These variables included the WASI-II and WIAT-III scores, the MCSDS total score, and the BeanFest learning bias and weighting bias variables. Before interpreting results from this model, diagnostic tests were run, and the model met all assumptions for a multiple regression, including absence of high multicollinearity (variance inflation factor $=1.0$ ), homoscedasticity of data, and independence of errors (Durbin-Watson test $=1.42$ ). In the prediction model, the MCSDS score was found to significantly predict the Lie Scale on the CAPI, $F(1,51)=60.29, p=.000$, and the overall model accounted for $54 \%$ of the variance of socially desirable responding on the Lie Scale $\left(R^{2}=.54\right.$, Adjusted $\left.R^{2}=.53\right)$. Specifically, higher scores on the MCSDS were predictive of higher scores on the Lie Scale. Parent intelligence, measured by the WASI-II, approached significance, but was not included in the final prediction model. Table 7 shows the raw and standardized regression coefficients for the MCSDS score in the final prediction model. Raw 
betas are also presented for the excluded variables in the model.

A stepwise multiple regression using the forward method was also conducted predicting the CAPI Lie Scale from the primary outcome variables: treatment setting, WASI-II and WIATIII scores, the LSRP Primary Psychopathy score, and the BeanFest learning bias variable. Before interpreting results from this model, diagnostic tests were run, and the model met all assumptions for a multiple regression, including absence of high multicollinearity (variance inflation factor $=$ 1.0), homoscedasticity of data, and independence of errors (Durbin-Watson test $=2.39$ ). In this prediction model, the WASI-II score was found to significantly predict the Lie Scale on the CAPI, $F(1,53)=9.68, p=.003$, and the overall model accounted for $15 \%$ of the variance of socially desirable responding on the Lie Scale $\left(R^{2}=.15\right.$, Adjusted $\left.R^{2}=.13\right)$. Specifically, lower scores on the WASI-II were predictive of higher scores on the Lie Scale, or, a higher level of socially desirable responding on the CAPI. The LSRP Primary Psychopathy score and the BeanFest learning bias variables approached significance, but were not included in the final prediction model. Table 8 shows the raw and standardized regression coefficients for the WASIII score in the final prediction model. Raw betas are also presented for the excluded variables in the model.

\section{Discussion}

The current investigation added to the literature regarding parental characteristics associated with faking-good responding on the Child Abuse Potential Inventory (Milner, 1986), by conducting a prospective study, incorporating a multimethod approach to data collection (e.g., self-report, performance-based measures, and behavioral tasks), and recruiting a truly "real world" and at-risk sample. A between-subjects design was used to test for potential differences between parents with an invalid profile on the CAPI due to faking-good behavior and parents 
without an invalid profile. In the current study, 22 (35.5\%) parents faked good on the CAPI. This percentage falls within the scope of profiles found in other studies researching the Faking-good Index; specifically, authors reported a range of $19-58 \%$ of participants with an elevated Fakinggood Index on the CAPI (Bradshaw et al., 2011; Budd et al., 2000; Carr et al., 2005; Costello \& McNeil, 2014). In the present study, faking and non-faking parents were compared across variables falling within four major domains: treatment setting, cognitive functioning (e.g., intellectual and reading comprehension ability), self-reported characteristics of psychopathy, and attitudinal variables, measured by a non-face valid behavioral task. Additionally, other exploratory variables (e.g., social desirability and depression) were considered, and parents in the study were compared across these variables as well. Results from the primary and exploratory research questions, clinical considerations, limitations of the study, and future directions for this area of research are outlined below.

\section{Primary Hypotheses}

Treatment setting. Faking-good parents were no more likely to be involved in child protective services than parents in the non-faking group, and thus the first hypothesis in this study was not supported. Interestingly, out of the 22 faking parents, over half (54.5\%) actually came from the treatment seeking without child protection group, which is much greater than the 7-11\% of "general population" parents (i.e., parents with no treatment involvement at all) noted by Milner (1986). Researchers have identified that parents who are involved in PCAs may be motivated to engage in socially desirable responding on risk assessment measures like the CAPI, due to the serious consequences (e.g., legal ramifications, modification or termination of parental rights) of these evaluations (Budd, 2001; Budd \& Holdsworth, 1996). Although a commonly held belief, this assumption that parents involved in assessments through child protective 
services have a motivation to fake good may lead to an impression of parents who do respond in a socially desirable manner as "liars" or "manipulators," which carry a distinct, negative connotation. However, as noted above, child protection parents in this study were less likely to fake good on the CAPI. The low level of faking-good on the CAPI occurred despite that, at the time of the study, these parents were involved with agencies that were monitoring their parenting practices (e.g., child advocacy center, in-home services agency partnered with the West Virginia Department of Health and Human Resources), and in some cases, parents were only getting supervised visitation with their children. Although the child protection parents were assumed to have more motivation to lie, they actually did so less than the treatment-seeking without child protection group.

Thus, the unique experience of being involved with child protective services, including undergoing the stress of being scrutinized and assessed by caseworkers, therapists, and members of a legal team, did not differentiate faking versus non-faking parents on the CAPI. The present study is the first to directly compare parents from a child protection and treatment seeking without child protect group on faking-good responding on the CAPI, and so comparison data are not available to interpret the prevalence rates of faking good in this particular sample. More research is needed, then, to see if these findings hold up in other studies.

Cognitive functioning. Results from the present study support that lower parental intelligence and reading comprehension are associated with higher socially desirable responding on the CAPI. Indeed, scores on both the WASI-II and WIAT-III demonstrated a moderate correlation with the CAPI Lie Scale ( $r=-.42$ and -.35 , respectively), and in follow-up regression analyses indicated the WASI-II scores significantly predicted the CAPI Lie scale over and above all other primary outcome variables. Although group comparisons between faking and non- 
faking parents did not yield a significant difference, on average, parents in the faking group had an IQ score that was six points lower $(M=93.45, S D=16.10)$ than parents in the non-faking group $(M=99.62, S D=18.92)$. Faking parents also had a reading comprehension score that was three points lower $(M=98.90, S D=23.79)$ than non-faking parents $(M=101.29, S D=18.13)$. A possible explanation for the nonsignficant difference was that the group comparison results may have been diminished by the small sample size of the study. Additionally, both faking and nonfaking parents already scored in the low-average to average intelligence and reading comprehension skills, so there may have been too restricted of a range in scores to detect differences. Despite the noted limitations, these results support past research from Costello and McNeil (2014) which found that faking-good parents had a lower IQ score than non-faking parents, as well as Budd et al. (2000) which found that faking-good mothers had lower reading comprehension scores than mothers with valid profiles on the CAPI.

What is interesting about the above findings is that the Faking-good Index was originally developed to identify individuals who purposefully engage in socially desirable responding outside of chance (i.e., do not demonstrate a random response pattern on the measure) (Milner, 1986). Thus, parents who fake good on the CAPI are assumed to have the intellectual savvy to engage in purposeful deception. This profile of a faking-good parent appears more consistent with that of an individual demonstrating psychopathy characteristics, like manipulation and superficial charm, and may result in negative impressions of the individual during PCAs, including discarding or discounting data the parent provides on the CAPI abuse risk scale.

In the present study, because intelligence and reading comprehension skills better differentiated faking and non-faking groups than motivation associated with child welfare involvement, reconsidering the impressions of parents who fake good on the CAPI may be 
warranted. Rather than assuming parents are high on psychopathic personality traits or simply motivated to fake good as a function of being involved in child protective services, it is suggested that more emphasis is placed on their intellectual functioning and related adaptive skills. This is an important distinction given that parents with low intellectual functioning are typically overrepresented in child welfare, and are already at a greater risk of experiencing stigma from mental health professionals, compared to parents with intelligence within normal limits (Azar et al., 2012).

As noted by Azar et al. (1998), successful parenting requires individuals to utilize a high level of cognitive resources, by attempting to set short- and long-term goals for their child, make judgments about their child's behavior, and form decisions about how best to interact and intervene with their child to meet his/her needs. Parents with intellectual deficits are more likely to have deficits in executive functioning, such as problem-solving skills and flexibility in thinking (Azar et al., 2012), which could be associated with difficulty interpreting the nuances of the forced-choice items comprising the CAPI. These individuals who are already at-risk for involvement in child protective services may be further penalized by the negative perception of a "faking-good" parent on the CAPI, if their response style is attributed to lying and manipulation. Thus, a change in perspective of a faking-good parent may help to reduce stigma associated with this responding and allow for more appropriate impressions of the parent's skills and deficits to better inform treatment recommendations.

Self-reported psychopathy. Additionally, none of the domain scores on the LSRP (i.e., Primary Psychopathy, Secondary Psychopathy, and the Total score) were significantly associated with the CAPI Lie Scale. Of note, faking $(M=24.50, S D=6.08)$ and non-faking $(M=24.55, S D$ =6.52) parents actually had almost identical Primary Psychopathy scores, which indicates that 
parents with faking-good profiles on the CAPI were no more likely to demonstrate psychopathy characteristics than non-faking parents. This is especially important because the Primary Psychopathy scale measures traits associated with deceit, charm, and manipulation, which are often assumed to be used by parents to "pull the wool over the eyes" of mental health professionals during PCAs. When interpreting these results, one could argue that parents in this study were simply lying on a face valid self-report measure, and thus, it is unclear as to whether or not the scores obtained are true indicators of the participants' psychopathy characteristics. Indeed, individuals who have high levels of psychopathic traits are typically more likely to engage in positive or negative impression management and thus, it is natural to question the ability of a self-report measure in obtaining a true view of these characteristics (Ray, Hall, Rivera-Hudson, Poythress, Lilienfeld, \& Morano, 2013).

To address this issue, Ray et al. (2013) conducted a meta-analysis investigating the association between scores on two self-report measures of psychopathy (e.g., the Psychopathic Personality Inventory and Psychopathic Personality Inventory - Revised; Lilienfeld \& Andrews, 1996; Lilienfeld \& Widows, 2005, and the LSRP; Levenson et al., 1995) and scores on five measures of socially desirable responding (including the MCSDS; Crowne \& Marlowe, 1960, Balanced Inventory of Responding; Paulhus, 1984, and the Positive Impression Management scale from the Personality Assessment Inventory; Morey, 1991). Results from the meta-analysis indicated that there was no association between the Factor 1 score on the PPI and PPI-R or the Primary Psychopathy score on the LSRP and any measure of socially desirable responding. Thus, both the PPI/PPI-R and the LSRP demonstrated that valid data could be obtained from self-report of psychopathy characteristics that were not necessarily confounded by participant socially desirable responding. 
Therefore, it is argued that faking-good parents in the present study were not endorsing personality characteristics consistent with a deceptive, manipulative individual, who shows no remorse for insincere behavior. As described previously, there were also no differences between child protection and treatment seeking without child protection parents in regard to faking-good responding on the CAPI, which is inconsistent with the typical impression of these individuals. However, results from the present study do not support this impression, and rather than being high on psychopathy, or involved in child welfare, faking-good parents were more likely to have lower intellectual functioning and reading comprehension skills, as well as a positivity bias, when compared to non-faking parents. Again, it is suggested that mental health professionals carefully consider alternative explanations regarding the characteristics of a faking-good parent.

Positivity bias. In addition to intelligence and reading comprehension skills, parental attitudinal bias, as measured by the learning bias, weighting bias, and attitude generalization scores on the BeanFest significantly differentiated faking and non-faking parents. Specifically, parents in the faking-good group demonstrated a positivity bias (i.e., the tendency for individuals to attend to, and learn positive information better than negative information), while parents in the non-faking group did not demonstrate a bias strongly favoring either positive or negative stimuli. What is also interesting is that parents in the faking-good group weighted positive information more heavily than non-faking parents when forming attitudes about novel and ambiguous stimuli. Thus, parents in the faking-good group showed a tendency to favor positive information across two distinct domains. These results are strong given that the BeanFest is a non-face valid task, and parents were not informed of the true purpose of the experiment in either the instructions or by the administrators. This study is also unique in that it demonstrated that BeanFest can be utilized with a non-college student population, by administering the task to a 
sample of individuals ranging in age, IQ, and life stressors.

The clear discrepancy between faking and non-faking groups regarding learning bias and attitude generalization indicates that perhaps, parents in the faking-good group were more likely to demonstrate attitudinal (and perhaps attirbutional) biases that are global, positive, and at times unrealistic (i.e., selectively attending to positive information in the environment even when it is incorrect to do so). The presence of a positivity bias could affect how parents respond on the CAPI in many ways. For one, because the CAPI utilizes forced-choice responding, when given the opportunity to only pick one of two answers (much like parents being asked to either classify a bean on BeanFest as either positive or negative) parents with a positivity bias may be more likely to default in choosing the more positive interpretation of the answer over the more negative interpretation, even if it is not necessarily the "best" answer. This is especially problematic because the CAPI contains "all or none" questions, like "I am always a good person," or "I am always happy with what I have." Paired with low intelligence, these parents may not have the executive functioning skills necessary to carefully reflect on whether or not each item on the CAPI truly reflects their traits, attitudes, or behavior (e.g., whether or not they truly are good all of the time, or are always happy with what they have).

Parents with a positivity bias may also present with a skewed interpretation of the actual purpose of the CAPI during PCAs. Although instructions on the CAPI indicate that parents should answer honestly on each item, these individuals may view completing the CAPI as an opportunity (along with completing other measures during PCAs) to highlight their strengths, and may not understand or consider the implications associated with responding in an overly favorable manner. Thus, parents who demonstrate a positivity bias may be penalized, without their awareness, by the negative impression associated with a faking-good profile on the CAPI. 
Another important consideration is that faking-good parents may actually be demonstrating a self-serving attributional bias. One interesting conceptualization of an attributional bias comes from work developed by Paulhus (1998; 2002), which posits that individuals who respond in a socially desirable manner may either demonstrate traits related to self-deception or impression management. Notably, these two traits have been found to be weakly associated with each other and are thought to represent two distinct constructs (Paulhus, 2002). Self-deception was defined by Paulhus as including both self-enhancement (i.e., the tendency to hold overly optimistic or positive views of oneself, even when incongruent with one's behavior or ratings from others), and self-denial (i.e., selectively ignoring one's negative traits or behaviors) (Paulhus, 2002). This differs from socially desirable responding related to impression management, which is defined as a calculated attempt at building up an impression of oneself based on what others believe to be favorable (Paulhus \& Reid, 1991).

Self-deception, and in particular, self-enhancement have been shown to be psychologically adaptive, and self-enhancers have been found to report higher levels of selfesteem and emotional well-being, compared to non-enhancers (Paulhus, 1998; Taylor \& Armor, 1996). Interestingly, although self-enhancers tend to make positive first impressions, these impressions become less favorable over time (Colvin, Block, \& Funder, 1995; Paulhus, 1998). Research has shown that during an initial meeting between self-enhancers and trained observers (who had no prior relationship to the subjects) self-enhancers were rated as intelligent, interesting, and displaying a high level of confidence. However, at week seven, the observers rated these individuals as arrogant, bossy, and overstating their abilities (Paulhus, 1998).

When applied to faking-good parents during PCAs, the concept of self-deception and particularly self-enhancement may have utility in determining why parents engage in this 
response pattern. Perhaps parents who fake good on the CAPI are higher in self-enhancement than parents who do not, and thus, truly believe in the unrealistically positive answers they submit. However, as described above, mental health professionals may believe that selfenhancing parents are purposefully exaggerating their strengths, or bragging about their positive qualities and characteristics, and in essence, may view self-enhancers are engaging in positive impression management, although these two constructs have been shown to be weakly related.

This study was the first to directly assess parental attitudes in the context of faking-good responding on the CAPI. As stated above, the BeanFest was successfully administered to a "real world" sample, with varying levels of IQ, reading comprehension, education, and stressors. It produced a clear difference between faking and non-faking parents in their tendency to weight positive over negative information in their environment. In light of these findings, replication is needed to assess whether or not similar patterns emerge between faking and non-faking parents in future studies. It may also be indicated to study the use of BeanFest with parents who are undergoing PCAs, to see if an attitudinal bias (i.e., how much weight faking-good parents place on either positive or negative information) holds with parents outside of an analog setting.

It may also be indicated to assess whether or not faking-good parents demonstrate high self-enhancement or impression management traits, and whether or not these scores are associated with the learning bias and attitude generalization variables on the BeanFest. The Balanced Inventory of Desirable Responding (BIDR; Paulhus, 1988) was developed to measure both of these traits in respondents. It is a 40-item self-report measure using a 7-point Likert scale, and has been widely used in research with undergraduate samples and within the workplace. Although a review of the literature does not indicate that the BIDR has been used specifically with parents, and parents within child welfare, it is still suggested that the BIDR could be a 
useful measure to help differentiate parents who are truly demonstrating a positivity bias, versus parents who are deliberately engaged in positive impression management. Therefore, it would be interesting to see if scores on the BIDR, particularly related to the self-enhancement items, are associated with scores on the BeanFest, and the CAPI Lie Scale. If self-enhancement items, but not impression management items are strongly associated with these behavioral and self-report measures, then it may be more strongly argued that parents who engage in faking-good behavior are higher on self-enhancement than parents with valid profiles.

\section{Exploratory Variables: Social Desirability and Depression}

The total score on the Marlowe Crowne Social Desirability Scale (Crowne \& Marlowe, 1960) was strongly associated with the Lie Scale on the CAPI, $r=.73$, where higher scores on the MCSDS were associated with higher levels of faking good on the CAPI. Additionally, the MCSDS score accounted for over half of the variance (54\%) of the CAPI Lie Scale when entered into a stepwise regression, demonstrating a sound link between the two measures. Although early research (e.g., Robertson \& Milner, 1983; 1985b) demonstrated that the CAPI and MCSDS were moderately associated with each other $(r=.32)$, and were both able to detect a high percentage of faking-good participants (CAPI Lie Scale: 78.8\% and MCSDS: 88\%), little research appears to have been recently published which compare the two scales. The current study, then, expands on this early research base by providing updated information on the strength of the association between both scales.

Consistent with the CAPI Lie Scale, the MCSDS was also negatively correlated with scores on the WASI-II $(r=.29)$, indicating that lower parental intelligence yielded higher social desirability. These scores were not significantly correlated with any of the scores on the LSRP. Again, the modest association between the MCSDS and WASI-II, as well as the lack of 
association between the MCSDS and LSRP scores indicates parents who respond in a socially desirable manner may not necessarily be more manipulative than parents who do not. These parents may simply be responding in a different, not necessarily problematic or deceptive way on self-report measures during PCAs.

By contrast, there was not a significant association between the CAPI Lie Scale and scores on the BDI-II. Interestingly, faking and non-faking parents had almost identical scores on this measure, and both were classified as being in the "mild" range (e.g., average score was approximately 10; scores from 10-18 indicate mild symptoms of depression; Beck et al., 1996) and this restricted range may have diminished any differences between the two groups. These results are inconsistent with data from the Costello and McNeil (2014) study, which found that faking-good parents had lower BDI (Beck et al., 1961) scores than non-faking parents; although parents in this study scored within the minimal to mild range (e.g., $M=6.97$ for faking parents and $M=13.91$ for non-faking parents; Costello \& McNeil, 2014).

Despite the fact that the BDI-II did not discriminate between faking and non-faking parents it is still recommended for use in PCAs due to its prevalence in screening for depressive disorders in both research and clinical work. The BDI-II can yield important information regarding parents' attitudinal style, and it would be interesting to see if future research is able to detect an association between the BeanFest learning bias, weighting bias, or attitude generalization variables and scores on the BDI-II with a child welfare sample. Results from this study also disconfirm that faking parents were simply "lying" on the BDI-II, given the almost identical scores between the two groups. Thus, despite is high face validity, the BDI-II was shown to be a useful measure for assessing parental depressive symptoms in the context of PCAs. 


\section{Clinical Considerations}

As emphasized throughout this paper, mental health professionals should consider using an alternate interpretation of parents who engage in faking-good responding on the CAPI. Data obtained from this investigation demonstrated that faking parents are not necessarily higher on psychopathy traits than non-faking parents, nor are they any more likely to display motivation to fake good because of their involvement in the child welfare system. However, parents' fakinggood CAPI profiles are often thought of as savvy manipulators, attempting to purposefully deceive mental health professionals. The impression that an individual is purposefully lying or manipulating typically carries a weighty negative connotation, and this impression may skew the assessment of the parent during PCAs, including discarding data from the CAPI or discounting other information obtained from the parent. Diagnostic impressions and treatment recommendations may also be altered due to a negative perception of the parent. This current study, as well as past research (e.g., Budd et al., 2000; Costello \& McNeil, 2014) support the notion that parents who engage in faking-good responding on the CAPI may not be high on psychopathy, but rather present with low intelligence and reading comprehension skills, as well as the tendency to demonstrate a positivity bias. It is argued that this conceptualization of a parent could potentially lead to (a) different strategies of assessment, aimed at setting parents up for success, and (b) different diagnostic impressions and treatment recommendations more appropriate for the parent's actual strengths and deficits.

It is also suggested that evaluators conducting PCAs develop a protocol about how to best use or interpret invalid CAPIs due to faking-good responding. Instead of discarding all data from an invalid profile, it may be clinically useful to examine how the CAPI Lie scale is associated with other measures included in the PCA. Implementing a "best practice" PCA should already 
consist of a multimethod approach to data collection, by examining a range of constructs including parental intelligence, knowledge and skills, social support, and psychopathology, (Budd et al., 2011). Results from the present study confirm that an IQ and reading comprehension measure should be included during PCAs to not determine the cognitive ability of the referred parent, but may also be helpful as collateral data in explaining why the parent has an elevated Faking-good Index. Parental performance on measures of intelligence and reading comprehension could offer a rich set of data related to the individual's ability to engage in critical thinking skills, which may be crucial in being able to detect the nuances of the items on the CAPI.

Additionally, the MCSDS may be a useful measure to include in PCAs, given its strong association with the CAPI Lie Scale. If similar associations emerge between scores on the MCSDS and IQ and reading comprehension scores (like in the current study), this information may confirm that parents are not necessarily purposefully lying, but may be presenting with intellectual deficits. BeanFest also differentiated between faking and non-faking parents, and may have utility as an indicator of parental learning bias and attitude formation for parents in the child welfare system. Because no other studies have used BeanFest with a child welfare population, additional research is warranted to assess whether or not the BeanFest results maintain. If these results do maintain, it may be indicated for mental health professionals to include assessments examining global attitudes and worldviews of the parent.

One such assessment is the BIDR (Paulhus, 1998), which has the ability to measure both self-enhancement and impression management traits. As stated previously, the BIDR does not appear to have been used in research with parents (and specifically with parents in the child welfare system) and so more research is indicated to assess whether or not it could be a useful 
measure in PCAs. Overall, these data, along with intelligence, reading comprehension, and MCSDS scores, may not only provide a rich source of information about the referred parent, but also help to make better sense of faking-good profiles on the CAPI.

As mentioned earlier in this paper, Milner (1986) adapted the clinical cutoff of the CAPI Lie Scale to better account for potential deficits in parental level of education. Parents with a twelfth-grade education, or less must have a score of eight or above on the Lie Scale, while parents with greater than a twelfth-grade education must have a score of seven or above. With this concept in mind, it is proposed that a similar practice should be considered based on parental IQ and socially desirable responding. If more data support the pattern that faking-good parents are low on IQ, and high on MCSDS, it may be warranted for CAPI researchers to provide a set of norms from which mental health professionals can interpret invalid profiles. Just as the Lie Scale is interpreted differently based on the parent's level of education, the broadband abuse risk score, or six corresponding domain scores may need to be interpreted differently based on parental intelligence and socially desirable responding.

The current clinical cutoff for the abuse risk score is either 166 or the more conservative 215 (Milner, 1986). Like the Lie Scale, these cutoffs may need to be altered based on a parent's intelligence and socially desirable responding scores. New cutoff points could help to retain more CAPI profiles in PCAs, instead of simply discarding them. They would also provide the same clinically useful information as the current cutoffs do for valid profiles. Norms may also help mental health professionals systematically know how to interpret data from invalid profiles, as well as deciding the best approach to take regarding using these data when developing impressions and recommendations. The need for more research including the variables described (e.g., intelligence and social desirability measures) is imperative to be able to begin determining 
the consistent patterns of responding needed to create such norms and adjust cutoff points.

Finally, it may be useful to consider renaming the CAPI Lie Scale (and by association, the Faking-good Index) if a consistent relation emerges between cognitive functioning, positivity bias, and an elevated Lie Scale. By definition, the word "lie" means "to make an untrue statement with the intent to deceive" or "to create a false or misleading impression" (MerriamWebster, 2014). This definition implies that an individual must have insight into the potential consequences that may arise from this behavior, and must purposefully create a view of $\mathrm{him} /$ herself that is qualitatively different than what he/she believes. However, a parent with low intellectual functioning may not necessarily possess the intellectual savvy to have "intent to deceive" (Merriam-Webster, 2014). Additionally, an individual harboring a self-serving attributional bias may truly hold optimistic (albeit potentially unrealistic) views of him/herself, others, and the world. This appears to be much different than an individual creating a "false or misleading impression" (Merriam-Webster, 2014). Thus, the Lie Scale on the CAPI may be identifying individuals who are not best described by the word "lie."

If more conclusive results and behavioral patterns can be obtained regarding faking-good responding on the CAPI, it may be warranted to re-think how this scale is conceptualized. As mentioned throughout this document, the name "faking good" implies a parent who is deliberately attempting to engage in socially desirable responding, outside of chance. However, parents with elevated profiles on the Faking-good Index may actually be less likely to deliberately fake good on the CAPI and more likely to either possess an overly positive, potentially naïve view of the world, or truly have intellectual deficits which prohibit them from answering in a valid way. Therefore, the conceptualization of "faking" may need to be reconsidered, as additional research is published in this area. One suggestion is considering a 
"naïveté" scale. The word "naïve" is defined as "marked by unaffected simplicity," or "deficient in worldly wisdom or informed judgment" (Merriam-Webster, 2014) and may better characterize individuals with low intellectual functioning who also have a positivity bias. When presented to a mental health professional, it is assumed that the name "lie" conjures up a very different picture than the word "naïve," and it is expected that there would be a less negative connotation associated with a "naïveté" scale than a "lie" scale.

\section{Limitations and Future Directions}

This study has multiple limitations, which should be noted. To begin, the sample size was underpowered, and an initial power analysis detected a sample size around 60 participants would only be able detect a moderate effect size at $75 \%$ power. The fact that the sample was underpowered may have resulted in a lack of differences between faking and non-faking parents, as well as within the exploratory analyses. Future studies would benefit from including more participants. In a similar area, the current study did not recruit a normal control group. The exclusion of normal controls was initially justified by completely focusing on parents receiving some level of intervention, because this sample is consistent with published research in this area (which has used samples of parents who are treatment-involved). However, given that there have been few studies which have collected data on normal or "general population" parents using the CAPI Faking-good Index, this study would have been strengthened by including a normal control group by being able to (a) provide additional information regarding the percentage of "normal" parents who fake good on the CAPI, and (b) use the normal controls as an additional comparison group.

Additionally, although the current study attempted to recruit at-risk parents, who were involved in child protective services, as well as used measures that are commonly found in PCAs 
(Budd et al., 2011), data from this study were not collected from actual PCAs, and thus, participants were assessed under an analog condition. More powerful, and potentially interesting, results may be obtained from parents who are actually experiencing a PCA, given the real-world nature of the assessment setting. Carr et al. (2005) appears to be the only study to have collected information from parents undergoing a PCA, but only included one measure (IQ) of parental characteristics. It may be warranted to compare the measures used and data collected in this study with measures used and data collected in PCAs, to see if results are similar. Given that the BeanFest has not been used, either clinically, or in research studies, until the current study, it would be useful to see if similar results are obtained with the BeanFest for parents who are actually completing PCAs. If results are similar, it may indicate a measure of parental attitude formation and generalization may be a useful component of PCAs.

Parents in this study were not asked to complete a behavioral observation task with their child, and much of the data collected was from self-report measures. Future studies would be strengthened by expanding on the multimethod data collection used in the current study, by including a behavioral observation task. Behavioral observation is often used in PCAs (Budd et al., 2011), and is a less face valid means of assessing parental functioning. It would be interesting to see if data collected from parent-child interactions during behavioral observation are associated with responding on the CAPI; one area of interest would be to discover if there are there specific behavioral patterns (either negative or positive) that are associated faking-good responding. Currently, only two of the studies described in this literature review used behavioral observation data in analyses (Budd et al., 2000; Costello \& McNeil, 2014), and did not find that behavioral observation differentiated faking and non-faking groups. Thus, more research is needed to assess to see if there is a relation between faking-good responding on the CAPI and 
behavioral observation data. Better knowledge of what parents who fake good on the CAPI actually look like during interactions with their child may provide useful information about areas for further assessment or skill building development.

Given the limitations of this study, it is proposed that there are many future areas of research. First, although this study has reported that, anecdotally, parents who fake good on the CAPI during PCAs may be thought of as "manipulative" or "liars" by mental health professionals, there are no studies that directly test this assumption. Carr et al. (2005) reported this gap in the literature, but no follow-up studies testing practitioner attitudes have been published. This differs from the child custody literature (i.e., parents undergoing child custody evaluations when going through a divorce), which has published studies assessing how faking profiles on self-report measures affect impressions of and treatment recommendations for the parent. Interestingly, Carr et al. (2005) noted that in some instances, parents who have been granted custody of their children have presented with higher K scales on the MMPI-2 (indicating higher defensiveness) than parents who have not been granted custody (Otto \& Collins, 1995). Thus, in the case of child custody disputes, parents may actually benefit from response distortion on self-report measures. In light of these findings, conducting qualitative research, by gathering opinions of mental health professionals who conduct PCAs would fill a current gap in the literature. Specifically, it would be interesting to get more data on what professionals actually think about invalid profiles (and parents with these profiles) on the CAPI. Additionally, it would also be interesting to understand how faking profiles impact diagnostic impressions and treatment recommendations during PCAs.

Second, there are no published prospective studies that have examined the long-term impact of faking good on the CAPI. Specifically, longitudinal research is needed to compare 
faking and non-faking parents across treatment participation (including attendance and adherence to treatment), outcomes at post-treatment, and perhaps most importantly, abuse recidivism rates. Costello and McNeil (2014) compared faking versus non-faking parents on physical abuse recidivism and found no differences between the two groups. However, their investigation was a retrospective study, using secondary data analysis, and is the only study published that has addressed long-term outcomes of faking-good parents. Despite the identification that fakinggood responding negatively affects information collected during PCAs, there are no data which examine whether or not this actually has an impact on parent and child outcomes. It is difficult to make assumptions about the magnitude mental health professionals should be placing on whether or not a parent fakes good on the CAPI if there are no data to show what how this response style actually affects parenting, especially change in parenting over time. Thus, perhaps the most important future direction for research in this area is to organize and implement prospective longitudinal studies assessing for the impact of faking good responding on the CAPI on parent and child treatment outcome variables.

Finally, it cannot be emphasized enough that more research is needed in the realm of what differentiates faking versus non-faking parents on the CAPI. The present study included already tested variables (e.g., IQ and reading comprehension), as well as unique methods of measurement (e.g., BeanFest) to attempt to answer this question. Future research would benefit from including similar assessment measures (as well as social desirability measures like the MCSDS and BIDR) to see if stronger patterns of parental characteristics emerge. A better understanding of what truly differentiates faking versus non-faking parents on the CAPI may lead to better interpretation and use of invalid profiles during PCAs, as well as a deeper understanding of what these parents actually need in regard to treatment recommendations. 


\section{Summary and Conclusions}

Although this study had notable limitations, it added to the dearth of literature on fakinggood responding on the Child Abuse Potential Inventory (Milner, 1986) by utilizing a multimethod, prospective study with a truly "at-risk" sample. Results from this study support that there are discrete parental characteristics which may differentiate faking versus non-faking parents. Specifically, the present investigation supported previous work which has indicated that parents who fake good on the CAPI presented with lower intellectual functioning and reading comprehension skills. Additionally, the current study is unique because faking parents demonstrated a discrete positivity bias, which differed from non-faking parents who demonstrated a discrete negativity bias. Faking parents were no more likely to be involved in the child welfare system, or endorse self-reported psychopathy symptoms, compared to non-faking parents. These findings suggest that characterizing parents with an elevated Faking-good Index on the CAPI during PCAs as "liars" or "manipulators" may not necessarily capture the true strengths and deficits of the referred parent.

Therefore, although results from this investigation added to the small literature base in this area, they also generated many unanswered questions, which need additional research studies to answer. Perhaps most importantly, longitudinal research studies should consider the long-term impact of faking-good responding on parent and child outcomes during and after treatment involvement, as well as future abuse recidivism. More data are needed regarding how mental health professionals view, interpret, and use information from invalid faking-good profiles on the CAPI. Additionally, it would be helpful to assess for patterns of parental characteristics associated with faking-good responding on the CAPI from individuals who are actively involved in PCAs. Despite these unanswered questions, the current study does support 
the idea that parents who have an elevated Faking-good Index on the CAPI may be better characterized as individuals with low cognitive functioning and a greater tendency to hold global and stable positive views of themselves, others, and the world, rather than individuals who are purposefully lying or faking on the measure.

With these data in mind, mental health clinicians conducting PCAs should be cautious of their interpretations of parents with faking-good CAPI profiles, and carefully consider all of the information they obtain from the multimethod approach to these evaluations. This careful consideration, and flexibility in forming impressions about parents who engage in faking-good responding may help to form more appropriate diagnostic impressions and treatment recommendations Perhaps most importantly, these impressions and recommendations can set both the parent, and his/her child up for the greatest success, and safety, in the future. 


\section{References}

Abidin, R. R. (1995). Parenting Stress Index: Professional Manual ( ${ }^{\text {rd }}$ ed.). Odessa, FL: Psychological Assessment Resources.

Abramson, L. Y., \& Alloy, L.B. (1980). Depression, nondepression, and cognitive illusions: Reply to Schwartz. Journal of Experimental Psychology: General, 110(3), 436-447. doi: $10.1037 / 0096-3445.110 .3 .436$

Abramson, L. Y., Alloy, L. B., Hogan, M. E., Whitehouse, W. G., Cornette, M., Akhavan, S., et al. (1998). Suicidality and cognitive vulnerability to depression among college students. A prospective study. Journal of Adolescence, 21, 473-487. doi: 10.1006/jado.1998.0167

Achenbach, T. M. (1991). Integrative guide for the 1991 CBCL/4-18, YSR, and TRF profiles. Burlington, VT: University of Vermont, Department of Psychiatry.

American Psychological Association Committee on Professional Practice and Standards (2013). Guidelines for psychological evaluations in child protection matters. Washington, DC: American Psychological Association.

Aragona, J. A. (1983). Physical child abuse: An interactional analysis. Doctoral dissertation, University of South Florida. Dissertation Abstracts International, 44, $1225 \mathrm{~B}$.

Azar, S. T., Lauretti, A. F., \& Loding, B. V. (1998). The evaluation of parental fitness in termination of parental rights cases: A functional-contextual perspective. Clinical Child and Family Psychology Review, 1(2), 77-100. doi: 10.1023/A:1021883611965

Azar, S. T., Robinson, D. R., Hekimian, E., Twentyman, C. T. (1984). Unrealistic expectations and problem-solving ability in maltreating and comparison mothers. Journal of Consulting and Clinical Psychology, 52, 687-691. doi: 10.1037/0022-006X.52.4.687

Azar, S. T., Robinson, L. R., \& Proctor, S. N. (2012). Chronic neglect and services without 
borders: A guiding model for social service enhancement to address the needs of parents with intellectual disabilities. Journal of Mental Health Research in Intellectual Disabilities, 5, 130-156. doi: 10.1080/19315864.2011.592238

Azar, S. T., \& Rohrbeck, C. A. (1986). Child abuse and unrealistic expectations: Further validation of the Parent Opinion Questionnaire. Journal of Consulting and Clinical Psychology, 54, 867-868. doi: 10.1037/0022-006X.54.6.867

Azar, S. T., Stevenson, M. T., \& Johnson, D. R. (2012). Intellectual disabilities and neglectful parenting: Preliminary findings on the role of cognition in parenting risk. Journal of Mental Health Research in Intellectual Disabilities, 5(2), 94-129. doi: $10.1080 / 19315864.2011 .615460$

Barone, N. M., Weitz, E. I., \& Witt, P. H. (2005). Psychological bonding evaluations in termination of parental rights cases. The Journal of Psychiatry and Law, 33, 387-411.

Barrera, M. (1981). Social support in the adjustment of pregnant adolescents: Assessment issues. In B. H. Gottlieb (Ed.), Social networks and social support (pp. 69-96). Beverly Hills, CA: Sage.

Barron, F. (1953). An ego-strength scale which predicts response to psychotherapy. Journal of Consulting Psychology, 17, 327-333. doi: 10.1037/h0061962

Bavolek, S. J. (1984). Handbook for the Adult-Adolescent Parenting Inventory. Eau Claire, WI: Family Development Associates, Inc.

Beck, A. T., Epstein, N., Brown, G., \& Steer, R. A. (1998). An inventory for measuring clinical anxiety: Psychometric properties. Journal of Counseling and Clinical Psychology, 56, 893-897. doi: 10.1037/0022-006X.56.6.893

Beck, A. T., Steer, R. A., \& Brown, G. K. (1996). Manual for the Beck Depression Inventory-II. 
San Antonio, TX: Psychological Corporation.

Beck, A.T., Ward, C. H., Mendelson, M., Mock, J., \& Erbaugh, J. (1961). An inventory for measuring depression. Archives of General Psychiatry, 4, 561-571.

Book, A. S., Holden, R. R., Starzyk, K. B., Wasylkiw, L., \& Edwards, M. J. (2006).

Psychopathic traits and experimentally induced deception in self-report assessment. Personality and Individual Differences, 41, 601-608. doi: 10.1016/j.paid.2006.02.011

Book, A. S., Quinsey, V. L., \& Langford, D. (2007). Psychopathy and the perception of affect and vulnerability. Criminal Justice and Behavior, 34(4), 531-544. doi: $10.1177 / 0093854806293554$

Bradshaw, K. M., Donohue, B., Cross, C., Urgelles, J., \& Allen, D. N. (2011). Examination of the relationship between parental satisfaction and child maltreatment potential while considering social desirability. Journal of Family Violence, 26, 545-549. doi: $10.1007 / \mathrm{s} 10896-011-9389-\mathrm{x}$

Brinkley, C. A., Schmitt, W. A., Smith, S. S., \& Newman, J. P. (2001). Construct validation of a self-report psychopathy scale: Does Levenson’s Self-Report Psychopathy Scale measure the same constructs as Hare's Psychopathy Checklist - Revised? Personality and Individual Differences, 31, 1021-1038. doi: 10.1016/S0191-8869(00)00178-1

Browne, A., \& Finkelhor, D. (1986). Impact of child sexual abuse: A review of the research. Psychological Bulletin, 99(1), 66-77.

Budd, K. S. (2001). Assessing parenting competence in child protection cases: A clinical practice model. Clinical Child and Family Psychology Review, 4(1), 1-18. doi: 10.1023/A:1009548509598

Budd, K. S. (2005). Assessing parenting capacity in a child welfare context. Children and Youth 
Services Review, 27, 429-444. doi: 10.1016/j.childyouth.2004.11.008

Budd, K. S., Connell, M, \& Clark, J. R. (2011). Evaluation of parenting capacity in child protection. Oxford University Press: New York, NY.

Budd, K. S., Heilman, N. E., \& Kane, D. (2000). Psychosocial correlates of child abuse potential in multiply disadvantaged mothers. Child Abuse \& Neglect, 24(5), 611-625. doi: $10.1016 / \mathrm{S} 0145-2134(00) 00122-8$

Budd, K. S., \& Holdsworth, M. J. (1996). Issues in clinical assessment of minimal parenting competence. Journal of Clinical Child Psychology, 25(1), 2-14. doi: 10.1207/s15374424jccp2501_1

Butcher, J. N., Graham, J. R., Ben-Porath, Y. S., Tellegen, A., \& Dahlstrom, W. G. (2001). Minnesota Multiphasic Personality Inventory -2 (MMPI-2): Manual for administration and scoring (rev. ed.). Minneapolis, MN: University of Minnesota Press.

Butcher, J. N., Morfitt, R. C., Rouse, S. V., \& Holden, R. R. (1997). Reducing MMPI-2 defensiveness: The effect of specialized instructions on retest validity in a job applicants sample. Journal of Personality Assessment, 68, 385-401. doi:

10.1207/s15327752jpa6802_9

Carr, G. D., Moretti, M. M., \& Cue, B. J. H. (2005). Evaluating parenting capacity: Validity problems with the MMPI-2, PAI, CAPI, and ratings of child adjustment. Professional Psychology: Research and Practice, 36(2), 188-196. doi: 10.1037/0735-7028.36.2.188

Chaffin, M. (2004). Physical Abuse Treatment Outcome Project: Application of Parent-Child Interaction Therapy (PCIT) to Physically Abusive Parents (Dataset). Available from National Archive on Child Abuse and Neglect Web Site, http://www.ndacan.cornell.edu.

Chaffin, M., \& Valle, L. A. (2003). Dynamic prediction characteristics of the Child Abuse 
Potential Inventory. Child Abuse \& Neglect, 27, 463-481. doi: 10.1016/S01452134(03)00036-X

Chaffin, M., Silovsky, J. F., Funderburk, B., Valle, L. A., Brestan, E. V., Balachova, T., Jackson, S., Lensgraf, J., \& Bonner, B. L. (2004). Parent-child interaction therapy with physically abusive parents: Efficacy for reducing future abuse reports. Journal of Consulting and Clinical Psychology, 72(3), 500-510. doi: 10.1037/0022-006X.72.3.500

Choate, P. W. (2009). Parenting capacity assessments in child protection cases. The Forensic Examiner, 18(1), 52-59.

Cleckley, H. (1976). The mask of sanity ( $5^{\text {th }}$ ed.). St. Louis, MO: Mosby.

Cohen, J. (1988). Statistical power analysis for the behavioral sciences $\left(2^{\text {nd }}\right.$ ed.). New York: Academic Press.

Colvin, C. R., \& Block, J. (1994). Do positive illusions foster mental health? An examination of the Taylor and Brown formulation. Psychological Bulletin, 116(1), 3-20. doi: 10.1037/0033-2909.116.1.3

Colvin, C. R., Block, J., \& Funder, D. C. (1995). Overly positive self-evaluations and personality: Negative implications for mental health. Journal of Personality and Social Psychology, 68, 1152-1162. doi: 10.1037/0022-3514.68.6.1152.

Conklin, L. R., Strunk, D. R., \& Fazio, R. H. (2009). Attitude formation in depression: Evidence for deficits in forming positive attitudes. Journal of Behavior Therapy and Experimental Psychiatry, 40(1), 120-126. doi: 10.1016/j.jbtep.2008.07.001

Costello, A. H., \& McNeil, C. B. (2014). Differentiating faking-good parents from non-faking parents on the Child Abuse Potential Inventory. Journal of Family Violence, 29(1), 7988. doi: 10.1007/s10896-013-9557-2 
Crowne, D. P., \& Marlowe, D. (1960). A new scale of social desirability independent of psychopathology. Journal of Consulting Psychology, 24, 349-354. doi: $10.1037 / \mathrm{h} 0047358$

Currie, J., \& Widom, C. S. (2010). Long-term consequences of child abuse and neglect on adult economic well-being. Child maltreatment, 15(2), 111-120. doi: $10.1177 / 1077559509355316$

Derogatis, L. R. (1983). Symptom Checklist-90 Revised administration, scoring \& procedures manual-II. Towson, MD: Clinical Psychometric Research.

Ellis, E. M. (2001). Guidelines for conducting parental fitness evaluations. American Journal of Forensic Psychology, 19(1), 5-40.

Epstein, M. K., Poythress, N. G., \& Brandon, K. O. (2006). The self report psychopathy scale and passive avoidance: A validation of race and gender effects. Assessment, 13, 197-207. doi: $10.1177 / 1073191105284992$

Fang, X., Brown, D. S., Florence, C. S., \& Mercy, J. A. (2013). The economic burden of child maltreatment in the United States and implications for prevention. Child Abuse and Neglect, 36 (2), 156-165. doi: 10.1016/j.chiabu.2011.10.006

Fazio, R. H., Eiser, J. R., \& Shook, N. J. (2004). Attitude formation through exploration: Valence asymmetries. Journal of Personality and Social Psychology, 87(3), 293-311. doi: 10.1037/0022-3514.87.3.293

Field, A. (2009). Discovering statistics using SPSS ( ${ }^{\text {rd }}$ ed.). Thousand Oaks, CA: Sage Publications, Inc.

Field, T. (1995). Psychologically depressed patients. In M. H. Bornstein (Ed.), Handbook of parenting: Vol 4. Applied and practical parenting (pp. 85-99). Mahwah, NJ: Erlbaum. 
First, M. B., Spitzer, R. L., Miriam, G., \& Williams, J. B. W. (2002). Structured clinical interview for the DSM-IV-TR Axis I disorders, research version, non-patient edition (SCID-I/NP). New York: Biometrics Research, New York State Psychiatric Institute.

Fulton, A. M., Murphy, K. R., \& Anderson, S. L. (1991). Increasing adolescent mothers' knowledge of child development: An intervention program. Adolescence, 26, 73-81.

Gardner, R. A. (1982). Family evaluations in child custody litigation. Creative Therapeutics. Hamilton, M. (1960). A rating scale for depression. Journal of Neurological Neurosurgical Psychiatry, 23, 56-62. doi: 10.1136/jnnp.23.1.56

Hare, R. D. (1991). The Hare Psychopathy Checklist-Revised. Toronto, Ontario, Canada: MultiHealth Systems.

Hare, R. D. (2003). The Hare Psychopathy Checklist-Revised ( ${ }^{\text {nd }}$ ed.). Toronto, Ontario, Canada: Multi-Health Systems.

Hare, R. D., \& Neumann, C.S. (2008). Psychopathy as a clinical and empirical construct. Annual Review of Clinical Psychology, 4, 217-246. doi:

10.1146/annurev.clinpsy.3.022806.091452

Haskett, M. E., Scott, S. S., \& Fann, K. D. (1995). Child Abuse Potential Inventory and parenting behavior. Relationships with high-risk correlates. Child Abuse \& Neglect, 19(12), 1483-1495. doi: 10.1016/0145-2134(95)00107-4

Hathaway, S. R., \& McKinley, J. C. (1943). Manual for the Minnesota Multiphasic Personality Inventory. Minneapolis: University of Minnesota Press.

Holden, R. R. (1996). Holden Psychological Screening Inventory (HPSI). Toronto, Ontario: Multi-Health Systems.

Inquisit 3.0.6.0 (Computer software). (2012). Seattle, WA: Millisecond Software. 
Jastak, S., \& Wilkinson, G. S. (1984). Wide Range Achievement Test-Revised Administration manual. Wilmington, DE: Jastak Associates.

Jellinek, M. S., Murphy, J. M., Poitrast, F. Quinn, D., Bishop, S. J., \& Goshko, M. (1992). Serious child mistreatment in Massachusetts: The course of 206 children through the courts. Child Abuse \& Neglect, 16, 179-185. doi: 10.1016/0145-2134(92)90026-N

Kahneman, D., \& Tversky, A. (1988). Prospect theory: An analysis of decision under risk. In P. Gärdenfors, N.-E. Sahlin (Eds.), Decision, probability, and utility: Selected readings, Cambridge University Press, New York (1988), pp. 183-214.

Kalich, L., Carmichael, B., Masson, T., Blacker, D., \& Urquiza, A. (2007). Evaluating the evaluator: Advice for legal professionals in assessing the competency of termination of parental rights evaluations. Psychiatry and Law, 35, 365-397.

Kaufman, A. S., \& Kaufman, J. L. (1990). Manual for the Kaufman Brief Intelligence Test. Circle Pines MN: American Guidance Service.

Kaufman, A. S., \& Lichtenberger, E. O. (1999). Essentials of the WAIS-III assessment. New York: Wiley.

Langdon, P. E., Clare, I. C. H., \& Murphy, G. H. (2010). Measuring social desirability amongst men with intellectual disabilities: The psychometric properties of the Self- and OtherDeception Questionnaire-Intellectual Disabilities. Research in Developmental Disabilities, 31, 1601-1608. doi: 10.1016/j.ridd.2010.05.001

Leeb, R. T., Paulozzi, L., Melanson, C., Simon, T., \& Arias, I. (2007). Child Maltreatment Surveillance: Uniform Definitions for Public Health and Recommended Data Elements, Version 1.0. Atlanta (GA): Centers for Disease Control and Prevention, National Center for Injury Prevention and Control. 
Levenson, M. R., Kiehl, K. A., \& Fitzpatrick, C. M. (1995). Assessing psychopathic attributes in a noninstitutionalized population. Journal of Personality and Social Psychology, 68, 151-158. doi: 10.1037/0022-3514.68.1.151

Lewinsohn, P. M., Mischel, W., Chaplin, W., \& Barton, R. (1980). Social competence and depression: The role of illusory self-perceptions. Journal of Abnormal Psychology, 89(2), 203-212. doi: 10.1037/0021-843X.89.2.203

Lilienfeld, S. O., \& Andrews, B. P. (1996). Development and preliminary validation of a selfreport measure of psychopathic personality traits in a noncriminal population. Journal of Personality Assessment, 66, 488-524. doi: 10.1207/s15327752jpa6603_3

Lilienfeld, S. O., \& Widows, M. R. (2005). Psychopathy Personality Inventory - Revised (PPIR) professional manual. Odessa, FL: Psychological Assessment Resources.

Lynam, D. R., Whiteside, S., \& Jones, S. (1999). Self-reported psychopathy: A validation study. Journal of Personality Assessment, 73, 110-132. doi: 10.1207/S15327752JPA730108

McNeil, C. B., \& Hembree-Kigin, T. L. (2010). Parent-child interaction therapy. (2 ${ }^{\text {nd }}$ ed.). New York: Springer.

Merriam-Webster (2014). Lie. Retrieved from http://www.merriam-webster.com/dictionary/lie Merriam-Webster (2014). Naïve. Retrieved from http://www.merriam-webster.com/dictionary/naive

Mezulis, A. H., Abramson, L. Y., Hyde, J. S., \& Hankin, B. L. (2004). Is there a universal positivity bias in attributions? A meta-analytic review of individual, developmental, and cultural differences in the self-serving attributional bias. Psychological Bulletin, 130(5), 711-747. doi: 10.1037/0033-2909.130.5.711

Miller, T. R., Handal, P. J., Gilner, F. H., \& Cross, J. F. (1991). The relationship of abuse and 
witnessing violence on the Child Abuse Potential Inventory with Black adolescents. Journal of Family Violence, 6, 351-363. doi: 10.1007/BF00980538

Milner, J. S. (1982). Development of a lie scale for the Child Abuse Potential Inventory. Psychological Reports, 50, 871-874. doi: 10.2466/pr0.1982.50.3.871

Milner, J. S. (1986). CAPI Inventory Manual, 2nd Ed., An Interpretive Manual for the CAPI Inventory. Psychological Assessment Resources, Lutz, FL.

Milner, J. S. (1994). Assessing physical child abuse risk: The Child Abuse Potential Inventory. Clinical Psychology Review, 14, 547-583. doi: 10.1016/0272-7358(94)90017-5

Milner, J. S., Charlesworth, J. R., Gold, R. G., Gold, S. R., \& Friesen, M. R. (1988). Convergent validity of the Child Abuse Potential Inventory. Journal of Clinical Psychology, 44, 281285. doi: $10.1016 / 0272-7358(94) 90017-5$

Milner, J. S. \& Crouch, J. L. (1997). Impact and detection of response distortions on parenting measures used to assess risk for child physical abuse. Journal of Personality Assessment, 69(3), 633-650. doi: 10.1207/s15327752jpa6903_15

Morey, L. C. (1996). An interpretive guide to the Personality Assessment Inventory (PAI). Odessa, FL: Psychological Assessment Resources.

Morsbach, \& Prinz (2006). Understanding and improving the validity of self-report of parenting. Clinical Child and Family Psychology Review, 9(1), 1-21. doi: 10.1007/s10567-006$0001-5$

Norman, R. E., Byambaa, M., De, R., Butchart, A., Scott, J. \& Vos, T. (2012). The long-term health consequences of child physical abuse, emotional abuse, and neglect: A systematic review and meta-analysis. PLoS Med, 9(11), e1001349. doi: 10.1371/journal.pmed.1 001349. 
Norris, C. J., Larsen, J. T., Crawford, E., \& Cacioppo, J. T. (2011). Better (or worse) for some than others: Individual differences in the positivity offset and negativity bias. Journal of Research in Personality, 45, 100-111. doi: 10.1016/j.jrp.2010.12.001

Otto, R. K., \& Collins, R. P. (1995). Use of the MMPI-2/MMPI-A in child custody evaluations. In Y. S. Ben-Porath, J. R. Graham, G. C. N. Hall, R. D. Hirschman, \& M. S. Zaragoza (Eds.), Forensic applications of the MMPI-2 (pp. 222-225). Thousand Oaks, CA: Sage.

Otto, R. K., Edens, J. F., \& Barcus, E. H. (2000). The use of psychological testing in child custody evaluations. Family and Conciliation Courts Review, 38, 312-340. doi: 10.1111/j.174-1617.2000.tb00578.x

Paulhus, D. L. (1984). Two-component models of socially desirable responding. Journal of Personality and Social Psychology, 46, 598-609. doi: 10.1037/0022-3514.46.3.598

Paulhus, D. L. (1998). Interpersonal and intrapsychic adaptiveness of trait self-enhancement: A mixed blessing? Journal of Personality and Social Psychology, 74(5), 1197-1205. doi: 10.1037/0022-3514.74.5.1197

Paulhus, D. L. (2002). Socially desirable responding: The evolution of a construct. In H. I. Braun, D. N. Jackson, \& D. E. Wiley (Eds.), The role of constructs in psychological and educational measurement (pp. 49-69). Mahwah, NJ: Erlbaum.

Paulhus, D. L., \& Reid, D. B. (1991). Enhancement and denial in socially desirable responding. Journal of Personality and Social Psychology, 60, 307-317. doi: 0.1037/00223514.60.2.307

Parrott, D. J., \& Zeichner, A. (2006). Effect of psychopathy on physical aggression toward gay and heterosexual men. Journal of Interpersonal Violence, 21, 390-410. doi: $10.1177 / 0886260505283153$ 
Pietri, E. S., Fazio, R. H., \& Shook, N. J. (2012). Valence weighting as a predictor of emotional reactivity to a stressful situation. Journal of Social and Clinical Psychology, 31(7), 746777. doi: $10.1521 /$ jscp.2012.31.7.746

Pietri, E. S., Fazio, R. H., \& Shook, N. J. (2013). Weighting positive versus negative: The fundamental nature of valence asymmetry. Journal of Personality, 81(2), 196-208. doi: 10.1111/j.1467-6494.2012.00800.x

Psychological Corporation (1997). Wechsler Adult Intelligence Scale - Third Edition manual. San Antonio, TX: Harcourt Brace.

Psychological Corporation (1999). Wechsler Abbreviated Scale of Intelligence manual. San Antonio, TX: Author.

Ray, J. V., Hall, J., Rivera-Hudson, N., Poythress, N. G., Lilienfeld, S. O., \& Morano, M. (2013). The relation between self-reported psychopathic traits and distorted response styles: A meta-analytic review. Personality Disorders: Theory, Research, and Treatment, 4(1), 114. doi: $10.1037 / \mathrm{a} 0026482$.

Reidy, D. E., Zeichner, A., \& Martinez, M. A. (2008). Effects of psychopathy traits on unprovoked aggression. Aggressive Behavior, 34, 319-328. doi: 10.1002/ab.20238

Robbins, L., Helzer, J., Croughan, J., \& Ratcliff, K. (1981). National Institute of Mental Health Diagnostic Interview Schedule: Its history, characteristics, and validity. Archives of General Psychiatry, 38, 381-389. doi: 10.1001/archpsyc.1981.01780290015001

Robertson, K. R., \& Milner, J. S. (1983). Construct validity of the Child Abuse Potential Inventory. Journal of Clinical Psychology, 39, 426-429.

Robertson, K. R., \& Milner, J. S. (1985a). Convergent and discriminant validity of the Child Abuse Potential Inventory. Journal of Personality Assessment, 49, 86-88. doi: 


\subsection{7/s15327752jpa4901_16}

Robertson, K. R., \& Milner, J. S. (1985b). Detection of conscious deception using the Child Abuse Potential Inventory Lie Scale. Journal of Personality Assessment, 49(5), 541-544. doi: $10.1207 / \mathrm{s} 15327752 \mathrm{jpa4905 \_ 16}$

Rohrbaugh, J. B. (2008). A comprehensive guide to child custody evaluations. New York, NY: Springer Science \& Business Media, LLC.

Runyon, M. K., Deblinger, E., Ryan, E. E., \& Thakkar-Kolar, R. (2004). An overview of child physical abuse: Developing an integrated parent-child cognitive-behavioral treatment approach. Trauma, Violence, \& Abuse, 5(1), 65-85. doi: 10.1177/1524838003259323

Shook, N. J., Fazio, R. H., \& Eiser, J. R. (2007). Attitude generalization: Similarity, valence, and extremity. Journal of Experimental Social Psychology, 43, 641-647. doi: 10.1016/j.jesp.2006.06.005

Shook, N. J., Fazio, R. H., \& Vasey, M. W. (2007). Negativity bias in attitude learning: A possible indicator of vulnerability to emotional disorders? Journal of Behavior Therapy, and Experimental Psychiatry, 38, 144-155. doi: 10.1016/j.jesp.2006.06.005

Skeem, J. L., Polaschek, D. L. L., Patrick, C. J., \& Lilienfeld, S. O. (2011). Psychopathic personality: Bridging the gap between scientific evidence and public policy. Psychological Science in the Public Interest, 12(3), 95-162. doi: $10.1177 / 1529100611426706$

Stringer, S. A., \& LaGreca, A. M. (1985). Correlates of child abuse potential. Journal of Abnormal Clinical Psychology, 13, 217-226.

Sturmey, P. (2007). Psychopathology: Depression, anxiety and related disorders. International Review of Research in Mental Retardation, 34, 197-226. 
Taylor, S. E., \& Armor, D. A. (1996). Positive illusions and coping with adversity. Journal of Personality, 64, 873-898. doi: 10.1111/j.1467-6494.1996.tb00947.x

Taylor, S. E., \& Brown, J. D. (1988). Illusion and well-being: A social psychological perspective on mental health. Psychological Bulletin, 103(2), 193-210. doi: 10.1037/00332909.103.2.193

Urquiza, A., \& Blacker, D. (2005). Psychological evaluations and the child welfare system. In P. F. Talley (Ed.). Handbook for the treatment of abused and neglected children. (pp. 83119). New York, NY: Haworth Press.

U.S. Department of Health and Human Services, Administration on Children, Youth, and Families. Child Maltreatment 2009. (Washington, DC: U.S. Government Printing Office, 2010).

Walker, C. A., \& Davies, J. (2010). A critical review of the psychometric evidence base of the Child Abuse Potential Inventory. Journal of Family Violence, 25, 215-227. doi: $10.1007 / \mathrm{s} 10896-009-9285-9$

Wechsler, D. (2009). Wechsler Individual Achievement Test-Third Edition. San Antonio, TX: NCS Pearson.

Wechsler, D. (2011). Wechsler Abbreviated Scale of Intelligence, Second Edition (WASI-II). San Antonio, TX: Pearson.

Wilkinson, G. S., \& Robertson, G. J. (2006). Wide Range Achievement Test 4 professional manual. Lutz, FL: Psychological Assessment Resources.

Williams, J. B. (1988). A structured interview guide for the Hamilton Depression Rating Scale. Archives of General Psychiatry, 45, 742-747. doi:

10.1001/archpsyc.1988.01800320058007 
Table 1

Demographic information for entire sample $(N=62)$

\begin{tabular}{|c|c|}
\hline & $M(S D)$ or $N(\%)$ \\
\hline Age & $36.32(10.45)$ \\
\hline \multicolumn{2}{|l|}{ Gender } \\
\hline Female & $53(85.5 \%)$ \\
\hline Male & $9(14.5 \%)$ \\
\hline \multicolumn{2}{|l|}{ Ethnicity } \\
\hline African American & $4(6.5 \%)$ \\
\hline Caucasian & $58(93.5 \%)$ \\
\hline \multicolumn{2}{|l|}{ Marital status } \\
\hline Married & $28(45.2 \%)$ \\
\hline Not married & $34(54.8 \%)$ \\
\hline \multicolumn{2}{|l|}{ Employment status } \\
\hline Full-time & $25(40.3 \%)$ \\
\hline Part-time & $6(9.7 \%)$ \\
\hline Unemployed & $26(41.9 \%)$ \\
\hline Retired & $1(1.6 \%)$ \\
\hline Receiving disability & $4(6.5 \%)$ \\
\hline \multicolumn{2}{|l|}{ Household income } \\
\hline Less than $\$ 60,000 /$ year & $43(69.5 \%)$ \\
\hline Greater than $\$ 60,000 /$ year & $19(30.6 \%)$ \\
\hline \multicolumn{2}{|l|}{ Level of education } \\
\hline Grade school & $1(1.6 \%)$ \\
\hline High school & $26(41.9 \%)$ \\
\hline College & $23(37.1 \%)$ \\
\hline Graduate school & $12(19.4 \%)$ \\
\hline IQ & $97.43(18.08)$ \\
\hline CAPI physical abuse risk & $124.20(88.90)$ \\
\hline Parent trauma & $32(51.6 \%)$ \\
\hline
\end{tabular}


Table 2

Demographic variables broken down by faking and non-faking groups

\begin{tabular}{|c|c|c|}
\hline & Faking $^{\mathrm{a}}$ & Non-faking ${ }^{\mathrm{b}}$ \\
\hline & $M(S D)$ or $\%$ & $M(S D)$ or $\%$ \\
\hline Age & $36.54(11.13)$ & $36.20(10.20)$ \\
\hline \multicolumn{3}{|l|}{ Gender } \\
\hline Female & $21(95.5 \%)$ & $32(80.0 \%)$ \\
\hline Male & $1(4.5 \%)$ & $8(20.0 \%)$ \\
\hline \multicolumn{3}{|l|}{ Ethnicity } \\
\hline African American & $1(4.5 \%)$ & $3(7.5 \%)$ \\
\hline Caucasian & $21(95.5 \%)$ & $27(92.5 \%)$ \\
\hline \multicolumn{3}{|l|}{ Marital status } \\
\hline Married & $12(54.5 \%)$ & $16(40.0 \%)$ \\
\hline Not married & $10(45.5 \%)$ & $24(60.0 \%)$ \\
\hline \multicolumn{3}{|l|}{ Employment status } \\
\hline Full-time & $12(54.5 \%)$ & $13(32.5 \%)$ \\
\hline Part-time & $1(4.5 \%)$ & $5(12.5 \%)$ \\
\hline Unemployed & $8(36.4 \%)$ & $18(45.0 \%)$ \\
\hline Retired & 0 & $1(2.5 \%)$ \\
\hline Receiving disability & $1(4.5 \%)$ & $3(7.5 \%)$ \\
\hline \multicolumn{3}{|l|}{ Household income } \\
\hline Less than $\$ 60,000 /$ year & $14(63.6 \%)$ & $29(72.5 \%)$ \\
\hline Greater than $\$ 60,000 /$ year & $8(36.4 \%)$ & $11(27.5 \%)$ \\
\hline \multicolumn{3}{|l|}{ Level of education } \\
\hline Grade school & $1(4.5 \%)$ & 0 \\
\hline High school & $10(45.5 \%)$ & $16(40.0 \%)$ \\
\hline College & $8(36.4 \%)$ & $15(37.5 \%)$ \\
\hline Graduate school & $3(13.6 \%)$ & $9(22.5 \%)$ \\
\hline CAPI physical abuse risk & $109.95(101.41)$ & $132.05(81.53)$ \\
\hline
\end{tabular}


Parent trauma

$10(45.5 \%)$

$22(55.5 \%)$

Note. ${ }^{\mathrm{a}} n=22 .{ }^{\mathrm{b}} n=40$. 


\section{Table 3}

BeanFest game and test phase variables using the simplified version

\section{Game Phase}

B1 positive approach

$.79(.17)$

B2 positive approach

$.86(.15)$

B3 positive approach

$.90(.15)$

B1 negative approach

$.31(.28)$

B2 negative approach

$.18(.28)$

B3 negative approach

.15 (.26)

$\mathrm{B} 1$ positive accurate

$.79(.17)$

B2 positive accurate

$.86(.15)$

B3 positive accurate

$.89(.15)$

B1 negative accurate

$.67(.28)$

B2 negative accurate

$.80(.28)$

B3 negative accurate

$.82(.27)$

Number of games won

6.94 (3.66)

Number of games lost

$.22(.64)$

Test Phase

Learning bias

$.04(.19)$

Attitude generalization

$.02(.29)$

Weighting bias

$-.01(.32)$

Note. $\mathrm{B} 1=$ Block $1, \mathrm{~B} 2=$ Block $2, \mathrm{~B} 3=$ Block 3 
Table 4

Correlational matrix of BeanFest variables and participant demographic information

\begin{tabular}{|c|c|c|c|c|c|c|c|c|c|c|}
\hline & Age & WASI-II & BDI-II & $\begin{array}{c}\text { Pos. } \\
\text { approach }\end{array}$ & $\begin{array}{c}\text { Neg. } \\
\text { approach }\end{array}$ & $\begin{array}{c}\text { Pos. } \\
\text { accurate }\end{array}$ & $\begin{array}{c}\text { Neg. } \\
\text { accurate }\end{array}$ & $\begin{array}{c}\text { Learning } \\
\text { bias }\end{array}$ & Weighting & $\begin{array}{l}\text { Attitude } \\
\text { gen. }\end{array}$ \\
\hline Age & -- & & & & & & & & & \\
\hline WASI-II & .007 & -- & & & & & & & & \\
\hline BDI-II & .23 & $-.33 * *$ & -- & & & & & & & \\
\hline Pos. approach & -.09 & $.27 * *$ & .006 & -- & & & & & & \\
\hline Neg. approach & .10 & $-.42 * *$ & .15 & $-.47 * *$ & -- & & & & & \\
\hline Pos. accurate & -.10 & $.27 *$ & .007 & $.99 * *$ & $-.47 * *$ & -- & & & & \\
\hline Neg. accurate & -.10 & $.41^{* *}$ & .15 & $.47^{* *}$ & $-1.0^{* *}$ & $.47^{* *}$ & -- & & & \\
\hline Learning bias & .02 & -.21 & -.10 & .10 & $.47 * *$ & .11 & $-.47 * *$ & -- & & \\
\hline Weighting & -.07 & -.03 & .01 & .17 & .21 & .17 & -.21 & $.46^{* *}$ & -- & \\
\hline Attitude gen. & -.03 & -.01 & -.06 & .21 & $.26^{*}$ & .21 & $-.26^{*}$ & $.64 * *$ & $.92 * *$ & -- \\
\hline
\end{tabular}

Note. Approach and accuracy scores (by valence) were aggregated across Blocks 1-3 on BeanFest. Refer to Table 3 for positive and negative approach and accuracy scores by valence. ${ }^{*} p<.01, * p<.05$. 
Table 5

Comparison of faking and non-faking participants across main outcome variables

\begin{tabular}{|c|c|c|c|c|c|c|c|}
\hline & Faking $^{\mathrm{a}}$ & Non-faking ${ }^{b}$ & & & & & \\
\hline & $M(S D)$ or $\%$ & $M(S D)$ or $\%$ & $t$ & $\chi^{2}$ & $p$ & Effect size & Interpretation \\
\hline \multicolumn{8}{|l|}{ Primary outcomes } \\
\hline CPS-involved & $45.5 \%$ & $55.5 \%$ & & .51 & .47 & .09 & No effect \\
\hline IQ & $93.45(16.10)$ & $99.62(18.92)$ & -1.29 & & .20 & .02 & Small effect \\
\hline Reading comp. & $98.90(23.22)$ & $101.12(17.42)$ & -.42 & & .66 & .002 & No effect \\
\hline LSRP P1 & $24.50(6.08)$ & $24.55(6.52)$ & -.03 & & .97 & .00 & No effect \\
\hline LSRP P2 & $19.31(5.70)$ & $22.50(7.86)$ & -1.67 & & .10 & .04 & Small effect \\
\hline LSRP total & $43.81(10.82)$ & $47.05(13.03)$ & -.98 & & .32 & .01 & Small effect \\
\hline Learning bias & $.14(.19)$ & $-.007(.17)$ & 2.95 & & .005 & $.13 * *$ & Large effect \\
\hline Attitude gen. & $.15(.30)$ & $-.03(.27)$ & 2.47 & & .02 & $.09^{*}$ & $\begin{array}{c}\text { Moderate } \\
\text { effect }\end{array}$ \\
\hline Weighting bias & $.14(.34)$ & $-.08(.28)$ & 2.67 & & .01 & $.11^{*}$ & $\begin{array}{c}\text { Moderate } \\
\text { effect }\end{array}$ \\
\hline \multicolumn{8}{|c|}{ Exploratory outcomes } \\
\hline MCSDS & $24.28(4.58)$ & $17.20(5.43)$ & 5.06 & & .00 & .30 & Large effect \\
\hline BDI-II & $10.09(11.43)$ & $10.57(7.93)$ & -.19 & & .84 & .00 & No effect \\
\hline Parent trauma & $45.5 \%$ & $55.5 \%$ & & .51 & .47 & .09 & No effect \\
\hline
\end{tabular}




\begin{tabular}{|c|c|c|c|c|c|c|}
\hline Child learning & $9.5 \%$ & $52.5 \%$ & 10.82 & .001 & .42 & $\begin{array}{c}\text { Moderate } \\
\text { effect }\end{array}$ \\
\hline Child behavior & $52.4 \%$ & $63.2 \%$ & .65 & .42 & .10 & Small effect \\
\hline
\end{tabular}

Note. LSRP Primary Psychopathy, Secondary Psychopathy, and Total Score were derived from a version of the LSRP utilizing a 5-point Likert scale (instead of a 4-point Likert scale which is typically used). Therefore, these means cannot be directly compared to other studies using the 4point Likert scale version of the LSRP. Eta squared was used to determine the effect size of the independent-samples $t$-tests. According to Cohen (1988), $.01=$ small effect, $.06=$ moderate effect, and $.14=$ large effect. Cramer's V was used to determine the effect size of the chi-square analyses; it is measured from .0-1.0, and scores below 0.10 are considered to have no effect (Field, 2009). $* * p<.01, * p<.05$

${ }^{\mathrm{a}} n=22 .{ }^{\mathrm{b}} n=40$. 
Table 6

Correlational matrix including primary and exploratory outcome variables

\begin{tabular}{|c|c|c|c|c|c|c|c|c|c|c|c|c|c|c|c|}
\hline & $\begin{array}{c}\text { CAPI } \\
\text { Lie }\end{array}$ & TX & LB & WB & $\mathrm{AG}$ & M-C & WASI & WIAT & LSRP1 & LSRP2 & LSRPT & $\begin{array}{c}\text { BDI- } \\
\text { II }\end{array}$ & TR & $\begin{array}{l}\text { Ch. } \\
\text { learn }\end{array}$ & $\begin{array}{l}\text { Ch. } \\
\text { beh }\end{array}$ \\
\hline CAPI L & -- & & & & & & & & & & & & & & \\
\hline TX & -.10 & -- & & & & & & & & & & & & & \\
\hline LB & $.28^{*}$ & -.007 & -- & & & & & & & & & & & & \\
\hline WB & $.26^{*}$ & .15 & $.48 * *$ & -- & & & & & & & & & & & \\
\hline $\mathrm{AG}$ & .24 & .15 & $.64 * *$ & $.93 * *$ & -- & & & & & & & & & & \\
\hline M-C & $.73 * *$ & -.05 & .20 & .12 & .14 & -- & & & & & & & & & \\
\hline WASI & $-.42 * *$ & $.34 * *$ & -.21 & -.02 & -.01 & $-.29 *$ & -- & & & & & & & & \\
\hline WIAT & $-.35 * *$ & $.51 * *$ & -.14 & -.05 & -.08 & -.25 & $.73 * *$ & -- & & & & & & & \\
\hline LSRP1 & .08 & $-.29 *$ & -.08 & -.11 & -.11 & -.04 & $-.44 * *$ & $-.46^{* *}$ & -- & & & & & & \\
\hline LSRP2 & -.06 & -.24 & -.20 & -.24 & -.22 & -.16 & $-.39 * *$ & $-.41 * *$ & $.63 * *$ & -- & & & & & \\
\hline LSRPT & .008 & $-.29 *$ & -.16 & -.20 & -.19 & -.11 & $-.46 * *$ & $-.48 * *$ & $.88 * *$ & $.91 * *$ & -- & & & & \\
\hline BDI-II & -.06 & -.10 & -.10 & .02 & -.06 & $-.29 *$ & $-.33 * *$ & $-.28 *$ & .07 & $.30^{*}$ & .21 & -- & & & \\
\hline TR & .06 & .22 & -.10 & .02 & .03 & .10 & .22 & .14 & .03 & -.13 & -.05 & -.24 & -- & & \\
\hline Ch. learn & .23 & -.004 & .22 & .23 & .24 & .18 & .006 & -.08 & -.09 & $-.28 *$ & -.21 & -.04 & .22 & -- & \\
\hline Ch. beh & .14 & -.09 & .08 & -.09 & -.03 & .05 & -.006 & .01 & -.02 & -.08 & -.06 & -.004 & .05 & .25 & -- \\
\hline
\end{tabular}


Table 7

Stepwise regression predicting CAPI Lie Scale from significant predictors

\begin{tabular}{lccc}
\hline & $B$ & $S E B$ & $\beta$ \\
\cline { 2 - 4 } Prediction model & -1.94 & 1.05 & $.73^{* *}$ \\
Constant & .39 & .05 & \\
MCSDS score & & & \\
Excluded variables & -.19 & & \\
WASI-II score & -.11 & \\
WIAT-III score & .15 & \\
Learning bias & .16 & \\
Weighting bias &
\end{tabular}

Note. $R^{2}=.54$, Adjusted $R^{2}=.53$ for prediction model. ${ }^{* *} p=.000$. 
Table 8

Stepwise regression predicting CAPI Lie Scale from primary outcomes

\begin{tabular}{lccc}
\hline & $B$ & $S E B$ & $\beta$ \\
\cline { 2 - 4 } Prediction model & 13.40 & 2.45 & \\
Constant & -.07 & .02 & $-.39^{*}$ \\
WASI-II score & .05 & & \\
Excluded variables & -.03 & \\
Treatment setting & -.23 & \\
WIAT-III score & .21 & \\
LSRP primary & & \\
Learning bias &
\end{tabular}

Note. $R^{2}=.15$, Adjusted $R^{2}=.13$ for prediction model. $* p=.003$. 


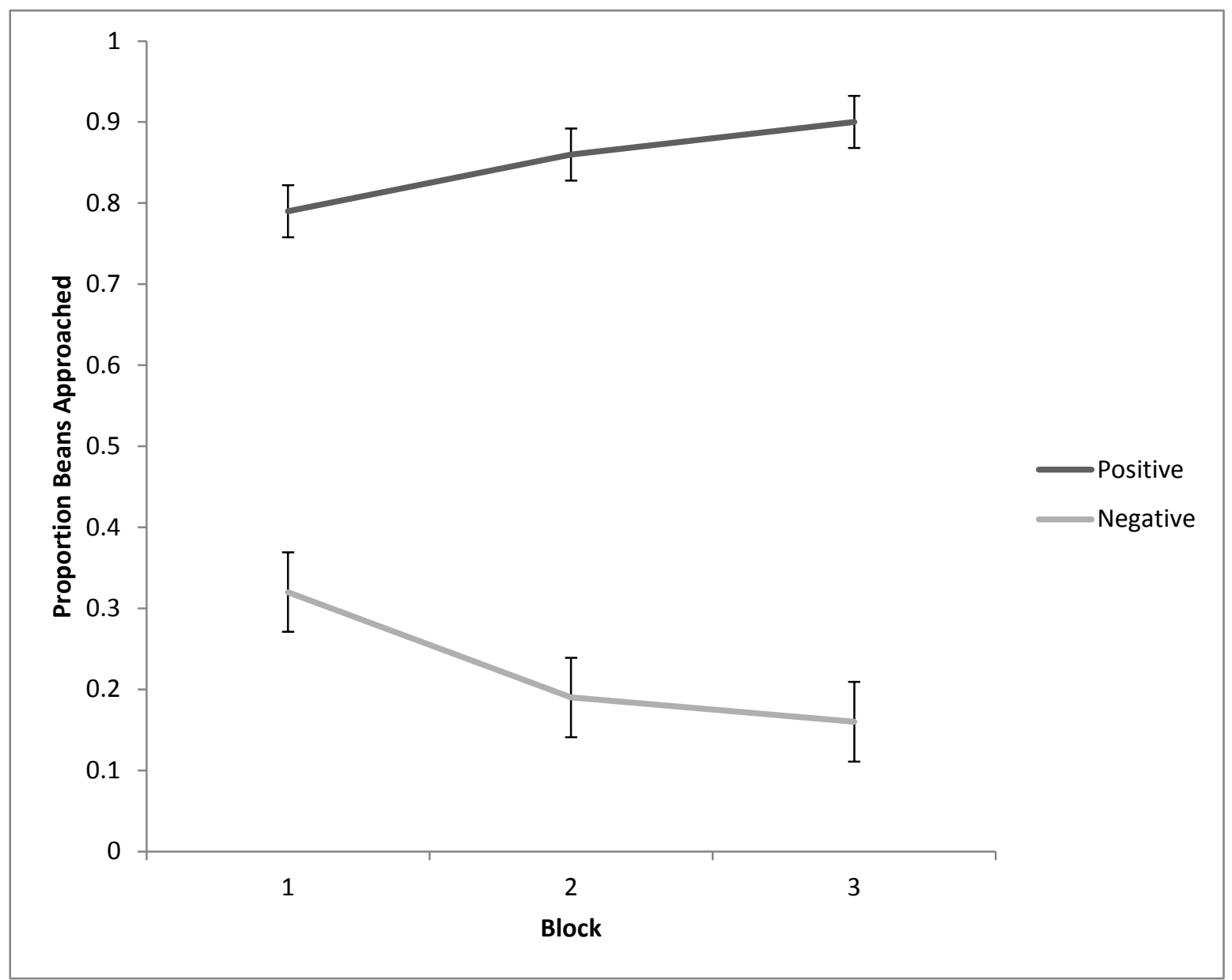

Figure 1. Proportion of positive and negative beans approached across Blocks 1-3 in the BeanFest game phase. Error bars represent the standard error of the mean. 


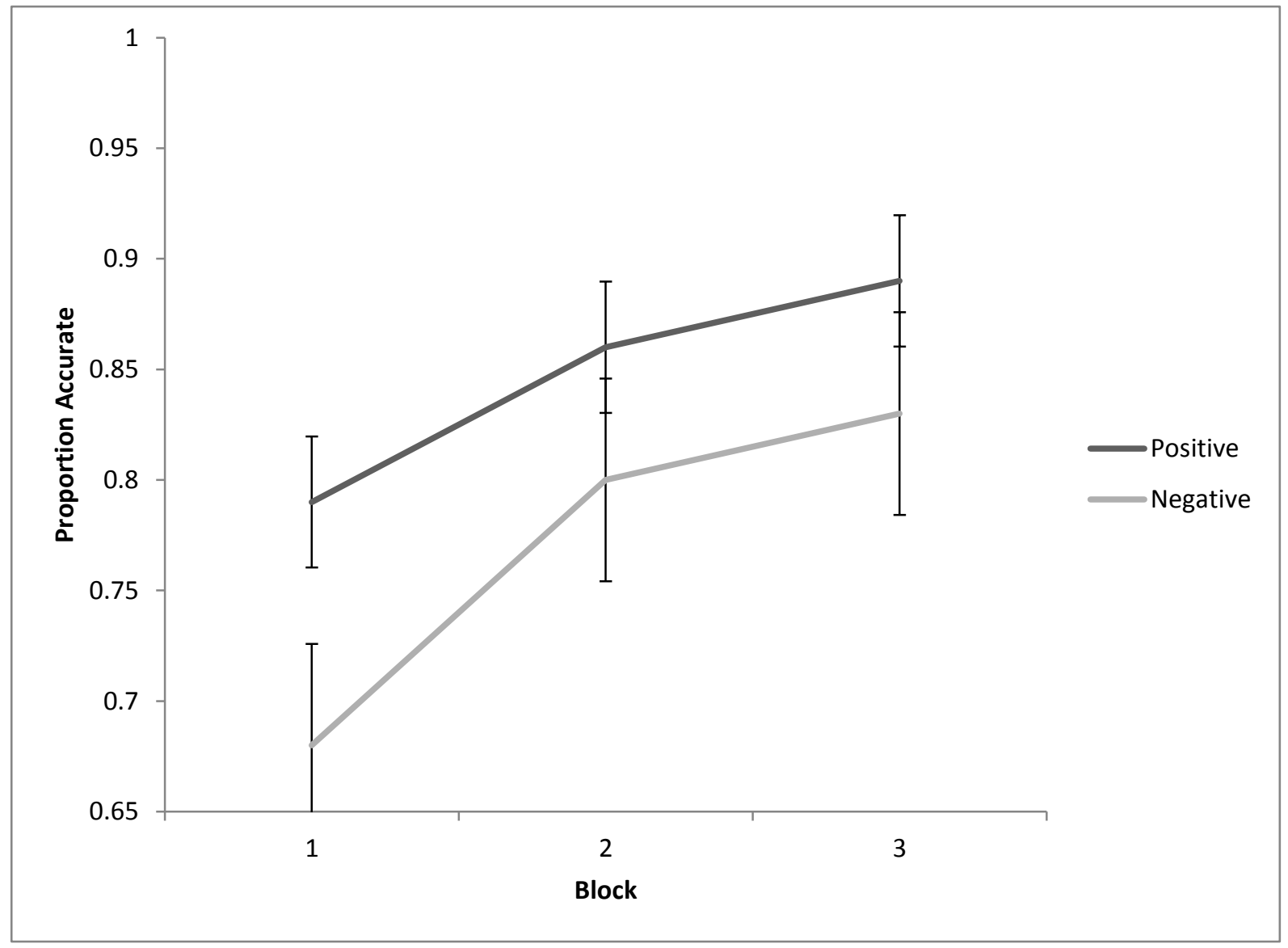

Figure 2. Proportion of positive and negative beans accurately chosen or avoided across Blocks 1-3 in the BeanFest game phase. Error bars represent the standard error of the mean. 
Appendix A - Sample Permission to Contact Flyer

\section{Are you interested participating in a study about parenting practices and attitudes?}

Researchers from the Psychology Department at West Virginia University are currently seeking parents for a research study examining parenting practices and attitudes. This study is being done to fulfill the requirements of a Doctoral degree.

To participate in the study you or your child (17 and under) must be currently receiving services from a participating agency.

The study will take place in your home and will involve:

- Filling out some questionnaires

- About 1.5 hours of your time; assessment times are flexible

For more information, please call 304-293-1677 and leave a message for Amanda Costello.

Email: Amanda.Costello@mail.wvu.edu

Participants will receive a $\$ 30.00$ gift card to Wal-Mart.

\section{Interested in helping researchers learn more about parenting attitudes?}

I agree to allow the Department of Psychology at West Virginia University to contact me by telephone regarding my interest in the research study 'Studying practices and attitudes of parents in community mental health agencies.'

Full Name

Signature

Phone Number 
Appendix B - Presentation of Measures

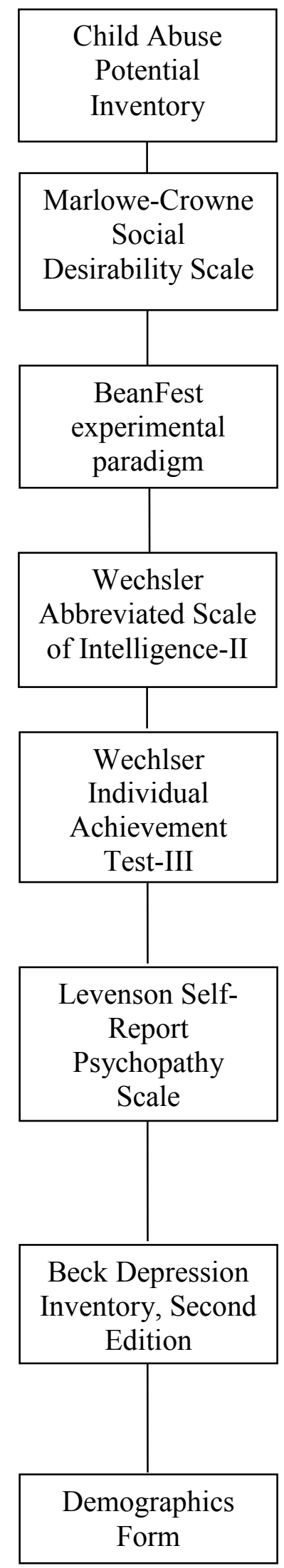


Appendix C - Instructions for BeanFest (Fazio et al., 2004)

\section{INSTRUCTIONS FOR BEANFEST}

You are going to play a computer game that we call BEANFEST. The game involves beans and points. The objective of the game is to increase your points by selecting beans that increase your point total and avoiding beans that decrease your point total. You will begin the game with 50 points and will be presented with a series of beans, one bean at a time. Your job is to select the "good" beans and avoid the "bad" beans. For every "good" bean you select, your point total will increase by 10 points until you reach 100, at which point you win the game. However, for every "bad" bean you select, you will lose 10 points, until your score reaches 0 , at which point you will lose the game. As you play the game, it is important to learn which beans have a positive value and choose them in order to gain points. Also, you need to learn which beans have a negative value so that you can avoid them in order to avoid losing points.

The beans look different in two important ways. First, they are different in shape circular to oval to oblong. Second, they are different in the extent to which they are speckled marked with few to some to many dots. It is important to learn how to tell the beans apart in order to be successful. Here are a few examples.

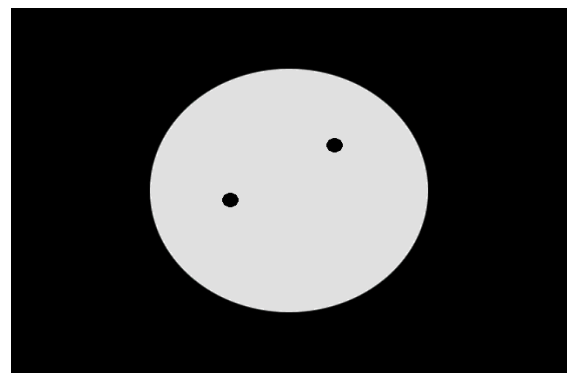

Circular with few speckles

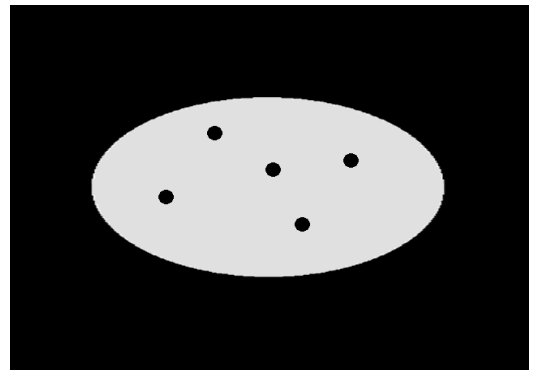

Oval with some speckles

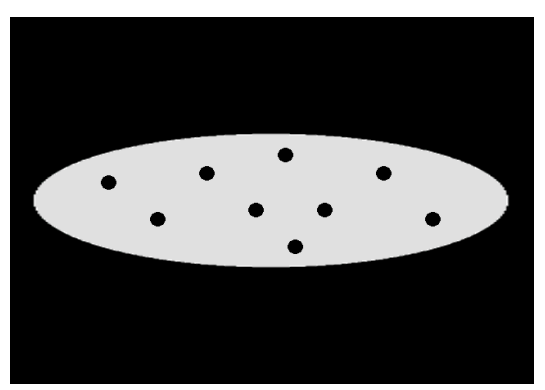

Oblong with many speckles

BEANFEST consists of three rounds, and there are many trials in each round. On each trial, you will be presented with a bean in the upper portion of the screen. Use the keyboard to indicate whether you wish to select the bean or not. Press the " $\mathrm{K}$ " key, if you wish to select the bean. Press the "D" key, if you do not want to select the bean.

The lower third of the screen provides you with valuable information. To the left is your point meter. It displays your current points as a bar ranging from 0 to 100 . Your specific point level is displayed numerically. In addition, the point bar fluctuates to reflect your current level. 
If you choose not to select the presented bean, your decision will be shown in the lower right of the screen, as below. Your point value will not change.

\section{Decision NO \\ Effect bean \\ would have had 10}

If you select the bean, your decision will be shown in the lower right of the screen, as below, and your point value will be updated. The effect of the bean will also be displayed, with negative numbers indicating that the bean reduced your points and positive numbers indicating that the bean increased your points. So, for example, if you choose a bean whose value is +10 , your point value will increase by 10 and your display will show the following:

$\begin{array}{ll}\text { Decision } & \text { YES } \\ \text { Effect of bean } & 10\end{array}$

If, on the other hand, you choose a bean whose value is -10 , your point value will decrease by 10 and your display will show the following:

$\begin{array}{ll}\text { Decision } & \text { YES } \\ \text { Effect of bean } & -10\end{array}$

As noted earlier, you will begin the game with 50 points. You should try to do your best throughout the game to gain points and avoid losing points by making good decisions about which beans to select. Reaching 100 represents winning the game, and reaching 0 represents losing the game. If your point level ever reaches 100 , you will immediately be notified of the fact that you have won. You will then start a new game with 50 points. If your point level ever reaches 0 , you will immediately be notified of the fact that you have lost. Again, you will then start a new game with 50 points. In any new games, the beans retain their original values. That is, previously good beans continue to increase your points and previously bad beans continue to decrease your points.

The game actually begins with a short practice block of 4 trials. These 4 beans are just a few of the ones that you will see during the game, but they are of the same type and have the same value as they will during the game. After you have begun the game I can't answer any more questions. So, this is your first opportunity to begin to learn about some of the beans. For these 4 practice trials, always respond YES. The practice trials are intended as an opportunity to familiarize you with the game and feedback displays.

Once the practice block is over, there will be a break in case you have any questions about the game. At that time, we can address them before starting the game. When you start the actual game, you will begin with 50 points. Remember, try to increase your points and avoid losing your points. Press the "K" key if you wish to select the bean, and the " $\mathrm{D}$ " key if you do not. At the beginning of the game, you will probably select beans somewhat indiscriminately, simply because you do not know any better. You will need to learn which beans have positive values and which have negative values. As you begin to do so, you can be more selective about which beans to choose and which to avoid, which is the only way to increase your points. Remember, the beans vary visually in two (and only) two important ways: (1) shape, from 
circular to oval to oblong and (2) number of speckles, from few to some to many. Try your best on the task.

\section{TEST PHASE}

In this final test phase, you will be presented with the beans to which you were just exposed. No point meter or feedback will be displayed. In this part of the experiment, we simply want to know whether you believe a given bean to be "good" or "bad." Again, use the keyboard. Press the " $\mathrm{K}$ " key if this is a bean that you would select, i.e., one that you believe increases your point level. Press the "D" key if this is a bean that you would not select, i.e., one that you believe would decrease your points. Try to respond as accurately and as quickly as possible. Don't be in such a hurry that you regret your response. But, try to respond as quickly as you can without sacrificing accuracy. So, maximize both the speed and the accuracy of your responses. There may be some beans that are unfamiliar or that you are unsure about. For these beans make your best guess. Just be sure to respond on each and every trial within the allotted 5 seconds.

There is no practice block. So be ready. 
Appendix D - Demographics Form

Participant ID \#:

Demographic/ Background Form

Your Date of Birth:

Your Ethnicity:

American Indian/ Alaskan Native Asian

Native Hawaiian/ Other Pacific Islander specify):

Hispanic/ Latino American
Your Age:

Black/ African American

White/Caucasian

Other (please

Your Marital status (Check One)

Single:

Married:
Divorced:

Widowed:
Other (please specify):

Your Highest grade level of education completed (Circle One)

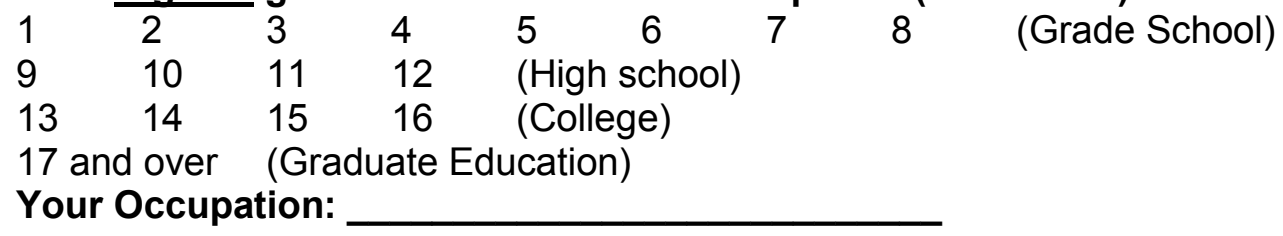

Employment Status (Check One)

Employed Full-time:

Employed Part-time:

Retired:

Unemployed:

Foster-parent Full-time:

Receiving disability:

Annual Household Income (Check one)

Less than 20,000

$20,000-40,000$

$60,000-80,000$

$80,000-100,000$

$40,000-60,000$

More than 100,000

Have you ever experienced abuse/trauma? Y/N

If yes, please describe the nature of the abuse/trauma:

Number of Child Protective Services reports for self:

Year(s):

Nature of report:

Outcome:

Number of Child Protective Services reports for your child/children:

Year(s):

Nature of report:

Outcome:

Do you have a child who has learning problems?

If yes, please describe:

Do you have a child who has behavioral problems?

If yes, please describe: 